\title{
COSMOLOGICAL TIME VERSUS CMC TIME IN SPACETIMES OF CONSTANT CURVATURE*
}

\author{
LARS ANDERSSON ${ }^{\dagger}$, THIERRY BARBOT ${ }^{\ddagger}$, FRANÇOIS BÉGUIN $^{\S}$, AND \\ ABDELGHANI ZEGHIB
}

\begin{abstract}
In this paper, we investigate the existence of foliations by constant mean curvature (CMC) hypersurfaces in maximal, globally hyperbolic, spatially compact, spacetimes of constant curvature.

In the non-positive curvature case (i.e. for flat and locally anti-de Sitter spacetimes), we prove the existence of a global foliation of the spacetime by CMC Cauchy hypersurfaces. The positive curvature case (i.e. locally de Sitter spacetimes) is more delicate: in general, we are only able to prove the existence of a foliation by CMC Cauchy hypersurfaces in a neighbourhood of the past (or future) singularity.

Except in some exceptional and elementary cases, the leaves of the foliation we construct are the level sets of a time function, and the mean curvature of the leaves increases with time. In this case, we say that the spacetime admits a $C M C$ time function.

Our proof is based on using the level sets of the cosmological time function as barriers. A major part of the work consists of proving the required curvature estimates for these level sets. One of the difficulties is the fact that the local behaviour of the cosmological time function near one point depends on the global geometry of the spacetime.
\end{abstract}

Key words. Spacetimes, constant curvature, time function, cosmological time, CMC.

AMS subject classifications. 53C50, 53C42, 53C80, 83C20.

1. Introduction. The purpose of the present paper is to study the existence of foliations by CMC Cauchy hypersurfaces, and the existence of CMC time functions on maximal globally hyperbolic, spatially compact, spacetimes of constant curvature.

1.1. CMC time functions as canonical time functions. Spacetimes considered as cosmological models in generel relativity are usually assumed to be globally hyperbolic. By a classical theorem of Geroch, every such spacetime $(M, g)$ admits a time function, that is a function $t: M \rightarrow \mathbb{R}$ which is strictly increasing along every future-oriented causal curve. This time function is by no means unique : the set of all time functions on a given spacetime contains open sets of functions for the $C^{1}$ Whitney topology. Nevertheless, on some spacetimes, it is possible to find a canonical time function. By "canonical", we mean "defined in a coordinate invariant manner".

The primary interest of a canonical time function is of course to provide a good notion of time. Introducing a canonical time function also allows one to describe the spacetime as a one parameter family of Riemannian spaces (the level sets of the function) indexed by time. As a consequence, it is possible to measure the geometric distortion of the spacetime (how different is the spacetime from a metric product

\footnotetext{
*Received July 03, 2009; accepted for publication February 24, 2011.

$\dagger$ Albert Einstein Institute, Am Mühlenberg 1, D-14476 Potsdam, Germany \& Department of Mathematics, University of Miami, Coral Gables, FL 33124, USA (laan@aei.mpg.de). Supported in part by the NSF, under contract no. DMS 0104402 with the University of Miami.

‡CNRS, UMPA, École Normale Supérieure de Lyon (Thierry.Barbot@univ-avignon.fr). Supported in part by ACI "Structures géométriques et Trous Noirs".

$\S$ Mathématiques, Université Paris Sud. (Francois.Beguin@math.u-psud.fr). Supported in part by ACI "Structures géométriques et Trous Noirs".

`CNRS, UMPA, École Normale Supérieure de Lyon (Abdelghani.Zeghib@ens-lyon.fr). Supported in part by ACI "Structures géométriques et Trous Noirs".
} 
$\left.\left(I,-d t^{2}\right) \times(N, h) ?\right)$, or to describe the asymptotic geometric behaviour of the spacetime as one approaches the initial (or future) singularity (or when times goes to infinity).

One example of canonical time function is the so-called cosmological time. Given a spacetime $(M, g)$, one may consider the function $\tau: M \rightarrow[0,+\infty]$ defined as follows: $\tau(x)$ is the supremum of the lengths of all past-directed timelike curves starting at $x$. If $\tau(x)$ is finite for every $x$ in $M$ and depends continuously on $x$ (which is the case e.g. in the classical Robertson-Walker models), then $\tau$ is a time function on $M$ : the cosmological time of $M$. The main drawback of the cosmological time is that it typically has a poor regularity $\left(C^{1}\right.$ but not $\left.C^{2}\right)$.

In this paper, we will be considering another type of canonical time functions. A CMC time function of a spacetime $(M, g)$ is a time function $t: M \rightarrow I \subset \mathbb{R}$ such that for every $a \in I$, the level set $t^{-1}(a)$ is a Cauchy hypersurface with constant mean curvature equal to $a$. If such a function exists, the spacetime is foliated by Cauchy hypersurfaces with constant mean curvature, and the mean curvature of these Cauchy hypersurfaces increases with time. A simple consequence of the maximum principle is that a CMC time function with compact level sets, when it exists, is automatically unique. Hence, CMC time functions are examples of canonical time functions.

In addition to being canonical time functions, CMC time functions are interesting because of the important role they play in the study of the Einstein equations. The study of the global properties of spacetimes solving the Einstein equations plays a central role both in differential geometry and General Relativity. However, with the exception of results which rely on small data assumptions (nonlinear stability results) or the assumption of symmetries, many fundamental questions about the global structure of Einstein spacetimes remain open, including cosmic censorship, structure of singularities, and existence of global foliations by Cauchy hypersurfaces with controlled geometry. Recall that the Einstein equations are hyperbolic only in a weak sense, and therefore in order to approach its Cauchy problem from a PDE point of view, it is necessary either to impose gauge conditions, or to extract a hyperbolic system by modifying the equation. The constant mean curvature (CMC) condition is an important gauge condition in the study of the Cauchy problem of the Einstein equation, and hence in General Relativity. The CMC time gauge is known to lead to a well-posed Cauchy problem in conjunction with the zero shift condition [27] as well as with the spatial harmonic gauge condition [8]. In the Hamiltonian formulation of the Einstein equations, the volume of a CMC hypersurface can be viewed as the canonical dual to the CMC time, see [30]. In the case of $2+1$ dimensional spacetimes, this point of view leads to a formulation of the Einstein equations in CMC gauge as a time-dependent finite dimensional Hamiltonian system on the cotangent bundle of Teichmüller space (see [41]).

There are numerous results concerning the existence of global foliations by CMC hypersurfaces and CMC time functions under various symmetry conditions, for spacetimes with and without matter. See $[2,44]$ for recent surveys. It should be noted that examples of Ricci flat spacetimes which do not contain any CMC Cauchy hypersurface were recently constructed [28]. However, it is not yet known if these examples are stable.

1.2. Maximal globally hyperbolic spatially compact spacetimes of constant curvature. Recall that a Lorentz manifold, or spacetime, $(M, g)$ is globally hyperbolic if it contains a Cauchy hypersurface $S$, i.e. a spacelike hypersurface such that each inextendible causal curve in $M$ intersects $S$ at exactly one point. A glob- 
ally hyperbolic spacetime is maximal if it cannot be extended in the class of globally hyperbolic spacetimes. For brevity we use the acronym MGHC for maximal globally hyperbolic spacetimes whose Cauchy hypersurfaces are compact. An equivalent definition is that a spacetime $M$ is MGHC if and only if it admits a time function $t: M \rightarrow \mathbb{R}$ which is proper (i.e. $t^{-1}([a, b])$ is a compact subset of $M$ for every compact interval $[a, b] \subset \mathbb{R})$.

Several authors, see eg. [16], use the term cosmological spacetime to denote a MGHC spacetime satisfying the timelike convergence, or strong energy condition, i.e. $\operatorname{Ric}(v, v) \geq 0$ for every timelike vector $v$ (see below).

Spacetimes with constant (sectional) curvature are special cases of spacetimes satisfying the vacuum Einstein equations with cosmological constant. They constitute an important subclass of spacetimes, where one may expect to understand the fundamental questions, including the cosmic censorship problem completely. However, even within this subclass, there are still open questions relating to the existence and properties of constant mean curvature foliations, and the asymptotic structure at cosmological singularities is not fully understood.

MGHC spacetimes with constant curvature have a locally trivial geometry, being locally isometric to Minkowski space, de Sitter space or anti-de Sitter space and thus the partial differential equations aspect of the analysis of these spacetimes is trivial. However, the topology of these spacetimes may be highly nontrivial and although the spacetimes under consideration have a local isometry pseudo-group of maximal dimension, they typically have trivial (global) isometry groups. The interplay between the topology and the causal structure of the spacetime will be the source of most of the difficulties encountered in our work.

Having a locally trivial geometry, a spacetime with constant curvature cannot be considered as an accurate model of the Universe at small scales (e.g. since the Weyl tensor of such a spacetime vanishes, it cannot model phenomena like gravitationnal waves). Nevertheless, we do think that the study of such spacetimes is pertinent. Indeed, considering spacetimes with constant curvature allows one to ignore the local issues, in order to focus on the global topological and geometrical problems. Moreover, recall that very little is known on the global geometry of the solutions of the Einstein equations except for results which rely on small data assumptions (nonlinear stability results) or the assumption of symmetries. The spacetimes we consider do not have global symmetries, and do not correspond to the usual "small data situation", so the situation we are handling leads into unexplored areas.

MGHC spacetimes with constant curvature can also be considered as large scale approximation of more realistic spacetimes. From this point of view, our result could be thought as starting points for studies of non-homogenous deformations of MGHC spacetimes with constant curvature .

Among the spacetimes we consider here are those with positive constant curvature, i.e. MGHC de Sitter spacetimes. An essential feature of the current standard model in cosmology is the accelerated expansion of the universe. In order to achieve accelerated expansion, the strong energy condition must be violated, which leads one to consider spacetimes with positive cosmological constant, i.e. spacetimes of de Sitter type. The timelike convergence condition is violated in these spacetimes and hence the standard proof of uniqueness of CMC foliations does not apply. Nevertheless, we shall show the existence of a large class of MGHC de Sitter spacetimes which admit a CMC time function, and thus a unique $\mathrm{CMC}$ foliation. 
On the other hand, spacetimes of anti-de Sitter type play an important role in the AdS/CFT correspondence, which is currently being intensely investigated by string theorists.

The systematic study of spacetimes of constant sectional curvature was initiated by Mess [40], following work by among others Margulis [39] and Fried [31]. The classification of maximal globally hyperbolic flat spacetimes with complete Cauchy hypersurfaces has recently been completed by Barbot [11], following work of Bonsante $[23,24]$ and others. This classification in the de Sitter case has been performed by Scannell in his thesis [45]. The classification in the anti-de Sitter case is not yet fully understood, but what is known is enough for our purpose - and it appears that this case is in some way the simplest one: we will prove that every anti-de Sitter MGHC spacetime admits a CMC time function, with no further restriction. However, it remains a difficult case, due to the necessicity to grasp the geometrical feature of the anti-de Sitter space, which will be developed in this paper.

1.3. Conventions and definitions. Consider a $n$-dimensional spacetime $M$. Let $S \subset M$ be a spacelike hypersurface, and $\nu$ be its future directed unit normal. Then for $X, Y$ tangent to $S$, the second fundamental form is given by $\operatorname{II}(X, Y)=$ $\left\langle\nu, \nabla_{X} Y\right\rangle=-\left\langle\nabla_{X} \nu, Y\right\rangle$. The mean curvature of $S$ is defined by $H_{S}=\operatorname{tr}(\mathrm{II}) /(n-1)$. The hypersurface $S$ is called a $C M C$ hypersurface if $H_{S}$ is constant over $S$.

If $M$ satisfies the timelike convergence condition (i.e. if $\operatorname{Ric}(V, V) \geq 0$ for timelike vectors $V$ ) and has compact Cauchy hypersurfaces, then for each $p \in M$ and for each $\tau \neq 0$, there is at most one compact CMC surface containing $x$ with mean curvature $\tau$. The timelike convergence condition holds in flat and locally anti-de Sitter spacetimes, but fails in locally de Sitter spacetimes.

A CMC foliation of a spacetime $M$ is a foliation whose leaves are CMC hypersurfaces.

REMARK 1.1. Let $\mathcal{F}$ be a CMC foliation of $M$ whose leaves are compact. On the one hand, since all the leaves of $\mathcal{F}$ are compact, there exists a submersion $f$ : $M \rightarrow \mathbb{R}$ whose level sets are the leaves of $\mathcal{F}$. On the other hand, a compact spacelike hypersurface in a globally hyperbolic spacetime is a Cauchy hypersurface (see e.g. [25]); hence the leaves of $\mathcal{F}$ are Cauchy hypersurfaces. It follows that $f$ is strictly monotonous along every causal curve; hence $f$ or $-f$ is a time function. In particular, the leaves of $\mathcal{F}$ are the level sets of a time function.

A time function $t: M \rightarrow I \subset \mathbb{R}$ is a CMC time function if the level set $t^{-1}(a)$ is a CMC Cauchy hypersurface whose mean curvature is equal to $a$, for every $a \in I$.

REMARK 1.2. To admit a CMC time function is a stronger property than to admit a CMC foliation with compact leaves. The key difference is the following :

- if $t: M \rightarrow I \subset \mathbb{R}$ is a CMC time function then the mean curvature of the level set $t^{-1}(a)$ is equal to $a$. In particular, the mean curvature of the level sets of $t$ increases strictly along future directed timelike curves.

- if $\mathcal{F}$ is a CMC foliation with compact leaves, then the leaves of $\mathcal{F}$ are the level sets of a time function $t: M \rightarrow I \subset \mathbb{R}$, but the mean curvature of the leaf $t^{-1}(a)$ does not necessarily increase strictly with $a$. As a dramatic example, there are CMC foliations all leaves of which have the same mean curvature.

In contrast to the situation for CMC foliations, a globally defined CMC time function with compact level sets is unique, even if the timelike convergence condition 
fails to hold. The proof is a straightforward application of the maximum principle, see $[14, \S 2]$ for details. Similarly, we will prove that CMC time functions in constant sectional curvature MGHC spacetimes are automatically real analytic (see Proposition 4.11) whereas this is not necessarily the case for CMC foliations (see e.g. Proposition 17.5 and Remark 17.7, item 2 and 3).

1.4. Statements of results. A spacetime $(M, g)$ is said to be causally complete if every causal geodesic is complete. It is said to be past (resp. future) complete if every past-directed (resp. future-directed) causal geodesic ray is complete.

1.4.1. The flat case. It is a basic fact that if a flat MGHC spacetime $(M, g)$ is causally complete, then it is a quotient of the Minkowski space $\mathbb{R}^{1, n-1}$ (see e.g. [11]). In this case $M$ is foliated by flat, totally geodesic Cauchy hypersurfaces. Therefore we may focus on the case when $M$ is causally incomplete. Without loss of generality, assume that $M$ is past incomplete. Then $M$ is future complete, and is the quotient of a convex strict subset of $\mathbb{R}^{1, n-1}$ by a group of isometries acting freely and properly discontinuously. This subset is in fact a future regular domain $E^{+}(\Lambda)$, cf. definition 6.2 .

The first result characterizes the generalized mean curvature of the level sets of the cosmological time function in a regular domain.

THEOREM 1.3. Consider a (future complete flat) regular domain $E^{+}(\Lambda)$ in $\mathbb{R}^{1, n-1}$, and the associated cosmological time $\tau: E^{+}(\Lambda) \rightarrow(0,+\infty)$. Then, for every $a \in(0,+\infty)$, the level hypersurface $S_{a}=\tau^{-1}(a)$ has generalized mean curvature bounded from below by $-\frac{1}{a}$, and from above by $-\frac{1}{(n-1) a}$.

Our conventions for second fundamental form and mean curvature are such that the future hyperboloids in Minkowski space have negative mean curvature with respect to the future directed normal (see subsection 1.3). Clearly, Theorem 1.3 holds for quotients of regular domains, and such spaces therefore have a crushing singularity, since the level sets of the cosmological time function provide a sequence of Cauchy hypersurfaces with uniformly diverging mean curvature.

For the case of spacetimes with compact Cauchy hypersurface, a standard barrier argument yields existence of a CMC foliation.

TheOREM 1.4. Let $(M, g)$ be a flat MGHC spacetime.

1. If $(M, g)$ is both past and future complete then it does not admit any globally defined CMC time function, but it admits a unique CMC foliation.

2. If $(M, g)$ is future complete, then it admits a globally defined CMC time function $\tau_{c m c}: M \rightarrow I$ where $I=(-\infty, 0)$. Furthermore, the CMC and cosmological times are comparable:

$$
\tau \leq-\frac{1}{\tau_{c m c}} \leq(n-1) \tau .
$$

3. A similar statement, but with a time range $I=(0,+\infty)$, is true in the past complete case.

In all cases, these foliations are analytic.

REMARK 1.5. This result is not new. It was proved in [9] in the $2+1$ dimensionsal case, assuming the existence of one CMC Cauchy hypersurface. In [1], a proof was given for the case of spacetimes with hyperbolic spatial topology. Finally, it has been 
observed in [11], that the general case follows from the classification of flat MGHC spacetimes.

The proof provided here is conceptually much simpler that the arguments given in the above mentioned papers. More importantly, as we will see, this proof that can be adapted to the general constant curvature case.

Furthermore, the asymptotic behavior of the geometry on the level sets of the cosmological time function is intimately related to the geometry of the singularity itself, i.e. the boundary of the universal cover of the spacetime. For example, we are able to prove that the induced metric on the level sets of the CMC time function and the induced metric on the level sets of the cosmological time function are bilipschitz (with uniform Lipschitz constants). This result was improved very recently by Belraouti $([18])$ who was able to prove the conjecture of Benedetti and Guadagnini [19] that the limit of the geometry of the level sets of the CMC time function in the Gromov sense is the same as the limit of the geometry of the level sets of the cosmological time function. In the $2+1$ dimensional case, this limit can be identified as a point on the Thurston boundary of Teichmuller space. While one expects the limiting geometry of the cosmological time levels to be the same as the CMC time levels in general, there is not yet a clear identification of the limiting geometry except in the $2+1$ dimensional flat case. Observe that Belraouti's result is valid not only for $\mathrm{CMC}$ time function, but more generally for every time function with convex level sets.

REMARK 1.6. There is no compactness condition on Cauchy hypersurfaces in Theorem 1.3. However, a direct proof of existence of CMC hypersurfaces given barriers requires compactness. In a noncompact situation, it is necessary to consider a sequence of Plateau problems, following ideas developed in [46]. It is natural to ask whether any flat regular domain has a CMC foliation. In particular, given two level hypersurfaces of the cosmological time function with mean curvatures bounded above and below by $c$, is there a CMC hypersurface with mean curvature $c$ between them? Similarly, given an isometry group of a regular domain, does there exist CMC hypersurfaces, or CMC foliations, invariant under the isometry group action?

1.4.2. The anti-de Sitter case. The fact that the timelike convergence condition holds strictly in anti-de Sitter spacetimes (i.e. spacetimes with constant negative sectional curvature) simplifies the analysis of CMC time functions. We shall prove the following result:

THeOREM 1.7 (see $\S 13)$. Let $(M, g)$ be a MGHC spacetime with negative constant sectional curvature. Then $(M, g)$ admits a globally defined $C M C$ time function $\tau_{c m c}$ : $M \rightarrow(-\infty, \infty)$.

REMARK 1.8. Theorem 1.7 was already proved in [14] in the particular case where $\operatorname{dim}(M)=3$. The proof provided in [14] uses some sophisticated tools, such as the so-called Moncrief flow on the cotangent bundle of the Teichmüller space, which are very specific to the case where $\operatorname{dim}(M)=3$.

1.4.3. The de Sitter case. In de Sitter spacetimes, i.e. spacetimes of constant positive sectional curvature, the timelike convergence condition fails to hold, and due to this fact the problem of existence of CMC time functions is most difficult in this case. Although they are quite delicate to deal with, MGHC de Sitter spacetimes 
are very abundant and easy to construct. Any compact conformally flat Riemannian manifold gives rise by means of a natural suspension process to a MGHC de Sitter spacetime, and vice-versa. This classification is essentially due to K. Scannell (for more details, see section 14.1). All of theses spaces are (at least) future complete or past complete. According to the nature of the holonomy group of the associated conformally flat Riemannian manifold, i.e. the representation of its fundamental group into the Möbius group, MGHC de Sitter spacetimes split into three types: elliptic, parabolic and hyperbolic (reminiscent of the same classification in Riemannian geometry). Elliptic and parabolic de Sitter spacetimes admit a simple characterization.

- Every elliptic de Sitter spacetime is the quotient of the whole de Sitter space by a finite group of isometries.

- Up to a finite cover, every parabolic dS spacetime is the quotient of some open domain of the de Sitter by a finite rank abelian group of isometries of parabolic type.

Using these geometrical descriptions, it is quite easy to prove that elliptic and parabolic spacetimes do not admit any CMC time function, but admit CMC foliations: More precisely, one has the following results:

Proposition 1.9 (see $\S 17.2)$. Let $(M, g$ ) be an elliptic de Sitter MGHC spacetime. Then, $(M, g)$ admits no CMC time function, but it admits (at least) a CMC foliation. More precisely:

1. if $(M, g)$ is isometric to the whole de Sitter space, it admits infinitely many CMC foliations.

2. if $(M, g)$ is isometric to a quotient of the de Sitter space by a non-trivial group, then there is a unique CMC foliation. Moreover, every CMC Cauchy hypersurface surface in $(M, g)$ is a leaf of this $C M C$ foliation.

Proposition 1.10 (see $\S 17.3$ ). If $(M, g)$ is parabolic, then it admits no $C M C$ time function, but has a unique CMC-foliation. Moreover, every CMC Cauchy surface in $(M, g)$ is a leaf of this CMC foliation.

"Most" de Sitter MGHC spacetimes are hyperbolic. Our last result, even if nonoptimal, tends to show that these spacetimes "usually" admit CMC time functions:

TheOREM 1.11 (see $\S 17.1)$. Let $(M, g)$ be a MGHC hyperbolic de Sitter spacetime. After reversal of time, we can assume that $M$ is future complete. Then, $(M, g)$ admits a partially defined CMC time function $\tau_{c m c}: U \rightarrow I$ where $U$ is a neighbourhood of the past end of $M$ and $I=(-\infty, \beta)$ for some $\beta \leq-1$. Moreover, $U$ is the whole spacetime $M$ and $\beta=-1$ in the following cases,

1. $(M, g)$ has dimension $2+1$,

2. $(M, g)$ is a almost-fuchsian, i.e. contains a Cauchy hypersurface with all principal curvatures $<-1$.

REMARK 1.12. Theorem 1.11 is sharp in the following sense: for any $n \geq 4$, we will give examples of $n$-dimensional de Sitter MGHC spacetimes which do not admit any global CMC time function (see section 17.1.3). A proof of Theorem 1.11 in the particular case where $\operatorname{dim}(M)=3$ was given in [15]. This proof relies on a theorem of F. Labourie on hyperbolic ends of 3-dimensional manifolds, and thus, is very specific to the 3-dimensional case.

REMARK 1.13. There is a well-known natural duality between spacelike immersions of hypersurfaces in de Sitter space and immersions of hypersurfaces in the hyperbolic space (see for example $\left[\begin{array}{ll}15 & \S 5.2 .3\end{array}\right]$ ). This correspondance has the remarkable 
property to invert principal curvatures: if $\lambda$ is a principal curvature of the spacelike hypersurface immersed in de Sitter space, then the inverse $\lambda^{-1}$ is a principal curvature of the corresponding hypersurface immersed in the hyperbolic space. The notion of almost-fuchsian manifolds has been introduced by K. Krasnov and J.-M. Schlenker in $[37, \S 2.2]$ for the riemannian case. More precisely, they defined almost-fuchsian hyperbolic manifolds as hyperbolic quasi-fuchsian manifolds containing a closed hypersurface $S$ with principal curvatures in ] $-1,+1\left[\right.$. For every $r>0$, let $S_{r}$ be the surface made of points at oriented distance $r$ from $S$. Then, for $r$ converging to $-\infty$, the principal curvatures of $S_{r}$ all tend to -1 (see [37, Lemma 2.7]). It follows that hyperbolic almost-fuchsian hyperbolic manifolds can be defined more precisely as hyperbolic quasi-fuchsian manifolds containing a closed hypersurface $S$ with principal curvatures in $]-1,0[$. Here we extended the notion of almost-fuchsian manifolds to the de Sitter case, defining (future complete) almost-fuchsian de Sitter spacetimes as MGHC de Sitter spacetimes containing a Cauchy hypersurface admitting principal curvatures in ] $-\infty,-1$ [. It follows from the discussion above that this terminology is consistent with respect to the Krasnov-Schlenker terminology and the duality between de Sitter space and hyperbolic space. Typical examples are fuchsian spacetimes and small deformations thereof (see Remark 17.2).

1.5. Contents of the paper. The existence of CMC foliations will be obtained by the classical barrier method, using the level sets of the cosmological time function as barriers. Henceforth the proof of our results essentially amounts to analysing the cosmological time function of MGHC spacetimes with constant curvature, in order to prove the desired curvature estimates.

The paper is divided into four parts. In the first part (sections 2 to 5), we review some general facts on the cosmological time functions, the barrier method and $(G, X)$-structure. The three remaining parts correspond to the three possible signs for the curvature of the spacetimes : flat spacetimes (sections 6 to 9), anti-de Sitter spacetimes (sections 10 to 13) and de Sitter spacetimes (section 14 to 17). Each part is itself divided in four sections. In the first section, we give a description of a MGHC spacetime with constant curvature as the quotient of a convex open subset of the Minkowski, the anti-de Sitter or the de Sitter space by a discrete isometry group. In the second section, we give a description of the cosmological time function of the spacetime under consideration. In the third part, we prove some estimates for the mean curvature of the level sets of the cosmological time function. Finally, in the fourth part, we prove the existence of a CMC foliation and/or a CMC time function.

2. Cosmological time functions. In any spacetime $(M, g)$, one can define the cosmological time function, see [5], as follows:

Definition 2.1. The cosmological time function of a spacetime $(M, g)$ is the function $\tau: M \rightarrow[0,+\infty]$ defined by

$$
\tau(x)=\operatorname{Sup}\left\{L(c) \mid c \in \mathcal{R}^{-}(x)\right\}
$$

where $\mathcal{R}^{-}(x)$ is the set of past-oriented causal curves starting at $x$, and $L(c)$ is the lorentzian length of the causal curve $c$.

This function is in general badly behaved. For example, in the case of Minkowski space, the cosmological time function is everywhere infinite.

Definition 2.2. A spacetime $(M, g)$ has regular cosmological time function $\tau$ if 
1. $M$ has finite existence time, i.e. $\tau(x)<\infty$ for every $x$ in $M$,

2. for every past-oriented inextendible causal curve $c:[0,+\infty) \rightarrow M$, $\lim _{t \rightarrow \infty} \tau(c(t))=0$.

In [5], Andersson, Galloway and Howard have proved that spacetimes whose cosmological time function is regular enjoy many nice properties.

THEOREM 2.3. If a spacetime $(M, g)$ has regular cosmological time function $\tau$, then

1. $M$ is globally hyperbolic,

2. $\tau$ is a time function, i.e. $\tau$ is continuous and is strictly increasing along future-oriented causal curves,

3. for each $x$ in $M$, there is a future-oriented timelike geodesic $c:(0, \tau(x)] \rightarrow M$ realizing the distance from the "initial singularity", that is, c has unit speed, is maximal on each segment, and satisfies:

$$
c(\tau(x)))=x \text { and } \tau(c(t))=t \text { for every } t
$$

4. $\tau$ is locally Lipschitz, and admits first and second derivative almost everywhere.

REMARK 2.4. Similarly, for every spacetime $(M, g)$, one may define the reverse cosmological time function of $(M, g)$. This is the function $\widehat{\tau}: M \rightarrow[0,+\infty]$ defined by

$$
\widehat{\tau}(x)=\operatorname{Sup}\left\{L(c) \mid c \in \mathcal{R}^{+}(x)\right\},
$$

where $\mathcal{R}^{+}(x)$ is the set of future-oriented causal curves starting at $x$, and $L(c)$ the lorentzian length of the causal curve $c$. Then one may introduce the notion of spacetime with regular reverse cosmological time function, and prove a result analogous to Theorem 2.3.

3. Generalized mean curvature. If $S$ is a spacelike hypersurface in a spacetime $(M, g)$, then the second fundamental form (also known as the extrinsic curvature) of $S$ at a point $x$ is defined as $\operatorname{II}(X, Y)=\left\langle\nu, \nabla_{X} Y\right\rangle=-\left\langle\nabla_{X} \nu, Y\right\rangle$ where $X, Y$ are tangent vectors to $S$ at $x$ and $\nu$ is the future oriented timelike normal of $S$ (with lorentzian norm -1). The mean curvature is defined in terms of the trace of II with respect to the induced metric as $H_{S}=\operatorname{trII} /(n-1)$. This definition requires $S$ to be at least $C^{2}$. Nevertheless, in certain cases, one can give a meaning to the assertion "a topological hypersurface has mean curvature bounded from below (or above) by some constant $c$ ". A definition of this notion for rough spacelike hypersurfaces was given in [6, Definition 3.3], making use of the notion of supporting hypersurfaces with onesided Hessian bound. The following definition, which does not include the one-sided Hessian bound, is sufficient for our purposes in this paper. We will say that $S$ is a $C^{0}$-spacelike hypersurface in $M$ if for each $x \in S$, there is a neighborhood $U$ of $x$ so that $S \cap U$ is edgeless and acausal in $U$, see [6, Definition 3.1].

Definition 3.1. Let $S$ be a $C^{0}$-spacelike hypersurface in a spacetime $(M, g)$. Given a real number $c$, we will say that $S$ has generalized mean curvature bounded from above by $c$ at $x$, denoted $H_{S}(x) \leq c$, if there is a geodesically convex open neighborhood $V$ of $x$ in $M$ and a smooth spacelike hypersurface $\mathbb{S}_{x}^{-}$in $V$ such that : $-x \in \mathbb{S}_{x}^{-}$and $\mathbb{S}_{x}^{-}$is contained in the past of $S \cap V$ (in $V$ ), 
- the mean curvature of $\mathbb{S}_{x}^{-}$at $x$ is bounded from above by $c$.

Similarly, we will say that $S$ has generalized mean curvature bounded from below by $c$ at $x$, denoted $H_{S}(x) \geq c$, if, there is a geodesically convex open neighborhood $V$ of $x$ in $M$ and a smooth spacelike hypersurface $\mathbb{S}_{x}^{+}$in $V$ such that :

- $x \in \mathbb{S}_{x}^{+}$and $\mathbb{S}_{x}^{+}$is contained in the past of $S \cap V$ (with respect to $V$ ),

- the mean curvature of $\mathbb{S}_{x}^{+}$at $x$ is bounded from below by $c$.

We will write $H_{S} \geq c$ and $H_{S} \leq c$ to denote that $S$ has generalized mean curvature bounded from below respectively above by $c$ for all $x \in S$.

REMARK 3.2. Let $S$ be a smooth spacelike hypersurface in a spacetime $(M, g)$, and $c$ be a real number. If $H_{S} \leq c$ or $H_{S} \geq c$ in the sense of the definition above, then the maximum principle, see Proposition 4.4 below, implies that the same bounds hold in terms of the usual sense.

REMARK 3.3. Let $S$ be a $C^{0}$-spacelike hypersurface, and let $x$ be a point of $S$. Assume that there exists two numbers $c^{-}, c^{+}$such that $S$ has generalized mean curvature bounded from below by $c^{-}$and from above by $c^{+}$at $x$. Then $S$ has a tangent plane at $x$. Indeed, the point $x$ belongs to two smooth hypersurfaces $\mathbb{S}_{x}^{-}$and $\mathbb{S}_{x}^{+}$which are (locally) respectively in the past and in the future $S$. In particular, $\mathbb{S}_{x}^{-}$is locally in the past of $\mathbb{S}_{x}^{+}$. This implies that the tangent hyperplane of $\mathbb{S}_{x}^{-}$at $x$ coincides with the tangent hyperplane of $\mathbb{S}_{x}^{+}$. And since $S$ is between $\mathbb{S}_{x}^{-}$and $\mathbb{S}_{x}^{+}$, this hyperplane is also tangent to $S$.

4. From barriers to CMC time functions. In this section, we consider a $n$-dimensional, $n \geq 3$, maximal globally hyperbolic spacetime $(M, g)$ with compact Cauchy hypersurfaces and constant curvature equal to $k$. We emphasize that many of the proofs that we give are not valid without the assumption that $M$ has compact Cauchy surfaces. Recall that $(M, g)$ has curvature $k$ if the Riemann tensor satisfies

$$
\langle\operatorname{Riem}(X, Y) Y, X\rangle=k\left(\langle X, X\rangle\langle Y, Y\rangle-\langle X, Y\rangle^{2}\right)
$$

for any vector fields $X, Y$. Then the Ricci tensor satisfies Ric $=(n-1) k g$. We will define a notion of sequence of asymptotic barriers, and prove (using quite classical arguments) that $(M, g)$ admits a CMC time function provided that it admits a sequence of asymptotic barriers.

Definition 4.1. Let $c$ be a real number. A pair of $c$-barriers is a pair of $C^{0}$ spacelike Cauchy hypersurfaces $\left(\Sigma^{-}, \Sigma^{+}\right)$in $M$ such that

$-\Sigma^{+}$is in the future of $\Sigma^{-}$,

$-H_{\Sigma^{-}} \leq c \leq H_{\Sigma^{+}}$in the sense of definition 3.1.

Definition 4.2. Let $\alpha$ be a real number. A sequence of asymptotic past $\alpha$ barriers is a sequence of $C^{0}$-spacelike Cauchy hypersurfaces $\left(\Sigma_{m}^{-}\right)_{m \in \mathbb{N}}$ in $M$ such that

$-\Sigma_{m}^{-}$tends to the past end of $M$ when $m \rightarrow+\infty$ (i.e. given any compact subset $K$ of $M$, there exists $m_{0}$ such that $K$ is in the future of $\Sigma_{m}^{-}$for every $\left.m \geq m_{0}\right)$,

$-a_{m}^{-} \leq H_{\Sigma_{m}^{-}} \leq a_{m}^{+}$, where $a_{m}^{-}$and $a_{m}^{+}$are real numbers such that $\alpha<a_{m}^{-} \leq a_{m}^{+}$, and such that $a_{m}^{+} \rightarrow \alpha$ when $m \rightarrow+\infty$.

Similarly, a sequence of asymptotic future $\beta$-barriers is a sequence of $C^{0}$-spacelike Cauchy hypersurfaces $\left(\Sigma_{m}^{+}\right)_{m \in \mathbb{N}}$ in $M$ such that

$-\Sigma_{m}^{+}$tends to the future end of $M$ when $m \rightarrow+\infty$, 
$-b_{m}^{-} \leq H_{\Sigma_{m}^{+}} \leq b_{m}^{+}$, where $b_{m}^{-}$and $b_{m}^{+}$are real numbers such that $b_{m}^{-} \leq b_{m}^{+}<b$, and such that $b_{m}^{-} \rightarrow \beta$ when $m \rightarrow+\infty$.

TheOREM 4.3. Let $(M, g)$ be an $n$-dimensional, $n \geq 3$, maximal globally hyperbolic spacetime, with compact Cauchy hypersurfaces and constant curvature $k$, and such that $(M, g)$ admits a sequence of asymptotic past $\alpha$-barriers and a sequence of asymptotic future $\beta$-barriers. If $k \geq 0$, assume moreover that $(\alpha, \beta) \cap[-\sqrt{k}, \sqrt{k}]=\emptyset$. Then, $(M, g)$ admits a CMC time function $\tau_{c m c}: M \rightarrow(\alpha, \beta)$.

Theorem 4.3 follows easily from known facts in case the barriers are smooth, and introducing $C^{0}$ barriers is not difficult given the results above. Nevertheless, since we are not aware of a reference for this precise statement, we include a proof below. The following are the two main technical steps in the proof. In the case of smooth barriers and hypersurfaces, they were proved in this formulation by Gerhardt [33].

- a proposition which states that any CMC hypersurface of mean curvature $c^{\prime}$ lies in the future of any CMC hypersurface of mean curvature $c$ whenever $c^{\prime}>c$ (Proposition 4.6);

- a theorem which ensures the existence of a Cauchy hypersurface of constant mean curvature $c$, assuming the existence of a pair of $c$-barriers (Theorem 4.9).

Let us start with a slight generalization of the classical maximum principle.

Proposition 4.4. Let $\Sigma$ and $\Sigma^{\prime}$ be two $C^{0}$-spacelike hypersurfaces. Assume that these hypersurfaces have one point $x$ in common, and assume that $\Sigma$ is in the past of $\Sigma^{\prime}$. Assume that $\Sigma$ has generalized mean curvature bounded from above by $c$ at $x$, and $\Sigma^{\prime}$ has generalized mean curvature bounded from below by $c^{\prime}$ at $x$. Then $c \geq c^{\prime}$.

Remark 4.5. Proposition 4.4, which may be viewed as a comparison principle, follows from the strong maximum principle for $C^{0}$ hypersurfaces satisfying a one-sided Hessian bound, see [6, Theorem 3.6]. The notion of generalized curvature we are using here does not include this requirement and we therefore include the simple proof of the proposition.

Proof. Since $\Sigma$ has generalized mean curvature bounded from above by $c$ at $x$, there exists a smooth spacelike hypersurface $S_{x}$ such that $x \in S_{x}, S_{x}$ is in the past of $\Sigma$ and the mean curvature of $S_{x}$ at $x$ is at most $c$. Similarly, there exists a smooth spacelike hypersurface $S_{x}^{\prime}$ such that $x \in S_{x}^{\prime}, S_{x}^{\prime}$ is in the future of $\Sigma^{\prime}$ and the mean curvature of $S_{x}^{\prime}$ at $x$ is at least $c^{\prime}$. Since $\Sigma$ is in the past of $\Sigma^{\prime}$, this implies that $S_{x}$ is in the past of $S_{x}^{\prime}$. And since the point $x$ belongs to both $S_{x}$ and $S_{x}^{\prime}$, we deduce that $S_{x}$ and $S_{x}^{\prime}$ share the same tangent hyperplane at $x$. Now the classical maximum principle can be applied to show that $c \geq c^{\prime}$.

The following result was proved by Gerhardt for the case of spacetimes with a lower bound on the Ricci curvature on timelike vectors, see [33, Lemma 2.1].

Proposition 4.6. Let $(M, g)$ be an n-dimensional, $n \geq 3$, maximal globally hyperbolic spacetime, with compact Cauchy hypersurfaces and constant curvature $k$. Let $\Sigma$ and $\Sigma^{\prime}$ be two smooth Cauchy hypersurfaces in $M$. Assume that $H_{\Sigma} \leq c$ and $H_{\Sigma^{\prime}} \geq c^{\prime}$, with $c \leq c^{\prime}$. If $k$ is non-negative, assume moreover that $c<-\sqrt{k}$ or that $c^{\prime}>\sqrt{k}$. Then $\Sigma^{\prime}$ is in the future of $\Sigma$.

We will give a proof of Proposition 4.6 below, as we shall make use of some of the details in the proof of Theorem 4.3. 
Let $\Sigma_{0}$ be a smooth Cauchy hypersurface with future unit normal $\nu_{0}$. Recall that the orbit of the Gauss flow of smooth Cauchy hypersurface $\Sigma_{0}$ in the direction $\nu_{0}$ consists of the Cauchy hypersurfaces $\Sigma_{t}=F_{t}\left(\Sigma_{0}\right)$ where $F: I \times \Sigma_{0} \rightarrow M$ is defined as $F_{t}(x)=\exp _{x}\left(t \nu_{0}\right)$ for $x \in \Sigma_{0}$, for $t \in I$. Here $I$ is the maximal time interval where $F_{t}$ is regular. The core of the proof of Proposition 4.6 is the following standard comparison lemma, see for example [7, corollary 2.4].

LEMMA 4.7. We consider the orbit $\left(\Sigma_{t}\right)_{t \in I}$ of a smooth Cauchy hypersurface $\Sigma_{0}$ under the Gauss flow. We consider a geodesic $\gamma$ which is orthogonal to the $\Sigma_{t}$ 's, and we denote by $p(t)$ the point of intersection of the geodesic $\gamma$ with the hypersurface $\Sigma_{t}$. The mean curvature $H(t)$ of $\Sigma_{t}$ at $p(t)$ satisfies the differential inequality

$$
\frac{d H(t)}{d t} \geq(n-1)\left(H(t)^{2}-k\right)
$$

Proof of Proposition 4.6. Assume that $\Sigma^{\prime}$ is not in the future of $\Sigma$. Then, we can consider a future-directed timelike geodesic segment $\gamma$ going from a point of $\Sigma^{\prime}$ to a point of $\Sigma$ having maximal length among all such geodesic segments. It is well-known that $\gamma$ is orthogonal to both $\Sigma^{\prime}$ and $\Sigma$, and that there is no focal point to $\Sigma^{\prime}$ or $\Sigma$ along $\gamma$ (see e.g. [36, Proposition 4.5.9]). We will denote by $p^{\prime} \in \Sigma^{\prime}$ and $p \in \Sigma$ the ends of $\gamma$, and by $\delta$ the length of $\gamma$.

If $k$ is non-negative, we have to distinguish two differents cases, according to whether $c^{\prime}>\sqrt{k}$ or $c<-\sqrt{k}$. Let us consider the first case. Since there is no focal point to $\Sigma^{\prime}$ along $\gamma$, the image $\Sigma_{t}^{\prime}$ of $\Sigma^{\prime}$ by the time $t$ of the Gauss flow is well-defined for $t \in[0, \delta]$ in a neighbourhood of $\gamma$. Denote by $p^{\prime}(t)$ the point of intersection of the hypersurface $\Sigma_{t}^{\prime}$ with the geodesic segment $\gamma$, and by $H^{\prime}(t)$ the mean curvature of $\Sigma_{t}^{\prime}$ at $p^{\prime}(t)$. By Lemma 4.7, $t \mapsto H^{\prime}(t)$ satisfies the differential inequality $\frac{d H^{\prime}}{d t} \geq(n-1)\left(H^{\prime 2}-k\right)$. This implies that $H^{\prime}$ increases along $\gamma$ (note that $H^{\prime}(t)^{2}$ is strictly greater than $k$ for every $t$, since $H^{\prime}(0)=c^{\prime}>\sqrt{k}$ by assumption and since $H^{\prime}(t)$ increases). In particular, we have $H^{\prime}(\delta)>H^{\prime}(0)=c^{\prime}$. But now, recall that, by definition of $\Sigma_{\delta}^{\prime}$, every point of $\Sigma_{\delta}^{\prime}$ in a neighbourhood of $\gamma(\delta)=p$ is at distance exactly $\delta$ of $\Sigma^{\prime}$. Also recall that $\gamma$ is the longest geodesic segment joining a point of $\Sigma^{\prime}$ to a point of $\Sigma$. This implies that $\Sigma$ is in the past of $\Sigma_{\delta}^{\prime}$. Hence, by Proposition 4.4, the mean curvature of $\Sigma$ at $p$ is bounded from below by the mean curvature of $\Sigma_{\delta}^{\prime}$, which itself is strictly greater than the mean curvature of $\Sigma^{\prime}$. This contradicts the assumption $c \leq c^{\prime}$.

The proof is the same in the case where $c<-\sqrt{k}$ (except that one considers the backward orbit of $\Sigma$ for the Gauss flow, instead of the forward orbit of $\Sigma^{\prime}$ ).

REMARK 4.8. Proposition 4.6 implies that, for every $c \in \mathbb{R} \backslash[-\sqrt{k}, \sqrt{k}]$, there exists at most one Cauchy hypersurface in $M$ with constant mean curvature equal to c. In particular, for any open interval $(\alpha, \beta)$, which if $k \geq 0$ satisfies the condition $(\alpha, \beta) \cap[-\sqrt{k}, \sqrt{k}]=\emptyset$, there exists at most one function $\tau_{c m c}: M \rightarrow(\alpha, \beta)$ such that $\tau_{c m c}^{-1}(c)$ is a smooth Cauchy hypersurface with constant mean curvature equal to $c$ for every $c \in(\alpha, \beta)$. Note that we are not assuming here that $\tau_{c m c}$ is a time function (recall that, if $\tau_{c m c}$ is a time function, then it is automatically unique, without any assumption on $(\alpha, \beta))$.

Further, it is easy to see using a maximum principle argument, that in the standard de Sitter space with topology $\mathbb{S}^{n-1} \times \mathbb{R}$ and curvature $k>0$, there is no Cauchy hypersurface with mean curvature $c \in \mathbb{R} \backslash[-\sqrt{k}, \sqrt{k}]$. Therefore Proposition 4.6 is vacuous in this case. 
TheOREM 4.9. Let $(M, g)$ be an $n$-dimensional, $n \geq 3$, maximal globally hyperbolic spacetime, with compact Cauchy hypersurfaces. Let $c$ be any real number, and assume that there exists a pair of c-barriers $\left(\Sigma^{-}, \Sigma^{+}\right)$in $M$. Then, there exists a smooth Cauchy hypersurface $\Sigma$ with constant mean curvature equal to $c$. Moreover, $\Sigma$ is in the future of $\Sigma^{-}$and in the past of $\Sigma^{+}$.

Proof. The result is proved e.g. in [32] in the case where the barriers $\Sigma^{-}$and $\Sigma^{+}$ are smooth. The only way the barriers $\Sigma^{-}$and $\Sigma^{+}$are used in Gerhardt's proof is via the maximum principle (to show that a family of Cauchy hypersurfaces whose mean curvature approaches $c$ cannot "escape to infinity"). Since the maximum principle is still valid for $C^{0}$ hypersurfaces (Proposition 4.4), Gerhardt's proof also applies in the case where the barriers are not smooth.

Proof of Theorem 4.3. We consider a sequence $\left(\Sigma_{m}^{-}\right)_{m \in \mathbb{N}}$ of asymptotic past $\alpha$ barriers, and a sequence $\left(\Sigma_{m}^{+}\right)_{m \in \mathbb{N}}$ of asymptotic future $\beta$-barriers.

Construction of the function $\tau_{c m c}$. Fix $c \in(\alpha, \beta)$. For $m$ large enough, the pair of Cauchy hypersurfaces $\left(\Sigma_{m}^{-}, \Sigma_{m}^{+}\right)$is a pair of $c$-barriers. Thus, by Theorem 4.9, for any $c \in(\alpha, \beta)$, there exists a Cauchy hypersurface $S_{c}$ with constant mean curvature equal to $c$. Proposition 4.6 implies that the $S_{c}$ 's are pairwise disjoint, and that $S_{c}$ is in the past of $S_{c^{\prime}}$ if $c<c^{\prime}$ (let us call this "property $(\star)$ ").

Now, let us prove that the set $\bigcup_{c \in(\alpha, \beta)} S_{c}$ is connected. Assume the contrary. Because of property $(\star)$, there are only two possible cases :

(i) there exists $c_{0} \in(\alpha, \beta)$ such that $\bigcup_{c>c_{0}} I^{+}\left(S_{c}\right) \subsetneq I^{+}\left(S_{c_{0}}\right)$,

(ii) or there exists $c_{0} \in(\alpha, \beta)$ such that $\bigcup_{c<c_{0}} I^{-}\left(S_{c}\right) \subsetneq I^{-}\left(S_{c_{0}}\right)$.

Let us consider, for example, case (i). Using the Gauss flow, we can push the hypersurface $S_{c_{0}}$ towards the future, in order to obtain a Cauchy hypersurface $S_{c_{0}}^{\prime}$ which is in the future of $S_{c_{0}}$, but as close to $S_{c_{0}}$ as we want. In particular, we can assume that $S_{c_{0}}^{\prime}$ is not in the future of $S_{c}$ for any $c>c_{0}$. Moreover, according to Lemma 4.7, the mean curvature of $S_{c_{0}}^{\prime}$ is bounded from below by some number $c_{0}^{\prime}>c_{0}$. But this contradicts Proposition 4.6. Case (ii) can be treated similarly. As a consequence, the set $\bigcup_{c \in(\alpha, \beta)} S_{c}$ is connected. Note that this implies that the hypersurface $S_{c}$ depends continuously on $c$

Now, let us prove that the union $\bigcup_{c \in(\alpha, \beta)} S_{c}$ is equal to the whole $M$. Assume that there exists a point $x \in M \backslash \bigcup_{c \in(\alpha, \beta)} S_{c}$. Since the hypersurface $S_{c}$ depends continuously on $c$, there are only two possible cases :

(i) either $x$ is in the future of $S_{c}$ for every $c \in(\alpha, \beta)$,

(ii) or $x$ is in the past of $S_{c}$ for every $c \in(\alpha, \beta)$.

Now, recall that we have a sequence $\left(S_{m}^{+}\right)_{m \in \mathbb{N}}$ of asymptotic future $\beta$-barriers. By definition, this means that $S_{m}^{+}$has generalized mean curvature bounded from below by some $b_{m}^{-}$and smaller than some $b_{m}^{+}$where $b_{m}^{-} \leq b_{m}^{+}<\beta$ and $b_{m}^{-} \rightarrow_{m \rightarrow \infty} \beta$. Fix some integer $p$. One can find $q>p$ such that $b_{q}^{-}>b_{p}^{+}$. Then $\left(S_{p}^{+}, S_{q}^{-}\right)$is a pair of $b_{p}^{+}$-barriers. By Theorem 4.9, one can find a Cauchy hypersurface with constant mean curvature equal to $b_{p}^{+}$between $S_{p}^{+}$and $S_{q}^{-}$, and by uniqueness (see remark 4.8), this hypersurface is the hypersurface $S_{c}$ for $c=b_{p}^{+}$. In particular, for $c \geq b_{p}^{+}$, the hypersurface $S_{c}$ is in the future of the barrier $S_{p}^{+}$. Now, recall that, by definition of a sequence of asymptotic future barriers, $S_{p}^{+}$tends to the future end of $M$ when $p \rightarrow \infty$. This shows that case (i) cannot happen. Of course, one can exclude case (ii) using similar arguments. Therefore we have proved that $\bigcup_{c \in(\alpha, \beta)} S_{c}=M$.

Now, we can define the function $\tau_{c m c}: M \rightarrow(\alpha, \beta)$ as follows : for every $x \in M$, we set $\tau_{c m c}(x)=c$ where $c$ is the unique number such that $x \in S_{c}$. 
Properties of the function $\tau_{c m c}$. The fact the hypersurface $S_{c}$ depends continuously on $c$ implies that the function $\tau_{c m c}$ is continuous. The fact that the hypersurface $S_{c^{\prime}}$ is in the strict future of the hypersurface $S_{c}$ when $c^{\prime}>c$ implies that the function $\tau_{c m c}$ is strictly increasing along any future directed timelike curve. Hence, $\tau_{c m c}$ is a time function.

REMARK 4.10. Using the same arguments as above, one can prove the following result:

Let $(M, g)$ be an $n$-dimensional, $n \geq 3$, maximal globally hyperbolic spacetime, with compact Cauchy hypersurfaces and constant curvature $k$. Assume that $(M, g)$ admits a sequence of asymptotic past $\alpha$-barriers. If $k \geq 0$, assume moreover that $\alpha \notin[-\sqrt{k}, \sqrt{k}]$. Then, $(M, g)$ admits a partially defined CMC time function $\tau_{c m c}: U \rightarrow(\alpha, \beta)$ where $U$ is a neighbourhood of the past end of $M$ (i.e. the past of a Cauchy hypersurface in $M)$ and $\beta$ is a real number greater than $\alpha$.

Proposition 4.11. Let $(M, g)$ be an $n$-dimensional, $n \geq 3$, maximal globally hyperbolic spacetime, with compact Cauchy hypersurfaces and constant curvature $k$. Suppose that there exists a function $\tau_{c m c}: M \rightarrow(\alpha, \beta)$ such that $\tau_{c m c}^{-1}(c)$ is a Cauchy hypersurface with constant mean curvature equal to $c$ for every $c \in(\alpha, \beta)$. Assume moreover that one of the following hypotheses is satisfied:

- $\tau_{c m c}$ is a time function,

- the curvature $k$ is negative,

- the curvature $k$ is non-negative and $(\alpha, \beta) \cap[-\sqrt{k}, \sqrt{k}]=\emptyset$. Then $\tau_{c m c}$ is real analytic.

Sketch of proof. Under the stated conditions, there is exactly one CMC Cauchy hypersurface for each $c \in(\alpha, \beta)$. CMC hypersurfaces in a real analytic spacetime are real analytic, since they are solutions of a quasi-linear elliptic PDE. Given a CMC Cauchy hypersurface $S_{0}$ with mean curvature $c_{0} \in(\alpha, \beta)$, let $u$ be the Lorentz distance to $S_{0}$. For $c$ close to $c_{0}$, a Cauchy hypersurface $S_{c}$ with mean curvature $c$ is a graph over $S_{0}$, defined by the level function $w=\left.u\right|_{S_{c}}$. The function $w$ solves the mean curvature equation $H[w]=c$, which is a quasilinear elliptic system with real analytic dependence on the coefficients. It follows that $S_{c}$ depends in a real-analytic manner on $c$, and that the function $\tau_{c m c}$ is a real analytic function on $M$.

REMARK 4.12. The function $\tau_{c m c}$ is also a time function in the following stronger sense : for every future directed timelike curve $\gamma: I \rightarrow \mathbb{R}$, one has

$$
\frac{d}{d t} \tau_{c m c}(\gamma(t))>0
$$

Indeed, fix such a curve $\gamma$ and some $t_{0} \in I$, let $x_{0}=\gamma\left(t_{0}\right)$ and $c_{0}=\tau_{c m c}\left(x_{0}\right)$. For $t$ small enough, denote by $S_{c_{0}}^{t}$ the image of the hypersurface $S_{c_{0}}$ by the time $t$ of the Gauss flow. Since the derivatives of $\gamma$ are future-oriented timelike vectors, there exists a constant $\lambda_{1}>0$ such that, for $h>0$ small enough, the point $\gamma\left(t_{0}+h\right)$ is in the future of the image of the hypersurface $S_{c_{0}}^{\lambda_{1} . h}$. Now Lemma 4.7 implies that there exists a constant $\lambda_{2}>0$ such that the mean curvature of the hypersurface $S_{c_{0}}^{\lambda_{1} \cdot h}$ is bounded from below by $c_{0}+\lambda_{1} \cdot \lambda_{2} \cdot h$ (for $h$ small enough). Then Proposition 4.6 implies that $S_{c_{0}+\lambda_{1} \cdot \lambda_{2} . h}$ is in the past of $S_{c_{0}}^{\lambda_{1}} \cdot h$. In particular, for $h$ small enough, the point $\gamma\left(t_{0}+h\right)$ is the future of the hypersurface $S_{c_{0}+\lambda_{1} \cdot \lambda_{2} . h}$. In other words, we have $\tau_{c m c}\left(t_{0}+h\right)>c_{0}+\lambda_{1} \cdot \lambda_{2} \cdot h$. This implies $\frac{d}{d t} \tau_{c m c}(\gamma(t))>\lambda_{1} \cdot \lambda_{2}>0$. 
5. Spaces of constant curvature as $(G, X)$-structures. Let $X$ be a manifold and $G$ be a group acting on $X$ with the following property: if an element $\gamma$ of $G$ acts trivially on an open subset of $X$, then $\gamma$ is the identity element of $G$. A $(G, X)$ structure on a manifold $M$ is an atlas $\left(U_{i}, \phi_{i}\right)_{i \in I}$ where

- $\left(U_{i}\right)_{i \in I}$ is a covering of $M$ by open subsets,

- for every $i$, the map $\phi_{i}$ is a homeomorphism from $U_{i}$ to an open set in $X$,

- for every $i, j$, the transition map $\phi_{i} \circ \phi_{j}^{-1}: \phi_{j}\left(U_{i} \cap U_{j}\right) \rightarrow \phi_{i}\left(U_{i} \cap U_{j}\right)$ is the restriction of an element of $G$.

Given a manifold $M$ equipped with a $(G, X)$-structure $\left(U_{i}, \phi_{i}\right)_{i \in I}$, one can construct two important objects: a map $d: \widetilde{M} \rightarrow X$, called developing map, and representation $\rho: \pi_{1}(M) \rightarrow G$, called holonomy representation. The map $d$ is a local homeomorphism (obtained by pasting together some lifts of the $\phi_{i}$ 's) and satisfies the following equivariance property: for every $\widetilde{x} \in \widetilde{M}$ and every $\gamma \in \pi_{1}(M)$, one has $d(\gamma \cdot \widetilde{x})=\rho(\gamma) \cdot d(\widetilde{x})$. The map $d$ is unique up to post-composition by an element of $G$ and the choice of $d$ obviously fully determines the representation $\rho$. In general, $d$ is neither one-to-one, nor onto. A good reference for all these notions is [35].

Now let $(M, g)$ be a $n$-dimensional spacetime with constant curvature $k=0$ (respectively $k=1$ and $k=-1$ ). Then it is well-known that every point in $M$ admits a neighbourhood which is isometric to an open subset of the Minkowski space $\mathbb{R}^{1, n-1}$ (respectively the de Sitter space $\mathrm{dS}_{n}$ and the anti-de Sitter space $\operatorname{AdS}_{n}$ ). In other words, the lorentzian metric on $M$ can be seen as a $(G, X)$-structure, where $X=\mathbb{R}^{1, n-1}$ (respectively $\mathrm{dS}_{n}$ and $\operatorname{AdS}_{n}$ ) and $G=\operatorname{Isom}(X)$. Hence the general theory provides us with a locally isometric developing map $d: \widetilde{M} \rightarrow X$ and a representation $\rho: \pi_{1}(M) \rightarrow \operatorname{Isom}(X)$ such that $d(\gamma \cdot \widetilde{x})=\rho(\gamma) \cdot d(\widetilde{x})$ for every $\widetilde{x} \in \widetilde{M}$ and every $\gamma \in \pi_{1}(M)$. The map $d$ and the representation $\rho$ will play a fundamental role in the proofs of Theorems 1.7 and 1.11.

\section{Description of flat MGHC spacetimes.}

6.1. Flat regular domains. Regular domains in Minkowski space $\mathbb{R}^{1, n-1}$ were first defined by F. Bonsante in [23, 24] (generalizing a construction of G. Mess in the 2+1-dimensional case, see [40]). Here we will use an equivalent definition introduced in [11], since it appears to be slightly more adapted to our purpose. For more details, we refer to section 4.1 of [11].

The importance of flat regular domains for our purpose comes from the fact that they have regular cosmological time function, see Proposition 6.5, and that each flat maximal globally hyperbolic spacetime with compact, or more generally complete, Cauchy hypersurface is a quotient of a flat regular domain, see Theorem 6.7. Thus, the analysis of the singularity of these spacetimes can be carried out by studying the past boundary of flat regular domains. This will be carried out in section 7 .

Definition 6.1. The Penrose boundary $\mathcal{J}_{n-1}$ of the Minkowski spacetime $\mathbb{R}^{1, n-1}$ is the space of null affine hyperplanes of $\mathbb{R}^{1, n-1}$

Let $N$ be an auxiliary euclidean metric on $\mathbb{R}^{1, n-1}$. Let $\mathcal{S}^{n-2}$ be the set of future oriented null elements of $\mathbb{R}^{1, n-1}$ with $N$-norm 1 . Then the map which associates to a pair $(u, a)$ the null hyperplane $H(u, a)=\{x \mid\langle x, u\rangle=a\}$ is a bijection between $\mathcal{S}^{n-2} \times \mathbb{R}$ and $\mathcal{J}_{n-1}$. It defines a topology on $\mathcal{J}_{n-1}$, which coincides with the topology of $\mathcal{J}_{n-1}$ as a homogeneous space under the action of the Poincaré group; $\mathcal{J}_{n-1}$ is then homeomorphic to $\mathbb{S}^{n-2} \times \mathbb{R}$. 
For every element $p$ of $\mathcal{J}_{n-1}$, we denote by $E^{+}(p)$ the future of $p$ in $\mathbb{R}^{1, n-1}$, and by $E^{-}(p)$ the past of of $p$. If $p$ is the null hyperplane $H(u, a)$, then $E^{+}(p)=\{x \mid\langle x, u\rangle<$ $a\}$ and $E^{-}(p)=\{x \mid\langle x, u\rangle>a\}$. They are half-spaces, respectively future-complete and past-complete. For every closed subset $\Lambda$ of $\mathcal{J}_{n-1}$, we define

$$
E^{ \pm}(\Lambda)=\bigcap_{p \in \Lambda} E^{ \pm}(p)
$$

Definition 6.2. A closed subset $\Lambda$ of $\mathcal{J}_{n-1}$ is said to be future regular (resp. past regular) if it contains at least two elements and if $E^{+}(\Lambda)$ (resp. $E^{-}(\Lambda)$ ) is non-empty.

A future complete flat regular domain is a domain of the form $E^{+}(\Lambda)$ where $\Lambda$ is a future regular closed subset of $\mathcal{J}_{n-1}$. Similarly, a past complete flat regular domain is a domain of the form $E^{-}(\Lambda)$ where $\Lambda$ is a past regular closed subset of $\mathcal{J}_{n-1}$. A flat regular domain is a future complete regular domain or a past complete regular domain.

See $\S 4.2$ of [11] where it is proved in particular that this definition of flat regular domains coincides with Bonsante's definition.

REMARK 6.3. A past regular closed set $\Lambda$ is not necessarily future regular. Actually, a closed subset of $\mathcal{J}_{n-1}$ is past regular and future regular if and only if it is compact (and contains at least two points). See Corollary 4.11 in [11].

REMARK 6.4. In the rest of the paper, we will mainly be dealing with a past incomplete, future complete spacetimes, and many statements have an obvious time reversed analog. In the following we will not make any explicit statements concerning the time reversed situation, and leave it to the reader to rephrase the relevant definitions and results.

One of the cornerstones of Bonsante's work on flat regular domains is the following proposition:

Proposition 6.5. Future complete flat regular domains have regular cosmological time.

Proof. See [24, Proposition 4.3 and Corollary 4.4].

\subsection{Flat MGHC spacetimes.}

Proposition 6.6. Let $E^{+}(\Lambda) \subset \mathbb{R}^{1, n-1}$ be a future complete flat regular domain. Let $\Gamma$ be a discrete torsion free group of isometries of Minkoswki spacetime $\mathbb{R}^{1, n-1}$ preserving $E^{+}(\Lambda)$. Then, the action of $\Gamma$ on $E^{+}(\Lambda)$ is free and properly discontinuous, and the quotient space $M_{\Lambda}^{+}(\Gamma)=\Gamma \backslash E^{+}(\Lambda)$ is a globally hyperbolic spacetime with regular cosmological time.

Sketch of proof. The proof that the action is free and properly discontinuous can be found in [11, Proposition 4.16]. The cosmological time $\tau$ is obviously $\Gamma$-invariant. Hence, it induces a map $\hat{\tau}$ on the quotient $M_{\Lambda}^{+}(\Gamma)$. Since inextendible causal curves in $M_{\Lambda}^{+}(\Gamma)$ are projections of causal curves in $E^{+}(\bar{\Lambda})$, the cosmological time on the quotient $M_{\Lambda}^{+}(\Gamma)$ is the map $\hat{\tau}$. It follows easily that $M_{\Lambda}^{+}(\Gamma)$ has regular cosmological time.

\section{Conversely:}

THEOREM 6.7. Every flat MGHC spacetime is the quotient of a flat regular domain or of the entire Minkowski space by a torsion-free discrete subgroup of isometries. More precisely, let $(M, g)$ be a n-dimensional flat $M G H C$ spacetime. 
1. If $(M, g)$ is not past (resp. future) complete, then $(M, g)$ is the quotient of a future (resp. past) complete regular domain in $\mathbb{R}^{1, n-1}$ by a torsion-free discrete subgroup of Isom $\left(\mathbb{R}^{1, n-1}\right)$.

2. If $(M, g)$ is causally complete then it is the quotient of $\mathbb{R}^{n-1,1}$ by a subgroup of $\operatorname{Isom}\left(\mathbb{R}^{1, n-1}\right)$ containing a finite index free abelian subgroup generated by $n-1$ spacelike translations.

Proof. It follows from the classification of flat MGHC spacetimes given in [11]. The result in [11] is more precise: it characterizes up to finite index the possible torsion-free discrete subgroups.

REMARK 6.8. The natural setting for a result like Theorem 6.7 is not really spacetimes with compact Cauchy hypersurfaces, but rather flat maximal globally hyperbolic spacetimes with complete Cauchy hypersurfaces (abbreviation: complete MGH flat spacetimes). Indeed, every flat regular domain admits a complete Cauchy hypersurface ( see [11, Proposition 4.14]). Conversely, according to [11, Theorem 1.1], every complete MGH flat spacetime can be tamely embedded in the quotient of a flat regular domain by a discrete group of isometries of Minkowski except if it is causally complete or if it is an unipotent spacetime. Complete MGH flat spacetimes which are also causally complete are quotients of the entire Minkoswki space $\mathbb{R}^{1, n-1}$ by a commutative discrete group of spacelike translations. Flat unipotent spacetimes are defined and described in $\S 3.3$ of [11] (see also [31]); every flat unipotent spacetime is the quotient of a domain $\Omega \subset \mathbb{R}^{1, n-1}$ by a unipotent discrete subgroup of $\operatorname{Isom}\left(\mathbb{R}^{1, n-1}\right)$, where $\Omega$ is of one of the three following forms: $\Omega=E^{+}(p), \Omega=E^{-}(p)$ or $\Omega=E^{+}(p) \cap E^{-}\left(p^{\prime}\right)$ where $p$ and $p^{\prime}$ are two parallel null hyperplanes.

7. Cosmological time and horizon of flat regular domains. In this section, we consider a future complete flat regular domain $E^{+}(\Lambda)$. We will describe the past horizon, the initial singularity, and the so-called "retraction to the initial singularity" of $E^{+}(\Lambda)$.

7.1. Horizons. According to Proposition 6.5 and Theorem 2.3, $E^{+}(\Lambda)$ is globally hyperbolic. Since $E^{+}(\Lambda)$ is a future complete convex open domain in Minkowski space, its boundary $\mathcal{H}^{-}(\Lambda)$ is a past horizon (and thus enjoys all the known properties of horizons).

Since $\mathcal{H}^{-}(\Lambda)$ is the boundary of a convex domain, it admits support hyperplanes at each of its points. And since $E^{+}(\Lambda)$ is future complete, the future in $\mathbb{R}^{1, n-1}$ of any point $p$ in $\mathcal{H}^{-}(\Lambda)$ is contained in $E^{+}(\Lambda)$. But, timelike hyperplanes containing $p$ all intersect the future of $p$, it then follows that support hyperplanes to $\mathcal{H}^{-}(\Lambda)$ are non-timelike.

Lemma 7.1. Let $p$ be a point of the past horizon $\mathcal{H}^{-}(\Lambda)$ of a future complete flat regular domain $E^{+}(\Lambda)$. Let $C(p) \subset T_{p} X$ be the set of future oriented tangent vectors orthogonal to support hyperplanes to $\mathcal{H}^{-}(\Lambda)$ at $p$. Then $C(p)$ is the convex hull of its null elements. Moreover, the null elements of $C(p)$ are precisely the normals to elements of $\Lambda$ tangent to $\mathcal{H}^{-}(\Lambda)$ at $p$.

Proof. See [24, corollary 4.12] (see also [40, Proposition 11]).

7.2. Retraction to the initial singularity. According to point (3) in Theorem 2.3, for every point $x$ in a flat regular domain there is a unique maximal timelike geodesic ray with future endpoint $x$ realizing the "distance to the initial singularity": we call such a geodesic ray a realizing geodesic for $x$. 
Proposition 7.2. Let $x$ be an element of a future complete flat regular domain $E^{+}(\Lambda)$. Then, there is a unique realizing geodesic for $x$.

Proof. See [24, Proposition 4.3].

Definition 7.3. A unit speed future oriented timelike geodesic $\gamma:[0, T] \rightarrow$ $E^{+}(\Lambda)$ is tight if for every $t$ in $[0, T]$ the restriction $\gamma:[0, t] \rightarrow E^{+}(\Lambda)$ is a realizing geodesic for $\gamma(t)$.

Proposition 7.4. Let $\gamma:[0, T] \rightarrow E^{+}(\Lambda)$ be a unit speed future oriented timelike geodesic with initial point in the past horizon. Then the following assertions are equivalent:

1. $\gamma$ is tight,

2. the derivative of $\gamma$ at 0 is orthogonal to a support hyperplane at $\gamma(0)$ of the past horizon.

Proof. See [24, Proposition 4.3].

Definition 7.5. The initial singularity of a future complete flat regular domain $E^{+}(\Lambda)$ is the set of points in the past horizon admitting at least two support hyperplanes; it will be denoted by $\Sigma^{-}(\Lambda)$.

Proposition 7.6. The map which associates to any point $x$ of a regular domain $E^{+}(\Lambda)$, the initial singularity of the unique realizing geodesic for $x$ is a continuous map taking value in $\Sigma^{-}(\Lambda)$. This map is denoted $r$, and called "retraction to the initial singularity".

Proof. See [24, Proposition 4.3 and 4.12].

\subsection{Description of the retraction map.}

Proposition 7.7. For every $p$ in the past singularity $\Sigma^{-}$, the preimage $r^{-1}(p)$ in $E^{+}(\Lambda)$ is the union of complete timelike geodesic rays with initial point at $p$.

Proof. The Proposition is an immediate corollary of Proposition 7.2 and 7.4.

Corollary 7.8. Let $p$ be an element of the past horizon of $E^{+}(\Lambda)$ such that the convex hull $C(p)$ of the null generators has non-empty interior in the space of timelike tangent vectors at $p$. Then, $r^{-1}(p)$ is open in $E^{+}(\Lambda)$.

8. Flat regular domains: curvature estimates for cosmological time level sets. Let us recall the statement of Theorem 1.3:

THEOREM 1.3. Consider a future complete flat regular domain $E^{+}(\Lambda)$ and the associated cosmological time $\tau: E^{+}(\Lambda) \rightarrow(0,+\infty)$. Then, for every $a \in(0,+\infty)$, the hypersurface $S_{a}=\tau^{-1}(a)$ has generalized mean curvature satisfying $-\frac{1}{a} \leq H_{S_{a}} \leq$ $-\frac{1}{(n-1) a}$.

REMARK 8.1. What is important for us in the proof of Theorem 1.4 is the fact that the hypersurface $S_{a}$ has generalized mean curvature satisfying $\alpha(a) \leq H_{S_{a}} \leq \beta(a)$, where $\alpha(a), \beta(a) \rightarrow-\infty$ when $a \rightarrow 0$, and $\alpha(a), \beta(a) \rightarrow 0$ when $a \rightarrow+\infty$.

Proof. Let $x$ be a point on the level set $S_{a}$. We denote by $\gamma:[0, a] \rightarrow E^{+}(\Lambda)$ the unique realizing geodesic for $x$, with initial point $p=r(x)$. Let $v$ be the future oriented unit speed tangent vector of $\gamma$ at $p$. We denote as before by $C(p)$ the set of vectors in $T_{p} X$ orthogonal to support hyperplanes of the past horizon at $p$. 
Construction of $\mathbb{S}_{x}^{+}$. Define $\mathbb{S}_{x}^{+}$as the hyperboloid $\{z \mid d(p, z)=a\}$. Since $E^{+}(\Lambda)$ is geodesically convex, for any $z$ in $\mathbb{S}_{x}^{+}$the timelike geodesic $(p, z)$ is contained in $E^{+}(\Lambda)$. Hence, its length $a$ is less than $\tau(z)$. The unique realizing geodesic for $z$ must therefore intersect $S_{a}$. Hence, $\mathbb{S}_{x}^{+}$is contained in the future of $S_{a}$. The tangent hyperplane to $\mathbb{S}_{x}^{+}$at $x$ is the hyperplane orthogonal to $c$ at $x$. Hence, $\mathbb{S}_{x}^{+}$is tangent to $S_{a}$ at $x$. Finally, the mean curvature of $\mathbb{S}_{x}^{+}$is obviously $-\frac{1}{a}$ everywhere. As a consequence, $S_{a}$ has generalized mean curvature satisfying $H_{S_{a}} \geq-\frac{1}{a}$.

Construction of $\mathbb{S}_{x}^{-}$. According to Lemma 7.1, the tangent vector $v$ of the realizing geodesic $\gamma$ introduced above, belongs to the convex hull $C(p)$. Let $B$ be a finite subset of the null elements of $C(p)$ such that $v$ lies in the convex hull of $B$. We choose moreover $B$ minimal, i.e. such that for any proper subset $B^{\prime} \subset B, v$ does not belong to the convex hull of $B^{\prime}$. An equivalent statement is that $v$ belongs to the relative interior $\operatorname{Conv}(B)$.

The null hyperplanes $p+w^{\perp}$ for $w$ in $B$ form a finite subset $\Lambda_{B}$ of $\Lambda$. Observe that since the convex hull of $B$ contains the timelike vector $v, B$ contains at least two elements. Hence, $E^{+}\left(\Lambda_{B}\right)$ is a future complete flat regular domain.

Obviously, $E^{+}\left(\Lambda_{B}\right)$ contains $E^{+}(\Lambda)$. Hence $\mathcal{H}^{-}\left(\Lambda_{B}\right)$ is contained in the causal past of $E^{+}(\Lambda)$. Moreover, $E^{+}\left(\Lambda_{B}\right)$ contains the timelike geodesic $\gamma$, and also $x$, and its past horizon $\mathcal{H}^{-}\left(\Lambda_{B}\right)$ contains $p$. According to Lemma 7.1, support hyperplanes to $\mathcal{H}^{-}\left(\Lambda_{B}\right)$ at $p$ are hyperplanes orthogonal to vectors in the convex hull of $B$. In particular, the hyperplane orthogonal to the timelike vector $v$ is a spacelike support hyperplane. It follows that $\gamma$ is a realizing geodesic for $x$ in $E^{+}\left(\Lambda_{B}\right)$. Hence, $\tau_{B}(x)=$ $a$, where $\tau_{B}$ is the cosmological time for $E^{+}\left(\Lambda_{B}\right)$.

Let $S_{B}^{\prime}$ be the level set $\left\{\tau_{B}=a\right\}$ in $E^{+}\left(\Lambda_{B}\right)$, and define $\mathbb{S}_{x}^{-}$as a small open neighborhood of $x$ in $S_{B}^{\prime} \cap E^{+}(\Lambda)$. Let $V$ be a geodesically convex neighborhood of $x$ containing $\mathbb{S}_{x}^{-}$(for example, the Cauchy development of $\mathbb{S}_{x}^{-}$in $E^{+}(\Lambda)$ ). For any $z$ in $S_{B}^{\prime}$ let $c$ be the unique realizing geodesic for $z$ in $E^{+}(\Lambda)$. Since $\mathcal{H}^{-}\left(\Lambda_{B}\right)$ is in the causal past of $\mathcal{H}^{-}(\Lambda)$ there is a past extension of $c$ with past endpoint in $\mathcal{H}^{-}\left(\Lambda_{B}\right)$. Hence, $\tau(z) \leq a$. It follows that $\mathbb{S}_{x}^{-}$lies in the causal past of $S_{a}$ in $V$.

To complete the proof, we must prove that $\mathbb{S}_{x}^{-}$near $x$ is smooth, admits at $x$ the same tangent hyperplane $(x-p)+v^{\perp}$, and that it has constant mean curvature $-\frac{d}{(n-1) a}$ for some integer $1 \leq d \leq n-1$.

Consider $\mathbb{R}^{1, n-1}$ as a vector space, with origin $p=0$. Let $F$ be the vector space spanned by $\operatorname{Conv}(B)$. Then $F$ is a timelike subspace, with dimension $2 \leq k \leq n$, and we have a splitting $\mathbb{R}^{1, n-1}=F \oplus F^{\perp}$. The subspace $F^{\perp}$ is spacelike. Every element of $\Lambda_{B}$ is a null hyperplane containing $F^{\perp}$. It follows easily that $E^{+}\left(\Lambda_{B}\right)$ is the sum $E^{\prime}\left(\Lambda_{B}\right) \oplus F^{\perp}$, where $E^{\prime}\left(\Lambda_{B}\right)=F \cap E^{+}\left(\Lambda_{B}\right)$. For every element $H$ of $\Lambda_{B}, H \cap F$ is a null hyperplane in $F \approx \mathbb{R}^{1, k-1}$.

Let $\Lambda_{B}^{\prime}=\left\{H \cap F \mid H \in \Lambda_{B}\right\}$. Then $\Lambda_{B}^{\prime}$ is a finite subset of the Penrose boundary of $F$. Clearly $E^{\prime}\left(\Lambda_{B}\right)$ is precisely the flat regular domain $E\left(\Lambda_{B}^{\prime}\right) \subset F$. Now we observe that restricting to $F, v$ is in the interior of $\operatorname{Conv}\left(\Lambda_{B}^{\prime}\right)$. Hence, for some small neighborhood $V^{\prime}$ of $x$ in $E\left(\Lambda_{B}^{\prime}\right)$, which can be selected geodesically convex, the image by the retraction $r$ of each point $y$ in $V^{\prime}$ is $p$. Shrinking $V$ if necessary, we can assume that $V$ is contained in $V^{\prime} \oplus F^{\perp}$. According to Corollary $7.8, \mathbb{S}_{x}^{-}$has the form $\mathbb{H} \oplus F^{\perp}$, where $\mathbb{H}$ is the hyperboloid consisting of points in $F$ in the future of $p$ and at lorentzian distance $a$ from $p$. Hence, $\mathbb{S}_{x}^{-}$is smooth and admits at $x$ the same tangent hyperplane than $S_{a}$ (the orthogonal $x+v^{\perp}$ ). Moreover, since $x+F^{\perp}$ is totally geodesic, and since the principal directions of $\mathbb{H}$ are all equal to $-\frac{1}{a}$, the mean curvature of $\mathbb{S}_{x}^{-}$is equal to $-\frac{1}{a} \cdot \frac{d}{n-1}$ where $d=k-1$. This shows that $S_{a}$ has generalized mean curvature 
satisfying $H_{S_{a}} \leq-\frac{1}{(n-1) a}$. $\square$

REMARK 8.2. The proof of Theorem 1.3 shows that the second fondamental form of $\mathbb{S}_{x}^{-}, \mathbb{S}_{x}^{+}$have eigenvalues $-1 / a, 0$ (in the case of $\mathbb{S}_{x}^{-}$) and $-1 / a$ (in the case of $\left.\mathbb{S}_{x}^{+}\right)$. Therefore the level sets of $\tau$ have mean curvature satisfying $-1 / a \leq H_{S_{a}} \leq$ $-1 /((n-1) a)$ with one-sided Hessian bound, as in [26, Definition 3.3], and hence the strong maximum principle for spacelike hypersurfaces given in [6, Theorem 3.6] applies in our situation. However we shall not need the full strength of this result here. See Proposition 4.4 below for the version of the maximum principle which we shall make use of.

The eigenvalue bounds stated in Remark 8.2 allow us to give a more precise characterization of the regularity of the cosmological time function. The Hessian bounds for the height function implied by the bounds on the second fundamental form of the supporting hypersurfaces, together with an application of the $L^{\infty}$ case of [26, Proposition 1.1] proves

Corollary 8.3. $\tau \in C_{\text {Loc }}^{1,1}$.

We leave to the reader to formulate the obvious analogs of Theorem 1.4 and Corollary 8.3 for past complete flat regular domains $E^{-}(\Lambda)$ which hold in term of the reverse cosmological time $\widehat{\tau}: E^{-}(\Lambda) \rightarrow(0,+\infty)$.

9. CMC time functions in flat MGHC spacetimes. Let $(M, g)$ be an $n$ dimensional flat MGHC spacetime. We first consider the case where $(M, g)$ is not past complete. Then Theorem 6.7 states that $(M, g)$ is the quotient of a future complete regular domain $E^{+}(\Lambda) \subset \mathbb{R}^{1, n-1}$ by a torsion-free discrete subgroup $\Gamma$ of $\operatorname{Isom}\left(\mathbb{R}^{1, n-1}\right)$. Let $\tau: E^{+}(\Lambda) \rightarrow(0,+\infty)$ be the cosmological time of $E^{+}(\Lambda)$. It follows from Theorem 2.3 and its proof, see [5, Proposition 2.2], that for every $a \in$ $(0,+\infty)$, the level set $S_{a}=\tau^{-1}(a)$ is a closed strictly achronal edgeless hypersurface in $E^{+}(\Lambda)$. Moreover, $\tau$ is obviously invariant under every element of $\operatorname{Isom}\left(\mathbb{R}^{1, n-1}\right)$ preserving $E^{+}(\Lambda)$. Hence, for every $a \in(0,+\infty)$, the projection $\Sigma_{a}$ of $S_{a}$ in $M \equiv$ $\Gamma \backslash E^{+}(\Lambda)$ is a closed strictly achronal edgeless hypersurface in $M$. Since $M$ is globally hyperbolic with compact Cauchy hypersurfaces, this implies that $\Sigma_{a}$ is a compact strictly achronal hypersurface in $M$, and thus is a topological Cauchy hypersurface in $M$. Theorem 1.4 implies that, for every $a \in(0,+\infty), \Sigma_{a}$ has generalized mean curvature bounded from below by $-1 / a$, and bounded from above by $-1 /((n-1) a)$. Let $\left(a_{m}\right)_{m \in \mathbb{N}}$ be a decreasing sequence of positive real numbers such that $a_{m} \rightarrow 0$ when $m \rightarrow+\infty$, and $\left(b_{m}\right)_{m \in \mathbb{N}}$ be a increasing sequence of positive real numbers such that $b_{m} \rightarrow+\infty$ when $m \rightarrow+\infty$. Observe that $\left(\Sigma_{a_{m}}\right)_{m \in \mathbb{N}}$ is a sequence of past asymptotic $\alpha$-barrier in $M$ for $\alpha=-\infty$ (indeed $-\infty<-1 / a_{m}<-1 /\left((n-1) a_{m}\right)$ for every $m$, and since $-1 /\left((n-1) a_{m}\right) \rightarrow-\infty$ when $\left.m \rightarrow \infty\right)$, and $\left(\Sigma_{b_{m}}\right)_{m \in \mathbb{N}}$ is a sequence of future asymptotic $\beta$-barrier in $M$ for $\beta=0$ (indeed $-1 / b_{m}<-1 /\left((n-1) b_{m}\right)<0$ ) for every $m$, and since $-1 / b_{m} \rightarrow 0$ when $\left.m \rightarrow \infty\right)$. Hence Theorem 4.3 implies that $M$ admits a globally defined CMC time function $\tau_{c m c}: M \rightarrow(-\infty, 0)$.

Next, we prove that $\tau$ and $\tau_{c m c}$ are comparable. It follows from Theorem 1.4 that for every $a>0$, the pair of hypersurfaces $\left(\Sigma_{a /(n-1)}, \Sigma_{a}\right)$ is a pair of $-1 / a$-barriers. Hence, Theorem 4.9 and remark 4.8 imply that the hypersurface $\tau_{c m c}^{-1}(-1 / a)$ is in the future of $\Sigma_{a /(n-1)}=\tau^{-1}(a /(n-1))$ and in the past of $\Sigma_{a}=\tau^{-1}(a)$. Equivalently, one has

$$
\tau \leq-\frac{1}{\tau_{c m c}} \leq(n-1) \tau
$$


The case where $(M, g)$ is future incomplete is similar (except that $(M, g)$ is the quotient of a past complete flat regular domain $E^{-}(\Lambda)$, and that one has to consider the reverse cosmological time of $\left.E^{-}(\Lambda)\right)$.

Finally, let us consider the case where $(M, g)$ is causally complete. Then Theorem 6.7 states that up to a finite covering $(M, g)$ is a quotient of $\mathbb{R}^{1, n-1}$ by a commutative subgroup $\Gamma$ of $\operatorname{Isom}\left(\mathbb{R}^{1, n-1}\right)$ generated by $n-1$ spacelike linearly independant translations $t_{\overrightarrow{u_{1}}}, \ldots, t_{\overrightarrow{u_{n}}}$. Let $\vec{v}$ be any (say future-directed) timelike vector. Then, for every $t \in \mathbb{R}$, the affine plane $P_{t}:=t \cdot \vec{v}+\mathbb{R} \cdot \overrightarrow{u_{1}}+\cdots+\mathbb{R} \cdot \overrightarrow{u_{n}}$ is $\Gamma$-invariant. Hence it induces a totally geodesic spacelike hypersurface $\Sigma_{t}:=\Gamma \backslash P_{t}$ in $M \simeq \Gamma \backslash \mathbb{R}^{1, n-1}$. The family of hypersurfaces $\left(\Sigma_{t}\right)_{t \in \mathbb{R}}$ is a foliation of $M$ whose leaves are totally geodesic (in particular, CMC) spacelike hypersurfaces.

In order to complete the proof of Theorem 1.4, we only need to prove that in the case where $(M, g)$ is causally complete, every CMC Cauchy hypersurface $\Sigma$ in $M$ is a leaf of the totally geodesic foliation $\left(\Sigma_{t}\right)_{t \in \mathbb{R}}$ constructed above. Indeed, let $t^{-}=\inf \left\{t\right.$ such that $\left.\Sigma \cap \Sigma_{t} \neq \emptyset\right\}$ and $t^{+}=\sup \left\{t\right.$ such that $\left.\Sigma \cap \Sigma_{t} \neq \emptyset\right\}$. Then, $\Sigma$ is tangent to $\Sigma_{t^{-}}$at some point and is in the future of $\Sigma_{t^{-}}$. Hence, the maximum principle (Proposition 4.4) implies that the mean curvature of $\Sigma$ is smaller or equal to those of $\Sigma_{t^{-}}$, i.e. is non-positive. Similarly, $\Sigma$ is tangent to $\Sigma_{t^{+}}$at some point and is in the past of $\Sigma_{t^{+}}$, so by the maximum principle, the mean curvature of $\Sigma$ is non-negative. So, we know that the mean curvature of $\Sigma$ is equal to 0 . And now, we use the equality case of the maximum principle (see, e.g., [6, Theorem 3.6]): if $S$ and $S^{\prime}$ are two CMC Cauchy hypersurfaces with the same mean curvature, which are tangent at some point, and such that $S^{\prime}$ is in the future of $S$, then $S=S^{\prime}$. This shows that $\Sigma=\Sigma_{t^{-}}=\Sigma_{t^{+}}$; in particular, $\Sigma$ is a leaf of the totally geodesic foliation $\left(\Sigma_{t}\right)_{t \in \mathbb{R}}$.

10. Description of anti-de Sitter MGHC spacetimes. We now start our investigation of anti-de Sitter spacetimes. Our goal is to prove Theorem 1.7. According to Theorem 4.3, this reduces to finding two sequences of asymptotic barriers. These sequences of barriers will be provided by the level sets of the cosmological time function. Thus, we essentially need to prove curvature estimates for the level sets of the cosmological time function of any anti-de Sitter MGHC spacetime. A key point is that every MGHC spacetime with constant curvature -1 is isometric to the quotient of a certain open domain in the anti-de Sitter space $\mathrm{AdS}_{n}$ by a discrete subgroup of Isom $\left(\operatorname{AdS}_{n}\right)$. A consequence is that studying the cosmological time functions of antide Sitter MGHC spacetimes amounts to studying the cosmological time functions of certain open domains in $\mathrm{AdS}_{n}$. These domains are called AdS regular domains.

We will proceed as follows. In the present section, we define AdS regular domains, using the conformal structure of the anti-de Sitter space. We shall also give two characterizations of AdS regular domains, using the Klein model of the anti-de Sitter space. In section 11, we shall study the cosmological time and the boundary of AdS regular domains. The desired estimates on the curvature of the level sets of the cosmological time of AdS regular domains will be obtained in section 12. Theorem 1.7 follows easily from these estimates and from Theorem 4.3.

10.1. The linear model $\mathbf{A d S}_{n}$ of the anti-de Sitter space. For $n \geq 2$, let $\left(x_{1}, \ldots, x_{n+1}\right)$ be the standard coordinates on $\mathbb{R}^{n+1}$, and consider the quadratic form $Q_{2, n-1}=-x_{1}^{2}-x_{2}^{2}+x_{3}^{2}+\cdots+x_{n+1}^{2}$. The linear model $A d S_{n}$ of the $n$-dimensional anti-de Sitter space is the quadric $\left(Q_{2, n-1}=-1\right)$, endowed with the lorentzian metric induced by $Q_{2, n-1}$. 
It is very easy to see that $\operatorname{AdS}_{n}$ is diffeomorphic to $\mathbb{S}^{1} \times \mathbb{D}^{n-1}$. The geodesics of $\mathrm{AdS}_{n}$ are connected components of the intersections of $\mathrm{AdS}_{n}$ with the linear 2planes in $\mathbb{R}^{n+1}$. Similarly, the totally geodesic subspaces of dimension $k$ in $\operatorname{AdS}_{n}$ are connected components of the intersections of $A d S_{n}$ with the linear subspaces of dimension $(k+1)$ in $\mathbb{R}^{n+1}$.

A nice feature of the anti-de Sitter space is its simple conformal structure.

Proposition 10.1. The anti-de Sitter space $A d S_{n}$ is conformally equivalent to $\left(\mathbb{S}^{1} \times \mathbb{D}^{n-1},-d t^{2}+d s^{2}\right)$, where $d t^{2}$ is the standard riemannian metric on $\mathbb{S}^{1}=\mathbb{R} / 2 \pi \mathbb{Z}$, where $d s^{2}$ is the standard metric (of curvature +1 ) on the sphere $\mathbb{S}^{n-1}$ and $\mathbb{D}^{n-1}$ is the open upper hemisphere of $\mathbb{S}^{n-1}$.

Moreover, one can attach a Penrose boundary $\partial \widetilde{A d S}$ to $\widetilde{A d S}$ such that $\widetilde{A d S}_{n} \cup$ $\partial \widetilde{A d S_{n}}$ is conformally equivalent to $\left(\mathbb{S}^{1} \times \overline{\mathbb{D}^{n-1}},-d t^{2}+d s^{2}\right)$, where $\overline{\mathbb{D}^{n-1}}$ is the closed upper hemisphere of $\mathbb{S}^{n-1}$.

Proposition 10.1 shows in particular that $\mathrm{AdS}_{n}$ contains many closed causal curves. One can overcome this difficulty by considering the universal covering $\widetilde{\operatorname{AdS}}_{n}$ of $\mathrm{AdS}_{n}$. It follows from Proposition 10.1 that $\widetilde{\mathrm{AdS}}_{n}$ is conformally equivalent to $\left(\mathbb{R} \times \mathbb{D}^{n-1},-d t^{2}+d s^{2}\right)$, and admits a Penrose boundary $\partial \widetilde{\mathrm{AdS}}_{n}$ such that $\widetilde{\operatorname{AdS}}_{n} \cup \partial \widetilde{\operatorname{AdS}}_{n}$ is conformally equivalent to $\left(\mathbb{R} \times \overline{\mathbb{D}^{n-1}},-d t^{2}+d s^{2}\right)$. In particular, $\widetilde{\operatorname{AdS}}_{n}$ and $\widetilde{\operatorname{AdS}}_{n} \cup \partial \widetilde{\mathrm{AdS}}_{n}$ are strongly causal.

Proof of Proposition 10.1. See e.g. [12, $\S 4]$ or [14, Proposition 4.16].

10.2. AdS regular domains as subsets of $\mathbf{A d S}_{n}$. In this paragraph, we will use the conformal completion $\mathrm{AdS}_{n} \cup \partial \mathrm{AdS}_{n}$ of $\mathrm{AdS}_{n}$ to define the notion of $A d S$ regular domain. Let us start by a remark.

REMARK 10.2. A subset $\widetilde{\Lambda}$ of $\partial \widetilde{\mathrm{AdS}}_{n} \approx\left(\mathbb{R} \times \mathbb{S}^{n-2},-d t^{2}+d s^{2}\right)$ is achronal if and only if it is the graph of a 1-Lipschitz function $f: \Lambda_{0} \rightarrow \mathbb{R}$ where $\Lambda_{0}$ is a subset of $\mathbb{S}^{n-2}$ (endowed with its canonical distance, induced by the metric $d s^{2}$ of curvature 1 ). In particular, the achronal closed topological hypersurfaces in $\partial \widetilde{\mathrm{AdS}}_{n}$ are exactly the graphs of the 1-Lipschitz functions $f: \mathbb{S}^{n-2} \rightarrow \mathbb{R}$. In particular, every closed achronal hypersurface in $\partial \widetilde{\mathrm{AdS}}_{n}$ is a topological $(n-2)$-sphere.

Let $\widetilde{\Lambda}$ be a closed achronal subset of $\partial \widetilde{\mathrm{AdS}}_{n}$, and $\Lambda$ be the projection of $\widetilde{\Lambda}$ in $\partial \operatorname{AdS}_{n}$. We denote by $\widetilde{E}(\widetilde{\Lambda})$ the invisible domain of $\widetilde{\Lambda}$ in $\widetilde{\operatorname{AdS}}_{n} \cup \partial \widetilde{\operatorname{AdS}}_{n}$, that is,

$$
\widetilde{E}(\widetilde{\Lambda})=\left(\widetilde{\operatorname{AdS}}_{n} \cup \partial \widetilde{\operatorname{AdS}}_{n}\right) \backslash\left(J^{-}(\widetilde{\Lambda}) \cup J^{+}(\widetilde{\Lambda})\right)
$$

where $J^{-}(\widetilde{\Lambda})$ and $J^{+}(\widetilde{\Lambda})$ are the causal past and the causal future of $\widetilde{\Lambda}$ in $\widetilde{\mathrm{AdS}}_{n} \cup$ $\partial \widetilde{\mathrm{AdS}}_{n}=\left(\mathbb{R} \times \overline{\mathbb{D}}^{n-1},-d t^{2}+d s^{2}\right)$. We denote by $\mathrm{Cl}(\widetilde{E}(\widetilde{\Lambda}))$ the closure of $\widetilde{E}(\widetilde{\Lambda})$ in $\widetilde{\operatorname{AdS}}_{n} \cup \partial \widetilde{\operatorname{AdS}}_{n}$. We denote by $E(\Lambda)$ the projection of $\widetilde{E}(\widetilde{\Lambda})$ in $\operatorname{AdS}_{n} \cup \partial \operatorname{AdS}_{n}$ (clearly, $E(\Lambda)$ only depends on $\Lambda$, not on $\widetilde{\Lambda})$.

Definition 10.3. A $n$-dimensional AdS regular domain is a domain of the form $E(\Lambda)$ where $\Lambda$ is the projection in $\partial \mathrm{AdS}_{n}$ of an achronal topological $(n-2)$-sphere $\widetilde{\Lambda} \subset \partial \operatorname{AdS}_{n}$.

We will see later that regular domains satisfy several "convexity properties" (geodesic convexity, convexity in a projective space). The first property of this kind concerns the causal structure. 
REMARK 10.4. For every closed achronal set $\widetilde{\Lambda}$ in $\partial \widetilde{\operatorname{AdS}}_{n}$, the invisible domain $\widetilde{E}(\widetilde{\Lambda})$ is a causally convex subset of $\widetilde{A d S}_{n} \cup \partial \widetilde{\mathrm{AdS}}_{n}$ : if $p, q \in \widetilde{E}(\widetilde{\Lambda})$ then $J^{+}(p) \cap$ $J^{-}(q) \subset \widetilde{E}(\widetilde{\Lambda})$, where $J^{+}(p)$ and $J^{-}(q)$ are the causal past and future of $p$ and $q$ in $\widehat{\operatorname{AdS}}_{n} \cup \partial \widehat{\operatorname{AdS}}_{n}$. This is an immediate consequence of the definitions.

The following remark is a key point for understanding the geometry of AdS regular domains.

REMARK 10.5. Let $\widetilde{\Lambda}$ be a closed achronal subset of $\partial \widetilde{\operatorname{AdS}}_{n}$. Recall that $\widetilde{\Lambda}$ is the graph of a 1-Lipschitz function $f: \Lambda_{0} \rightarrow \mathbb{R}$ where $\Lambda_{0}$ is a closed subset of $\mathbb{S}^{n-2}$ (remark 10.2). Define two functions $f^{-}, f^{+}: \overline{\mathbb{D}}^{n-1} \rightarrow \mathbb{R}$ as follows:

$$
\begin{aligned}
& f^{-}(p)=\operatorname{Sup}_{q \in \Lambda_{0}}\{f(q)-d(p, q)\}, \\
& f^{+}(p)=\operatorname{Inf}_{q \in \Lambda_{0}}\{f(q)+d(p, q)\},
\end{aligned}
$$

where $d$ is the distance induced by $d s^{2}$ on $\overline{\mathbb{D}}^{n-1}$. It is easy to check that

$$
\widetilde{E}(\widetilde{\Lambda})=\left\{(t, p) \in \mathbb{R} \times \overline{\mathbb{D}}^{n-1} \mid f^{-}(p)<p<f^{+}(p)\right\} .
$$

Corollary 10.6. For every (non-empty) closed achronal set $\widetilde{\Lambda} \subset \partial \widetilde{A d S_{n}}$, the projection of $\widetilde{E}(\widetilde{\Lambda})$ on $E(\Lambda)$ is one-to-one.

Proof. We use the notations introduced in remark 10.5. For every $p \in \overline{\mathbb{D}}^{n-1}$, there exists a point $q \in \mathbb{S}^{n-2}=\partial \overline{\mathbb{D}}^{n-1}$ such that $d(p, q) \leq \pi / 2$. Hence, for every $p \in \overline{\mathbb{D}}^{n-1}$, we have $f^{+}(p)-f^{-}(p) \leq \pi$. Hence $\widetilde{E}(\widetilde{\Lambda})$ is included in the set $E=\{(t, p) \in \mathbb{R} \times$ $\overline{\mathbb{D}^{n-1}}$ such that $\left.f^{-}(p)<t<f^{-}(p)+\pi\right\}$. The projection of $\widetilde{\operatorname{AdS}}_{n} \cup \partial \widetilde{\mathrm{AdS}}_{n}=\mathbb{R} \times \overline{\mathbb{D}}^{n-1}$ on $\operatorname{AdS}_{n} \cup \partial \operatorname{AdS}_{n}=(\mathbb{R} / 2 \pi \mathbb{Z}) \times \overline{\mathbb{D}}^{n-1}$ is obviously one-to-one in restriction to $E$. $\mathrm{u}$

CoRollary 10.7. For every achronal topological $(n-2)$-sphere $\widetilde{\Lambda} \subset \partial \widetilde{A d S_{n}}$,

1. $\widetilde{E}(\widetilde{\Lambda})$ is disjoint from $\partial \widetilde{A d S}_{n}$ (i.e. it is contained in $\widetilde{A d S}_{n}$ );

2. $C l(\widetilde{E}(\widetilde{\Lambda})) \cap \partial \widetilde{A d S}_{n}=\widetilde{\Lambda}$.

Proof. We use the notations introduced in remark 10.5. Since $\widetilde{\Lambda}$ is a topological $(n-2)$-sphere, the set $\Lambda_{0}$ is the whole sphere $\mathbb{S}^{n-2}$. Now observe that, for every $p \in \mathbb{S}^{n-2}=\Lambda_{0}$, one has $f^{-}(p)=f^{+}(p)=p$. Finally, recall that $(t, p) \in \widetilde{E}(\widetilde{\Lambda})$ (resp. $(t, p) \in \mathrm{Cl}(\widetilde{E}(\widetilde{\Lambda})))$ if and only if $f^{-}(p)<t<f^{+}(p)$ (resp. $\left.f^{-}(p) \leq t \leq f^{+}(p)\right)$. The corollary follows.

Definition 10.8. Let $\Lambda_{0}$ be a closed subset of $\mathbb{S}^{n-2}$, let $f: \Lambda_{0} \rightarrow \mathbb{R}$ be a 1Lipschitz function, and $\widetilde{\Lambda} \subset \partial \widetilde{\mathrm{AdS}}_{n}$ be the graph of $f$. The achronal set $\widetilde{\Lambda}$ is said to be pure lightlike if $\Lambda_{0}$ contains two antipodal points $p_{0}$ and $-p_{0}$ on the sphere such that $f\left(p_{0}\right)=f\left(-p_{0}\right)+\pi$.

Lemma 10.9. If $\widetilde{\Lambda}$ is pure lightlike, then $\widetilde{E}(\widetilde{\Lambda})$ is empty.

Proof. If $f: \Lambda_{0} \rightarrow \mathbb{R}$ is 1-Lipschitz, and if there exists two antipodal points $p_{0},-p_{0} \in \Lambda_{0}$ such that $f\left(p_{0}\right)=f\left(-p_{0}\right)+\pi$, then it is easy to show that, for every element $p$ of $\overline{\mathbb{D}}^{n-1}$, we have $f_{-}(p)=f_{+}(p)=f\left(-p_{0}\right)+d\left(-p_{0}, p\right)=f\left(p_{0}\right)-d\left(p_{0}, p\right)$. The lemma follows. 
10.3. The Klein model $\mathbb{A D S}_{n}$ of the anti-de Sitter space. We now consider the quotient $\mathbb{S}\left(\mathbb{R}^{n+1}\right)$ of $\mathbb{R}^{n+1} \backslash\{0\}$ by positive homotheties. In other words, $\mathbb{S}\left(\mathbb{R}^{n+1}\right)$ is the double covering of the projective space $\mathbb{P}\left(\mathbb{R}^{n+1}\right)$. We denote by $\pi$ the projection of $\mathbb{R}^{n+1}$ on $\mathbb{S}\left(\mathbb{R}^{n+1}\right)$. The projection $\pi$ is one-to-one in restriction to $\operatorname{AdS}_{n}=\left(Q_{2, n-1}=\right.$ $-1)$. The Klein model $\mathbb{A D S}_{n}$ of the anti-de Sitter space is the projection of $\mathrm{AdS}_{n}$ in $\mathbb{S}\left(\mathbb{R}^{n+1}\right)$, endowed with the induced lorentzian metric.

Observe that $\mathbb{A D S}_{n}$ is also the projection of the open domain of $\mathbb{R}^{n+1}$ defined by the inequality $\left(Q_{2, n-1}<0\right)$. It follows that the topological boundary of $\mathbb{A D S}_{n}$ in $\mathbb{S}\left(\mathbb{R}^{n+1}\right)$ is the projection of the quadric $\left(Q_{2, n-1}=0\right)$; we will denote this boundary by $\partial \mathbb{A D S}_{n}$. By construction, the projection $\pi$ defines an isometry between $\mathrm{AdS}_{n}$ and $\mathbb{A D S}_{n}$; one can easily verify that this isometry can be continued to define a canonical homeomorphism between $\operatorname{AdS}_{n} \cup \partial \mathrm{AdS}_{n}$ and $\mathbb{A D S}_{n} \cup \partial \mathbb{A D S}_{n}$.

For every linear subspace $F$ of dimension $k+1$ in $\mathbb{R}^{n+1}$, we denote by $\mathbb{S}(F)=\pi(F)$ the corresponding projective subspace of dimension $k$ in $\mathbb{S}\left(\mathbb{R}^{n+1}\right)$. The geodesics of $\mathbb{A D S}_{n}$ are connected components of the intersections of $\mathbb{A D S}_{n}$ with the projective lines $\mathbb{S}(F)$ of $\mathbb{S}\left(\mathbb{R}^{n+1}\right)$. More generally, the totally geodesic subspaces of dimension $k$ in $\mathbb{A D S}_{n}$ are connected components of the intersections of $\mathbb{A D S}_{n}$ with the projective subspaces $\mathbb{S}(F)$ of dimension $k$ of $\mathbb{S}\left(\mathbb{R}^{n+1}\right)$.

DeFinition 10.10. An affine domain of $\mathbb{A D S}_{n}$ is a connected component $U$ of $\mathbb{A D S}_{n} \backslash \mathbb{S}(F)$, where $\mathbb{S}(F)$ is a projective hyperplane of $\mathbb{S}\left(\mathbb{R}^{n+1}\right)$ such that $\mathbb{S}(F) \cap \mathbb{A D} \mathbb{S}_{n}$ is a spacelike (totally geodesic) hypersurface. Let $V$ be the connected component of $\mathbb{S}\left(\mathbb{R}^{n+1}\right) \backslash \mathbb{S}(F)$ containing $U$. The boundary $\partial U \subset \partial \mathbb{A D} \mathbb{S}_{n}$ of $U$ in $V$ is called the affine boundary of $U$.

REMARK 10.11. Affine domains can be visualized in $\mathbb{R}^{n}$. Indeed, let $U$ be an affine domain in $\mathbb{A D S}_{n}$. By definition, there exists a a projective hyperplane $\mathbb{S}(F)$ in $\mathbb{S}\left(\mathbb{R}^{n+1}\right)$ such that the hypersurface $\mathbb{S}(F) \cap \mathbb{A D} \mathbb{S}_{n}$ is spacelike, and such that $U$ is one of the two connected components of $\mathbb{A D S}_{n} \backslash \mathbb{S}(F)$. We denote by $V$ the connected component of $\mathbb{S}\left(\mathbb{R}^{n+1}\right)$ containing $U$. Up to composition by an element of the isometry group $S O_{0}(2, n-1)$ of $Q_{2, n-1}$, we can assume that $\mathbb{S}(F)$ is the projection of the hyperplane $\left(x_{1}=0\right)$ in $\mathbb{R}^{n+1}$ and $V$ is the projection of the region $\left(x_{1}>0\right)$ in $\mathbb{R}^{n+1}$. The map

$$
\left(x_{1}, x_{2}, \ldots, x_{n+1}\right) \mapsto\left(u_{1}, \ldots, u_{n}\right):=\left(\frac{x_{2}}{x_{1}}, \frac{x_{3}}{x_{1}}, \ldots, \frac{x_{n+1}}{x_{1}}\right)
$$

induces a diffeomorphism between $V$ and $\mathbb{R}^{n}$. In the coordinates $\left(u_{1}, \ldots, u_{n}\right)$, the image of the affine domain $U$ corresponds to the region $\left(-u_{1}^{2}+u_{2}^{2}+\cdots+u_{n}^{2}<1\right)$. The affine boundary $\partial U$ of $U$ corresponds to the hyperboloid $\left(-u_{1}^{2}+u_{2}^{2}=+\cdots+u_{n}^{2}=1\right)$. The intersection of $U$ with the totally geodesic subspaces of $\mathbb{A D S}_{n}$ correspond to the intersections of the region $\left(-u_{1}^{2}+u_{2}^{2}+\cdots+u_{n}^{2}<1\right)$ with the affine subspaces of $\mathbb{R}^{n}$.

10.4. AdS regular domains as subsets of $\mathbb{A D S}_{n}$. The canonical diffeomorphism between $\mathrm{AdS}_{n} \cup \partial \mathrm{AdS}_{n}$ and $\mathbb{A D S}_{n} \cup \partial \mathbb{A D S}_{n}$ allows us to see AdS regular domains as subsets of $\mathbb{A D S}_{n}$. Nevertheless, it would be much more interesting to characterize AdS regular domains directly as subsets of $\mathbb{A D S}_{n}$ without using the identification of $\mathbb{A D S}_{n} \cup \partial \mathbb{A D S}_{n}$ with $\mathrm{AdS}_{n} \cup \partial \mathrm{AdS}_{n}$; this is the purpose of the present section. We start by stating the following lemma.

LEMMA 10.12. Let $\Lambda \subset \partial A d S_{n}$ be the projection of a closed achronal subset of $\partial \widetilde{A d S}_{n}$ which is not pure lightlike. We see $\Lambda$ and $E(\Lambda)$ in $\mathbb{A D S}_{n} \cup \partial \mathbb{A D S}_{n}$. Then 
$\Lambda$ and $E(\Lambda)$ are contained in the union $U \cup \partial U$ of an affine domain and its affine boundary.

Proof. See [12, Lemma 8.27].

Lemma 10.12 implies, in particular, that every AdS regular domain is contained in an affine domain $U$ of $\mathbb{A D S}_{n}$. This allows one to visualize AdS regular domains as subsets of $\mathbb{R}^{n}$ (see remark 10.11).

We will now use the pseudo-scalar product $\langle\cdot \mid \cdot\rangle$ associated with the quadratic form $Q_{2, n-1}$. It is important to note that, although the real number $\langle x \mid y\rangle$ is welldefined only for $x, y \in \mathbb{R}^{n+1}$, the sign of $\langle x \mid y\rangle$ is well-defined for $x, y \in \mathbb{S}\left(\mathbb{R}^{n+1}\right)$. The following lemma is easy but fundamental.

LEMma 10.13. Let $U$ be an affine domain in $\mathbb{A D S}_{n}$ and $\partial U \subset \partial \mathbb{A D S}_{n}$ be its affine boundary. Let $x$ be be a point in $\partial U$, and $y$ be a point in $U \cup \partial U$. There exists a causal (resp. timelike) curve joining $x$ to $y$ in $U \cup \partial U$ if and only if $\langle x \mid y\rangle \geq 0$ (resp. $\langle x \mid y\rangle>0$ ).

Proof. See e.g. [12, Proposition 5.10] or [14, Proposition 4.19].

Putting together the definition of the invisible domain $E(\Lambda)$ of a set $\Lambda \subset \partial \operatorname{AdS}_{n}$ and Lemma 10.13, one easily proves the following.

Proposition 10.14. Let $\Lambda \subset \partial A d S_{n}$ be the projection of a closed achronal subset of $\partial \widetilde{A d S_{n}}$ which is not pure lightlike. If we see $\Lambda$ and $E(\Lambda)$ in the Klein model $\mathbb{A D S}_{n} \cup \partial \mathbb{A D S}_{n}$, then

$$
\left.E(\Lambda)=\left\{y \in \mathbb{A D S}_{n} \cup \partial \mathbb{A D S}_{n} \text { such that }\langle y \mid x\rangle<0 \text { for every } x \in \Lambda\right\}\right) .
$$

REMARK 10.15. A nice (and important) corollary of this Proposition is that the invisible domain $E(\Lambda)$ associated with a set $\Lambda$ is always geodesically convex, i.e. any geodesic joining two points in $E(\Lambda)$ is contained in $E(\Lambda)$.

Proposition 10.14 provides a characterization of the AdS regular domain associated to the projection of an achronal topological $(n-2)$-sphere of $\partial \widetilde{\mathrm{AdS}}_{n}$. In order to obtain a complete definition of AdS regular domains in $\mathbb{A D S}_{n}$, it remains to identify the subsets of $\partial \mathrm{ADS}_{n}$ which correspond to the projections of achronal topological spheres $\partial \widetilde{\mathrm{AdS}}_{n}$. This is the purpose of the following proposition, which easily follows from Lemma 10.13.

Proposition 10.16. For $\Lambda \subset \partial \mathbb{A D S}_{n}$, the following assertions are equivalent.

1. when we see $\Lambda$ as a subset of $\partial A d S_{n}$, it is the projection of an achronal subset of $\partial \widetilde{A d S}_{n}$,

2. $\langle x \mid y\rangle$ is non-positive for every $x, y \in \Lambda$.

Moreover, if $\Lambda$ satifies these assertions, $\Lambda$ is purely lightlike if and only it contains two antipodal points of $\mathbb{S}\left(\mathbb{R}^{n+1}\right)$.

Finally, we will give another characterization of the AdS regular domains, using the duality for convex subsets of $\mathbb{S}\left(\mathbb{R}^{n}\right)$.

Let us first recall some standard definitions. A convex cone $J$ of $\mathbb{R}^{n+1}$ is a convex subset stable by positive homotheties. A convex cone $J \subset \mathbb{R}^{n+1}$ is said to be proper if it is nonempty, and if its closure $\bar{J}$ does not contain a complete affine line. A convex subset $C$ of $\mathbb{S}\left(\mathbb{R}^{n+1}\right)$ is the projection of a convex cone $J(C)$ of $\mathbb{R}^{n}$; it is proper if 
$J(C)$ can be chosen proper. Now, for any convex cone $J \subset \mathbb{R}^{n+1}$, one can define the dual convex cone $J^{*}$ of $J$,

$$
J^{*}=\left\{x \in \mathbb{R}^{n+1} \text { such that }\langle x \mid y\rangle<0 \text { for all } y \in \bar{J} \backslash\{0\}\right\}
$$

This allows one to associate a dual convex set $C^{*} \subset \mathbb{S}\left(\mathbb{R}^{n+1}\right)$ to any convex set $C \subset \mathbb{S}\left(\mathbb{R}^{n+1}\right)$. Note that $J^{* *}=J$ and $C^{* *}=C$.

Using this duality, Proposition 10.14 can be reformulated as follows.

Proposition 10.17. Let $\Lambda \subset \partial A d S_{n}$ be the projection of a closed achronal subset of $\partial \widetilde{A d S}_{n}$. We see $\Lambda$ and $E(\Lambda)$ in $\mathbb{A D S}_{n} \cup \partial \mathbb{A D S}_{n}$. Then the domain $E(\Lambda)$ is the dual of the convex hull of $\Lambda$ in $\mathbb{S}\left(\mathbb{R}^{n+1}\right)$.

In particular, AdS regular domains are the duals of the convex hulls of the achronal topological $(n-2)$-sphere in $\partial \mathbb{A D S}_{n}$.

10.5. Cosmological time and horizon of AdS regular domains. The link between MGHC spacetimes with constant curvature -1 and AdS regular domains is made explicit by the following theorem.

THEOREM 10.18. Every $n$-dimensional MGHC spacetime with constant curvature -1 is isometric to the quotient of a regular domain in $A d S_{n}$ by a torsion-free discrete subgroup of $\operatorname{Isom}\left(A d S_{n}\right)$.

This result was proved by Mess in his celebrated preprint [40] (Mess only deals with the case where $n=3$, but his arguments also apply in higher dimension). For the reader's convenience, we shall recall the main steps of the proof (see [12, Corollary 11.2] for more details).

Sketch of proof of Theorem 10.18. Let $(M, g)$ be a MGHC spacetime of dimension $n$ with constant curvature -1 . As explained in section 5 , the theory of $(G, X)$ structures provides us with a locally isometric developing map $d: \widetilde{M} \rightarrow \operatorname{AdS}_{n}$ and a holonomy representation $\rho: \pi_{1}(M) \rightarrow \operatorname{Isom}\left(\operatorname{AdS}_{n}\right)$. Pick a Cauchy hypersurface $\Sigma$ in $M$, and a lift $\widetilde{\Sigma}$ of $\Sigma$ in $\widetilde{M}$. Then $S:=d(\widetilde{\Sigma})$ is an immersed complete spacelike hypersurface in $\mathrm{AdS}_{n}$. One can prove that such a hypersurface is automatically properly embedded and corresponds to the graph of a 1-Lipschitz function $f: \mathbb{D}^{2} \rightarrow \mathbb{S}^{1}$ in the conformal model $\left(\mathbb{S}^{1} \times \mathbb{D}^{2},-d t^{2}+d s^{2}\right)$. Such a function extends to a 1-Lipschitz function $\bar{f}$ defined on the closed disc $\overline{\mathbb{D}}^{2}$. This shows that the boundary $\partial S$ of $S$ in $\mathrm{AdS}_{n} \cup \partial \mathrm{AdS}_{n}$ is an achronal curve contained in $\partial \mathrm{AdS}_{n}$.

On the one hand, it is easy to see that the Cauchy development $D(S)$ coincides with the invisible domain $E(\partial S)$ (this essentially relies on the fact that $S \cup \partial S$ is the graph of a 1-Lipschitz function, hence an achronal set in $\widetilde{\operatorname{AdS}}_{n}$ ). In particular, this shows that $D(S)$ is an AdS regular domain.

On the other hand, one can prove that $M$ is isometric to the quotient $\Gamma \backslash D(S)$, where $\Gamma:=\rho\left(\pi_{1}(M)\right)$. Indeed, recall that $S=d(\widetilde{\Sigma})$ is a properly embedded hypersurface. This shows that the group $\Gamma$ acts freely and properly discontinuously on $S=d(\widetilde{\Sigma})$. It is easy to deduce that $\Gamma$ acts freely and properly discontinuously on the Cauchy development $D(S)$. Hence the quotient $\Gamma \backslash D(S)$ is a globally hyperbolic spacetime. Now, observe that $d(\widetilde{M})$ is necessarily contained in $D(S)$ since $\widetilde{\Sigma}$ is a Cauchy hypersurface in $\widetilde{M}$. Moreover, since $S$ is embedded in $M$, the developing map $d$ is one-to-one in restriction to $\widetilde{\Sigma}$. It follows that $d$ is one-to-one on the Cauchy development of $\widetilde{\Sigma}$, i.e. on $\widetilde{M}$. Hence the developing map $d$ induces an isometric embedding 
of $M$ in the $\Gamma \backslash D(S)$. Since $M$ is maximal, this embedding must be onto, and thus, $M$ is isometric to the quotient $\Gamma \backslash D(S)$.

11. Cosmological time and horizons of AdS regular domains. Throughout this section, we consider an achronal topological $(n-2)$-sphere $\Lambda$ in $\partial \mathrm{AdS}_{n}$, and the associated AdS regular domain $E(\Lambda)$.

\subsection{The cosmological time function.}

Proposition 11.1. The AdS regular domain $E(\Lambda)$ has regular cosmological time.

Proof. We recall that $\Lambda$ is, by definition, the projection of an achronal topological sphere $\widetilde{\Lambda} \subset \partial \widetilde{\mathrm{AdS}}_{n}$, and that $E(\Lambda)$ is the projection of the invisible domain $\widetilde{E}(\widetilde{\Lambda})$ of $\widetilde{\Lambda}$ in $\widetilde{\operatorname{AdS}}_{n} \cup \partial \widetilde{\operatorname{AdS}}_{n}$. We will prove that $\widetilde{E}(\widetilde{\Lambda})$ has regular cosmological time. Since the projection of $\widetilde{E}(\widetilde{\Lambda})$ on $E(\Lambda)$ is one-to-one (corollary 10.6), this will imply that $E(\Lambda)$ also has regular cosmological time. We denote by $\widetilde{\tau}$ the cosmological time of $\widetilde{E}(\widetilde{\Lambda})$.

Let $x$ be a point in $\widetilde{E}(\widetilde{\Lambda})$. On the one hand, corollary 10.7 states that $\operatorname{Cl}(\widetilde{E}(\widetilde{\Lambda}))$ is a compact subset of $\widetilde{\operatorname{AdS}}_{n} \cup \partial \widehat{\operatorname{AdS}}_{n}$, and that $\mathrm{Cl}(\widetilde{E}(\widetilde{\Lambda})) \cap \partial \widetilde{\operatorname{AdS}}_{n}=\widetilde{\Lambda}$. On the other hand, since $x$ is in the invisible domain of $\widetilde{\Lambda}$, the set $J^{-}(x)$ is disjoint from $\widetilde{\Lambda}$. Therefore $J^{-}(x) \cap \mathrm{Cl}(\widetilde{E}(\widetilde{\Lambda}))$ is a compact subset of $\widetilde{\mathrm{AdS}}_{n}$. Therefore $J^{-}(x) \cap \mathrm{Cl}(\widetilde{E}(\widetilde{\Lambda}))$ is conformally equivalent to a compact causally convex domain in $\left(\mathbb{R} \times \mathbb{D}^{n-1},-d t^{2}+\right.$ $d s^{2}$ ) (with a bounded conformal factor since everything is compact). It immediately follows that the lengths of the past-directed causal curves starting at $x$ contained in $\widetilde{E}(\widetilde{\Lambda})$ is bounded (in other words, $\widetilde{\tau}(x)$ is finite), and that, for every past-oriented inextendible causal curve $c:[0,+\infty) \rightarrow \widetilde{E}(\widetilde{\Lambda})$ with $c(0)=x$, one has $\widetilde{\tau}(c(t)) \rightarrow 0$ when $t \rightarrow \infty$. This proves that $\widetilde{E}(\widetilde{\Lambda})$ has regular cosmological time.

Since the definition of AdS regular domains is "time-symmetric", $E(\Lambda)$ also has regular reverse cosmological time.

11.2. Horizons. According to Proposition 11.1 and Theorem 2.3, $E(\Lambda)$ is globally hyperbolic. Hence its boundary in $\mathrm{AdS}_{n}$ is a Cauchy horizon and enjoys all the known properties of Cauchy horizons (see for example [17]). In our framework, this boundary is the union of two closed achronal subsets, the past horizon $\mathcal{H}^{-}(\Lambda)$ and the future horizon $\mathcal{H}^{+}(\Lambda)$. Observe that $\mathcal{H}^{+}(\Lambda)$ is in the future of $\mathcal{H}^{-}(\Lambda)$.

In the conformal model $\left(\mathbb{D}^{2} \times \mathbb{S}^{1},-d t^{2}+d s^{2}\right)$, the horizons $\mathcal{H}^{-}(\Lambda)$ and $\mathcal{H}^{+}(\Lambda)$ are the graphs of the functions $f^{+}$and $f^{-}$defined in remark 10.5. In the Klein model, $E(\Lambda)$ is a convex domain, and the union $\mathcal{H}^{-}(\Lambda) \cup \mathcal{H}^{+}(\Lambda)$ is the topological boundary of this convex domain. We can therefore consider support hyperplanes to $E(\Lambda)$ at some point $p \in \mathcal{H}^{ \pm}(\Lambda)$. These are projective hyperplanes in $\mathbb{S}\left(\mathbb{R}^{n+1}\right)$. It is quite clear that, for such a support hyperplane $H \subset \mathbb{S}\left(\mathbb{R}^{n+1}\right)$, the corresponding totally geodesic hypersurface $H \cap \mathbb{A D S}_{n}$ is degenerate or spacelike (otherwise, $H$ would intersect transversally the achronal hypersurface $\mathcal{H}^{ \pm}(\Lambda)$, and this would contradict the fact that $H$ is a support hyperplane of $E(\Lambda))$.

The following is the analog of Lemma 7.1.

Proposition 11.2. Let $p$ a point of the past horizon $\mathcal{H}^{-}(\Lambda)$ of $E(\Lambda)$. Let $C(p) \subset T_{p} A d S$ be the set of the future directed unit tangent vectors orthogonal to the support hyperplanes of $E(\Lambda)$ at $p$. Then:

1. $C(p)$ is the convex hull of its lightlike elements.

2. If $c$ is a future complete geodesic ray starting at $p$ whose tangent vector at $p$ is a lightlike element of $C(p)$, then the future endpoint of $c$ is in $\Lambda$. 
Proof. First of all, we need to understand better the link between the way the elements of $C(p)$ are associated to the support planes of $E(\Lambda)$ at $p$. Let $H$ be a support hyperplane of $E(\Lambda)$ at $p$. Then $H=\mathbb{S}\left(u^{\perp}\right)$ where $u$ is an element of $\mathbb{R}^{n+1}$ such that

(i) $\langle u \mid u\rangle \leq 0$ (since $H=\mathbb{S}\left(u^{\perp}\right)$ is spacelike or lightlike);

(ii) $\langle p \mid u\rangle=0$ (since $p \in \mathbb{S}\left(u^{\perp}\right)$ );

(iii) $\langle x \mid u\rangle \leq 0$ for every $x \in E(\Lambda)$ (since $H=\mathbb{S}\left(u^{\perp}\right.$ ) is a support hyperplane of $E(\Lambda)$, and since, up to replacing $u$ by $-u$, we can assume that $u$ and $E(\Lambda)$ are on the same side of $H$ ).

Observe that this property (i) implies that the projection $[u]$ of $u$ in $\mathbb{S}\left(\mathbb{R}^{n}\right)$ belongs to $\mathbb{A D S}_{n} \cup \partial \mathbb{A D S}_{n}$. Also observe that $[u]$ and $E(\Lambda)$ being on the same side of $H$, the point $[u]$ must be in the future of $p$. Consider the 2-plane $P_{u}$ containing $u$ and $p$. The projection of $\mathbb{S}\left(P_{u}\right)$ of $P_{u}$ is a causal geodesic $\gamma_{u}$ containing $p$ and orthogonal to $H$. If $[u] \in \mathbb{A D S}_{n}$, then $[u] \in \gamma_{u}$; if $[u] \in \partial \mathbb{A D S}_{n}$, then $[u]$ is the final extremity of $\gamma_{u}$. We will denote by $v_{u}$ be the future directed unit tangent vector of $\gamma_{u}$ at $p$.

The set $C(p)$ is the set of all the vectors $v_{u}$ when $H=\mathbb{S}\left(u^{\perp}\right)$ ranges other the set of all the support hyperplanes of $E(\Lambda)$ at $p$. It is important to note that $v_{u}$ is lightlike if and only if $u$ is lightlike, i.e. if and only if $H=\mathbb{S}\left(u^{\perp}\right)$ is a lightlike hyperplane.

Now we will prove item (1). For this purpose, let us consider a support plane $H=S\left(u^{\perp}\right)$ of $E(\Lambda)$ at $p$. We know that $\langle x \mid u\rangle \leq 0$ for every $x \in E(\Lambda)$. We also know that $E(\Lambda)$ is the dual of the convex hull of $\Lambda$ in $\mathbb{S}\left(\mathbb{R}^{n}\right)$ (Proposition 10.17). This implies that the projection $[u] \in \mathbb{S}\left(\mathbb{R}^{n}\right)$ of $u \in \mathbb{R}^{n}$ belongs to the convex hull in $\mathbb{S}\left(\mathbb{R}^{n}\right)$ of $\Lambda$. Hence, we can write $u$ as a convex combination $u=\sum a_{i} u_{i}$ where the $u_{i}$ 's are elements of $\mathbb{R}_{n}$ projecting onto elements of $\Lambda$ and the $a_{i}$ are positive numbers (equivalently, $v_{u}$ is a convex combination of the $v_{u_{i}}$ 's). We know that the scalar $\langle p \mid u\rangle$ is equal to zero: $\sum a_{i}\left\langle p \mid u_{i}\right\rangle=0$. But all the terms of this sum are nonpositive. Therefore $\left\langle p \mid u_{i}\right\rangle=0$ for every $i$. As a consequence, $S\left(u_{i}^{\perp}\right)$ is a support plane of $E(\Lambda)$ at $p$ for every $i$ (equivalently, $v_{u_{i}}$ is an element of $C(p)$ for every $i$ ). Moreover, $H_{i}=\mathbb{S}\left(u_{i}^{\perp}\right)$ is a lightlike hyperplane for every $i$ (equivalently, $v_{u_{i}}$ is lightlike for every $i$ ). So, we have proved that $v_{u}$ is a convex combination of lightlike elements $C(p)$. This completes the proof of $(1)$.

It remains to prove item (2). For this purpose, we consider a support plane $H=S\left(u^{\perp}\right)$ of $E(\Lambda)$ at $p$, and the associated element $v_{u}$ of $C(p)$. We assume that $H$ is lightlike (equivalently that $v_{u}$ is lightlike). Just as above, we write $u=\sum a_{i} u_{i}$ where the $u_{i}$ 's projecting on elements of $\Lambda$, and the $a_{i}$ 's are positive. By hypothesis, the norm of $u$ is equal to zero: $\sum a_{i} a_{j}\left\langle u_{i} \mid u_{j}\right\rangle=0$. But, according to Proposition 10.16, the scalar product $\left\langle u_{i} \mid u_{j}\right\rangle$ is non-positive for every $i, j$. Hence, $\left\langle u_{i} \mid u_{j}\right\rangle$ must be equal to zero for every $i, j$. Hence, the subspace $F$ spanned by the $u_{i}$ 's is (totally) isotropic, which implies it is either 1-dimensional or 2-dimensional. In the first case, $\left[u_{i}\right]=\left[u_{j}\right]$ for all $i, j$, and in the second one, $S(F)$ is a lightlike geodesic containing all the $\left[u_{i}\right]$ 's. In both cases, we deduce that $[u]$ belongs to the segment joining $\left[u_{i}\right]$ to $\left[u_{j}\right]$ for some $i, j$. It follows that $[u]$ belongs to $\Lambda$ (since $\Lambda$ is achronal, every lightlike segment with both ends in $\Lambda$ is contained in $\Lambda$ ). Now recall that $v_{u}$ is the tangent vector at $p$ of the geodesic segment joining $p$ and $[u]$. Hence, we have proved that the future extremity of the lightlike ray starting at $p$ with tangent vector $v_{u}$ lies in $\Lambda$. This completes the proof of (2).

REMARK 11.3. Of course, a similar statement holds for the future horizon $\mathcal{H}^{+}(\Lambda)$ but where complete null rays contained in the horizon are now past oriented. 
11.3. Retraction onto the horizon. According to (3) in Theorem 2.3, for every point $x$ in the regular domain, there exists at least one maximal timelike geodesic ray with future endpoint $x$ realizing the "distance to the initial singularity": we call such a geodesic ray a realizing geodesic for $x$.

Definition 11.4. The region $\{\tau<\pi / 2\}$ of the AdS regular domain $E(\Lambda)$ is denoted $E_{0}^{-}(\Lambda)$ and called the past tight region of $E(\Lambda)$.

Proposition 11.5. Let $x$ be an element of the past tight region $E_{0}^{-}(\Lambda)$ of $E(\Lambda)$. Then, there is an unique realizing geodesic for $x$.

This proposition means that the past tight region is foliated by inextendible timelike geodesics on which $\tau$ restricts as a unit speed parameter.

Proof. Consider an affine domain $U$ containing $E(\Lambda)$ (see Proposition 10.12). In some coordinate system $\left(u_{1}, u_{2}, \ldots, u_{n}\right)$ the domain $U$ is the region $\left\{-u_{1}^{2}+u_{2}^{2}+\ldots+\right.$ $u_{n}^{2}<1$ \}, and $x$ has zero coordinates (see definition 10.10 and remark 10.11). Initial extremities of realizing geodesics for $x$ are points $z$ in $\mathcal{H}^{-}(\Lambda)$ such that $d(x, z)=\tau(x)$, where $d(x, z)$ is the time length of a past oriented timelike geodesic in $\mathbb{A D S}_{n}$ starting from $x$ and ending to $z$ (hence $d(x, z)=0$ if $z$ is not in the past of $x$ ). For each $\tau$, we have: $\mathcal{E}_{\tau}=\{z \in U \mid d(x, z) \geq \tau\}=\left\{-u_{1}^{2}+u_{2}^{2}+\ldots+u_{n}^{2} \leq-\tan ^{2}(\tau), x_{1}<0\right\}$. If $\tau<\tau^{\prime}$, then $\mathcal{E}_{\tau^{\prime}} \subset \mathcal{E}_{\tau}$. Since $E(\Lambda)$ is causally convex, one has:

$$
\tau(x)=\sup \left\{\tau \mid \mathcal{E}_{\tau} \cap \mathcal{H}_{-}(\Lambda) \neq \emptyset\right\}
$$

Let $y, y^{\prime}$ be initial extremities of realizing geodesics for $x$ : they both belong to $\mathcal{E}_{\tau(x)} \cap$ $E(\Lambda)$. Assume by contradiction that $y \neq y^{\prime}$, and take any element $z$ in the interior of the segment $\left[y, y^{\prime}\right]$. On the one hand, since $E(\Lambda)$ is geodesically convex, $z$ belongs to $E(\Lambda)$. On the other hand, $z$ belongs to the interior of $\mathcal{E}_{\tau(x)}$ (since the hyperboloid $\left\{-u_{1}^{2}+u_{2}^{2}+\ldots+u_{n}^{2}=-\tan ^{2}(\tau(x)), x_{1}<0\right\}$ is concave). Hence, the length of the geodesic segment $[x, z]$ is strictly bigger than $\tau(x)$. Contradiction. $\mathbf{\square}$

Proposition 11.6. Let $c:(0, T] \rightarrow E_{0}^{-}(\Lambda)$ be a future oriented timelike geodesic whose initial extremity $p:=\lim _{t \rightarrow 0} c(t)$ is in the past horizon $\mathcal{H}^{-}(\Lambda)$. Then the following assertions are equivalent.

1. For every $t \in(0, T], c_{\mid[0, t]}$ is a realizing geodesic for the point $c(t)$.

2. There exists $t_{0} \in(0, T]$ such that $c_{[0, t]}$ is a realizing geodesic for the point $c(t)$.

3. $c$ is orthogonal to a support hyperplane of $E(\Lambda)$ at $p:=\lim _{t \rightarrow 0} c(t)$.

Proof. Obviously (1) $\Rightarrow(2)$.

Assume that there exists $t_{0} \in(0, T]$ such that $c_{\mid[0, t]}$ is a realizing geodesic for the point $c\left(t_{0}\right)$. Let $x:=c\left(t_{0}\right)$ and $p:=\lim _{t \rightarrow 0} c(t)$. The level set $\{z \mid d(x, z)=\tau(x)\}$ is a smooth hypersurface in $\mathbb{A D S}_{n}$ and its tangent space at $p$ is the orthogonal in $T_{p} \mathbb{A D S}_{n}$ of the vector tangent to $c$. If this tangent space is not tangent to a support hyperplane of $\mathcal{H}^{-}(\Lambda)$ then, the set $\{d(x, z)=\tau(x)\}$ intersects $E(\Lambda)$. This would imply that $p$ is not a minimum point for the restriction of $d(x,$.$) to E(\Lambda)$. This is a contradiction since the restriction of $c\left(\left(0, t_{0}\right]\right)$ is a realizing geodesic for $x$. Hence $(2) \Rightarrow(3)$.

Now assume that $c$ is orthogonal to a support hyperplane of $\mathcal{H}^{-}(\Lambda)$ at $p$, and let $x$ be a point of $c$. Consider an affine domain $U$ centered at $x$. The hyperboloid $\{z \in U \mid d(x, z)=d(x, p)\}$ is orthogonal to $c$ at $p$ : hence, by hypothesis, its tangent space at $p$ is a support hyperplane of $E(\Lambda)$. Since $\mathcal{H}^{-}(\Lambda)$ is convex whereas the hyperboloid is strictly concave, the intersection of $\mathcal{E}(\Lambda)$ with $\mathcal{H}^{-}(\Lambda)$ is $\{p\}$. This 
means that $p$ is a minimum point for $d(x,$.$) . Therefore, [x, y]$ is a realizing geodesic for $x$. Hence $(3) \Rightarrow(1)$.

REMARK 11.7. Using the reverse cosmological time $\widehat{\tau}$ instead of $\tau$, one can define the future tight region $E_{0}^{+}(\Lambda)$ of $E(\Lambda)$, and prove some analogs of Propositions 11.5 and 11.6.

12. AdS regular domains: curvature estimates for cosmological time level sets. We are now able to state the main result on curvature estimates of the level sets of the cosmological time of an AdS regular domain.

THEOREM 12.1. Let $E^{-}(\Lambda)$ be the past tight region of an AdS regular domain, and $\tau: E_{0}^{-}(\Lambda) \rightarrow(0, \pi / 2)$ be the associated cosmological time. For every $a \in(0, \pi / 2)$, the generalized mean curvature of the level set $S_{a}=\tau^{-1}(a)$ satisfies

$$
-\cot (a) \leq H_{S_{a}} \leq-\frac{1}{n-1} \cot (a)+\frac{n-2}{n-1} \tan (a) .
$$

Proof. Let $x$ be a point on the level set $S_{a}$. We denote by $c:[0, a] \rightarrow E^{-}(\Lambda)$ the unique realizing geodesic for $x$, with initial extremity $p=r(x)$. Let $v$ be the future oriented unit speed tangent vector of $c$ at $p$. We denote as before $C(p)$ the set of vectors in $T_{p} \mathbb{A D S}_{n}$ orthogonal to support hyperplanes of the past horizon at $p$. Our goal is to construct two local surfaces $\mathbb{S}_{x}^{+}, \mathbb{S}_{x}^{-}$containing $x$, respectively in the future and the past of $S_{a}$, and with known mean curvature at $x$ (recall Definition 3.1).

Construction of $\mathbb{S}_{x}^{+}$. The construction of the upper barrier $\mathbb{S}_{x}^{+}$is similar to the construction in the flat case: take a portion near $x$ of the set of points at lorentzian distance $a$ from $p=r(x)$. The mean curvature of $\mathbb{S}_{x}^{+}$is $-\cot (a)$, its tangent hyperplane at $x$ is the hyperplane orthogonal to $c$ at $x$.

Construction of $\mathbb{S}_{x}^{-}$. Let $\widetilde{\Lambda}$ be a lift of $\Lambda$ in $\partial \widetilde{\mathrm{AdS}}{ }_{n} \simeq \mathbb{R} \times \mathbb{S}^{n-2}$. We recall that $\widetilde{\Lambda}$ can be seen as the graph of a 1-Lipschitz function $f: \mathbb{S}^{n-2} \rightarrow \mathbb{R}$. By Proposition 11.6, the vector $v$ is in $C(p)$. Hence, Proposition 11.2 implies that there is a finite set $\left\{v_{1}, \ldots, v_{l}\right\}$ of lightlike elements of $C(p)$ such that $v$ is in the convex hull of $\left\{v_{1}, \ldots, v_{l}\right\}$. According to Proposition 11.2 the future extremities of the lightlike geodesics whose tangent vectors at $p$ are $v_{1}, \ldots, v_{l}$ belong to $\Lambda$. Let $B$ be the finite subset of $\Lambda$ made of these future extremities, and $\widetilde{B}$ the corresponding subset of $\widetilde{\Lambda}$. Then $\widetilde{B}$ is the graph of a 1-Lipschitz function $f_{B}: B_{0} \rightarrow \mathbb{R}$ where $B_{0}$ is a finite subset of $\mathbb{S}^{n-2}$ (see remark 10.2). Let $\widetilde{\Lambda}_{B}$ be the graph of the $f_{B}^{-}: \mathbb{S}^{n-2} \rightarrow \mathbb{R}$ defined remark 10.5 , and $\Lambda_{B}$ be the projection of $\widetilde{\Lambda}_{B}$. We define our hypersurface $\mathbb{S}_{x}^{-}$to be the $a$-level set of the cosmological time of the domain $E\left(\Lambda_{B}\right)$.

Let us check that $\mathbb{S}_{x}^{-}$satisfies the required properties: $x \in \mathbb{S}_{x}^{-}$and $\mathbb{S}_{x}^{-}$is in the past of $S_{a}$. Since $\Lambda_{B}$ subset $\Lambda$, the invisible domain $E\left(\Lambda_{B}\right)$ contains the invisible domain $E(\Lambda)$, and hence the hypersurface $\mathbb{S}_{x}^{-}$is in the past of the hypersurface $S_{a}$. For each $x \in \Lambda_{B}$, there is a future directed lightlike geodesic ray starting at $p$ whose endpoint is equal to $x$. It follows that $p \in \mathcal{H}^{-}\left(\Lambda_{B}\right)$. By construction, the vectors $v_{1}, \ldots, v_{l}$ are orthogonal to support hyperplanes of $E\left(\Lambda_{B}\right)$ at $p$. Hence $v \in \operatorname{Conv}\left(v_{1}, \ldots, v_{l}\right)$ is also orthogonal to a support hyperplane of $E\left(\Lambda_{B}\right)$ at $p$. According to Proposition 11.6, this implies that $c$ is a realizing geodesic in $E\left(\Lambda_{B}\right)$. It follows that $x=c(a)$ belongs to the $a$-level set of the cosmological time of $E\left(\Lambda_{B}\right)$, i.e. $x \in \mathbb{S}_{x}^{-}$.

We are left to evaluate the mean curvature of the hypersurface $\mathbb{S}_{x}^{-}$at $x$. The finite set $B$ is the projection of a set $\widehat{B}$ of null vectors in $E_{n}$. Let $F$ be the vector 
space spanned by $\widehat{B}$, and let $F^{\perp}$ be the subspace orthogonal to $F$. Let $1+d$ be the dimension of $F$. The convex hull of $\widehat{B}$ contains a timelike element $\hat{q}$ with $Q_{2, n-1}$-norm -1 : the dual to the spacelike support hyperplane at $p$ orthogonal to $v$. This point $\hat{q}$ can also be defined as the unique element of $\mathrm{AdS}_{n}$ projecting on $q=c(\pi / 2)$.

Similarly, $F^{\perp}$ contains a timelike vector: the lift $\hat{p}$ in $E_{n}$ of $p$, let us say, $Q_{2, n-1}(\hat{p})=-1$. It follows that $F \cap F^{\perp}=\{0\}, F$ has signature $(1, d)$, and $F^{\perp}$ has signature $(1, n-d-1)$.

Let $G \approx \mathrm{SO}_{0}(1, n-d-1)$ be the subgroup of $\mathrm{SO}_{0}(2, n-1)$ made of the elements acting trivially on $F$. The group $G$ preserves $\widehat{B}$. It follows that its induced action on $S(E)$ preserves $E\left(\Lambda_{B}\right)$. This action preserves the cosmological time $\tau_{B}$ of $E\left(\Lambda_{B}\right)$. The $G$-orbit of $p$ is a connected component of the geodesic subspace $S\left(F^{\perp}\right) \cap \mathbb{A D S}_{n}$.

Let $F_{1}$ be the subspace $F^{\perp} \oplus\langle\hat{q}\rangle$. Observe that $\hat{q}$ is a fixed point for the action of $G$. The projection $A_{1}$ of $F_{1} \cap \mathrm{AdS}_{n}$ in $S\left(E_{n}\right)$ is a copy of the Klein model of the anti de Sitter space of dimension $n-d$. It contains $x$ which is the projection of $\hat{x}=\cos (a) \hat{p}+\sin (a) \hat{q}$. The $G$-orbit of $x$ is contained in the cosmological level $\tau_{B}^{-1}(a)$. On the other hand, this $G$-orbit in the anti de Sitter space $A_{1}$ is the set of initial extremities of future oriented timelike geodesics with future extremity $q$ and of length $\pi / 2-a$. Hence, it is an umbilical submanifold with principal curvatures $\cot (\pi / 2-a)=\tan (a)$. This $G$-orbit is orthogonal to $r^{-1}(p)$, and in $r^{-1}(p) \subset S(F)$, the cosmological time $\tau_{B}$ is simply the lorentzian distance to $p: \tau_{B}^{-1}(a) \cap r^{-1}(p)$ is an umbilical submanifold with principal curvatures $-\cot (a)$. Hence, the mean curvature of $\mathbb{S}_{x}^{-}=\tau_{B}^{-1}(a)$ at points in $r^{-1}(p)$ is

$$
\left.-\frac{d}{n-1} \cot (a)+\frac{n-d-1}{n-1} \tan (a)\right)
$$

and the same is true at all points of $\tau_{B}^{-1}(a)$ because of the $G$-invariance. In order to conclude, we just need to observe that

$$
\left.\left.-\frac{d}{n-1} \cot (a)+\frac{n-d-1}{n-1} \tan (a)\right) \leq-\frac{1}{n-1} \cot (a)+\frac{n-1}{n-2} \tan (a)\right)
$$

since $a \in(0, \pi / 2)$ and $d \in\{1, \ldots, n-1\}$.

Reversing the time in the proof of Theorem 12.1, one gets:

THEOREM 12.2. Let $E^{+}(\Lambda)$ be the future tight region of an AdS regular domain, and $\widehat{\tau}: E^{+}(\Lambda) \rightarrow(0, \pi / 2)$ be the associated reverse cosmological time. For every $a \in(0, \pi / 2)$, the generalized mean curvature of the level set $\widehat{S}_{a}=\widehat{\tau}^{-1}(a)$ satisfies

$$
\frac{1}{n-1} \cot (a)-\frac{n-2}{n-1} \tan (a) \leq H_{\widehat{S}_{a}} \leq \cot (a)
$$

13. CMC time functions in anti-de Sitter MGHC spacetimes. The proof follows the same lines as those of Theorem 1.4, but is slightly complicated by the fact that we need to consider also the reverse cosmological time (cf. remark 2.4).

Proof of Theorem 1.7. Let $(M, g)$ be a $n$-dimensional MGHC spacetimes with constant curvature -1 . According to Theorem $10.18,(M, g)$ is the quotient of a regular domain $E(\Lambda) \subset \operatorname{AdS}_{n}$ by a torsion-free discrete group $\Gamma \subset \operatorname{Isom}\left(\operatorname{AdS}_{n}\right)$. The cosmological time $\tau: E(\Lambda) \rightarrow(0,+\infty)$ and the reverse cosmological time $\widehat{\tau}: E(\Lambda) \rightarrow$ $(0,+\infty)$ are well-defined and regular (Proposition 11.1). 
For every $a \in[0,+\infty]$, let $S_{a}=\tau^{-1}(a)$ and $\Sigma_{a}$ be the projection of $S_{a}$ in $M \equiv$ $\Gamma \backslash E(\Lambda)$. Every level set $S_{a}$ is quite obviously a Cauchy hypersurface in $E(\Lambda)$. It is $\Gamma$-invariant since the cosmological time is so. It follows that $\Sigma_{a}$ is a topological Cauchy hypersurface in $M$ since inextendible causal curves in $M$ are projections of inextendible causal curves in $E(\Lambda)$. Moreover, Theorem 12.1 implies that the generalized mean curvature $H_{\Sigma_{a}}$ of $\Sigma_{a}$ satisfies

$$
-\cot (a) \leq H_{\Sigma_{a}} \leq-\frac{\cot (a)}{n-1}+\frac{n-2}{n-1} \tan (a)
$$

Consider a decreasing sequence of positive real numbers $\left(a_{m}\right)_{m \in \mathbb{N}}$ such that $a_{m} \rightarrow 0$ when $m \rightarrow+\infty$. Observe that

$$
-\frac{\cot \left(a_{m}\right)}{n-1}+\frac{n-2}{n-1} \tan \left(a_{m}\right) \underset{m \rightarrow \infty}{\longrightarrow}-\infty
$$

This shows that $\left(\Sigma_{a_{m}}\right)_{m \in \mathbb{N}}$ is a sequence of past asymptotic $(-\infty)$-barriers.

For every $a \in[0,+\infty]$, let $\widehat{S}_{a}=\widehat{\tau}^{-1}(a)$ and $\widehat{\Sigma}_{a}$ be the projection of $\widehat{S}_{a}$ in $M$. Of course, $\widehat{\Sigma}_{a}$ is a topological Cauchy hypersurface in $M$ for every $a$. By Theorem 12.2, the generalized mean curvature $H_{\widehat{\Sigma}_{a}}$ of $\widehat{\Sigma}_{a}$ satisfies

$$
\frac{1}{n-1} \cot (a)-\frac{n-2}{n-1} \tan (a) \leq H_{\Sigma_{a}} \leq \cot (a)
$$

Consider a decreasing sequence of positive real numbers $\left(b_{m}\right)_{m \in \mathbb{N}}$ such that $b_{m} \rightarrow 0$ when $m \rightarrow+\infty$. Observe that

$$
\frac{1}{n-1} \cot \left(b_{m}\right)-\frac{n-2}{n-1} \tan \left(b_{m}\right) \underset{m \rightarrow \infty}{\longrightarrow}+\infty .
$$

This shows that $\left(\widehat{\Sigma}_{b_{m}}\right)_{m \in \mathbb{N}}$ is a sequence of past asymptotic $(+\infty)$-barriers.

So we are in a position to apply Theorem 4.3, which shows that $M$ admits a globally defined CMC-time $\tau_{c m c}: M \rightarrow(-\infty,+\infty)$.

14. Description of de Sitter MGHC spacetimes. We now start our investigation of MGHC de Sitter spacetimes (i.e. MGHC spacetimes with constant curvature $+1)$. Each section in the sequel is a "de Sitter substitute" of a section above dealing with anti-de Sitter spacetimes. Our first task will be to introduce a de Sitter analog of the notion of $A d S$ regular domain, called $d S$ standard spacetime. Every MGHC de Sitter spacetime is the quotient of a dS standard spacetime by a torsion free subgroup of $\operatorname{Isom}_{0}\left(\mathrm{dS}_{n}\right)=\mathrm{O}_{0}(1, n)$. Then, we will try to get a good understanding of the geometry of dS standard spacetimes, in order to obtain some estimates of the (generalized) mean curvature of the level sets of the cosmological time.

In comparison to the anti-de Sitter case, a major technical difficulty appears: given a MGHC de Sitter spacetime $(M, g)$, the developing map $D: \widetilde{M} \rightarrow \mathrm{dS}_{n}$ is not one-to-one in general. A consequence is that dS standard spacetimes cannot be defined as domains in the de Sitter space $\mathrm{dS}_{n}$. A dS standard spacetime is a simply connected manifold which is locally isometric to $\mathrm{dS}_{n}$; in some particular cases, this manifold is globally isometric to an open domain in $\mathrm{dS}_{n}$, but this is not the general case. 
14.1. dS standard spacetimes. The purpose of this section is to define a class of locally de Sitter manifolds, called $d S$ standard spacetimes. Recall that a Möbius manifold is a manifold equipped with a $(G, X)$-structure, where $X=\mathbb{S}^{n-1}$ is the $(n-1)$-dimensional sphere and $G \equiv \mathrm{O}_{0}(1, n)$ is the Möbius group (i.e. the group of transformations preserving the usual conformal structure of $\left.\mathbb{S}^{n-1}\right)$. To every $(n-1)$ dimensional simply connected Möbius manifold $S$, we will associate a $n$-dimensional future complete dS standard spacetimes $\mathcal{B}_{0}^{+}(S)$ diffeomorphic to $S \times \mathbb{R}$. A similar construction leads to a $n$-dimensional past complete dS standard spacetime $\mathcal{B}_{0}^{-}(S)$.

The definition of dS standard spacetimes we will use here first appeared in a paper by Kulkarni and Pinkall (see $\S 3.4$ of [38]). Unfortunately, Kulkarni-Pinkall did not insist on the de Sitter nature of the space they consider, and we need to formulate here the lorentzian interpretation of some of their results. There is another construction by Scannell (generalizing some ideas of Mess; see [45] and [40]) where the de Sitter nature of the resulting spaces is obvious. But Scannell only considered the case where $S$ is closed, and it is not obvious from his description that the obtained spacetimes are past or future complete. So, we will reproduce here Kulkarni-Pinkall's and Scannell's constructions, for the readers' convenience, and in order to ensure that both these constructions lead to the same spacetimes.

14.2. Linear and Klein models of the de Sitter space. For $n \geq 2$, let $\left(x_{1}, \ldots, x_{n+1}\right)$ be the standard coordinate system on $\mathbb{R}^{n+1}$, and let $Q_{1, n}$ be the quadratic form $-x_{1}^{2}+x_{1}^{2}+\ldots+x_{n+1}^{2}$. The linear model of the $n$-dimensional de Sitter space is the one-sheeted hyperboloid $\left(Q_{1, n}=+1\right)$ endowed with the lorentzian metric induced by $Q_{1, n}$; we denote it by $\mathrm{dS}_{n}$.

It is easy to check that $\mathrm{dS}_{n}$ is homeomorphic to $\mathbb{R} \times \mathbb{S}^{n-1}$. Actually, one can prove that $d S_{n}$ is conformally equivalent to $\left((-\pi / 2, \pi / 2) \times \mathbb{S}^{n-1},-d t^{2}+d s^{2}\right)$, where $d t^{2}$ is the usual metric on $\mathbb{R}$ and $d s^{2}$ is the usual metric (of curvature 1 ) on the sphere $\mathbb{S}^{n-1}$. It follows in particular that $\mathrm{dS}_{n}$ is globally hyperbolic. The coordinate $x_{0}$ defines on $\mathrm{dS}_{n}$ a time function (provided that we make the appropriate choice of time-orientation).

Observe that each of the two sheets of the hyperboloid $\left(Q_{1, n}=-1\right)$ endowed with the riemannian metric induced by $Q_{1, n}$ is a copy of the $n$-dimensional hyperbolic space. We denote by $H_{n}^{-}$(resp. $\left.H_{n}^{+}\right)$the sheet of the hyperboloid $\left(Q_{1, n}=-1\right)$ contained in the half space $\left(x_{0}<0\right)$ (resp. $\left.x_{0}>0\right)$.

The projection on $\mathbb{S}\left(\mathbb{R}^{n+1}\right)$ of $\mathrm{dS}_{n}$ (endowed with the push-forward of the lorentzian metric of $\mathrm{dS}_{n}$ ) is the Klein model of the de Sitter space; we denote it by $\mathbb{D S}_{n}$. The projections on $\mathbb{S}\left(\mathbb{R}^{n+1}\right)$ of $H_{-}^{n}$ and $H_{+}^{n}$ will be denoted by $\mathbb{H}_{-}^{n}$ and $\mathbb{H}_{+}^{n}$. The boundary of $\mathbb{D S}_{n}$ in $\mathbb{S}\left(\mathbb{R}^{n-1}\right)$ is the projection of the cone $\left(Q_{1, n}=0\right) \backslash\{0\}$; this is the union of two spheres $\mathbb{S}_{+}^{n-1}, \mathbb{S}_{-}^{n-1}$. We choose the notations such that $\mathbb{S}_{+}^{n-1}$ (resp. $\mathbb{S}_{-}^{n-1}$ ) is included in the projection of the half space $x_{0}>0$ (resp. $\left.x_{0}<0\right)$. Notice that $\mathbb{S}_{+}^{n-1}$ (resp. $\mathbb{S}_{-}^{n-1}$ ) is also the boundary of $\mathbb{H}_{-}^{n}$ (resp. $\left.\mathbb{H}_{-}^{n}\right)$ in $\mathbb{S}\left(\mathbb{R}^{n+1}\right)$.

Using the conformal structure of $\mathrm{dS}_{n}$, one sees that every future oriented inextendible causal curve in $\mathbb{D S}_{n}$ "goes from $\mathbb{S}_{-}^{n-1}$ to $\mathbb{S}_{+}^{n-1}$ ". In other words, $\mathbb{S}_{+}^{n-1}$ can be seen as the future boundary of $\mathbb{D S}_{n}$, and $\mathbb{S}_{-}^{n-1}$ as the past boundary.

An important observation is that the group $\mathrm{O}_{0}(1, n)$ can be seen alternatively as the isometry group of the lorentzian space $\mathbb{D S}_{n}$, as the isometry group of the hyperbolic spaces $\mathbb{H}_{-}^{n}$ and $\mathbb{H}_{+}^{n}$, or as the Möbius group of the spheres $\mathbb{S}_{-}^{n-1}$ and $\mathbb{S}_{+}^{n-1}$ (i.e. the group of the transformations preserving the usual conformal structure on the spheres $\mathbb{S}_{-}^{n-1}$ and $\mathbb{S}_{+}^{n-1}$ ). In other words, each isometry of $\mathbb{D S}_{n}$ extends as a 
conformal tranfomation of the spheres $\mathbb{S}_{-}^{n-1}$ and $\mathbb{S}_{+}^{n-1}$, and conversely, each conformal tranformation of the sphere $\mathbb{S}_{ \pm}^{n-1}$ extends as an isometry of $\mathbb{D S}_{n}$.

The geodesics of $\mathbb{D S}_{n}$ are the connected components of the intersections of $\mathbb{D S}_{n}$ with the projective lines of $\mathbb{S}\left(\mathbb{R}^{n+1}\right)$. More precisely, let $\gamma$ be a projective line in $\mathbb{S}\left(\mathbb{R}^{n+1}\right)$, then

- if $\gamma$ does not intersect the spheres $\mathbb{S}_{-}^{n-1}$ and $\mathbb{S}_{+}^{n-1}$, then $\gamma$ is a spacelike geodesic of $\mathbb{D S}_{n}$,

- if $\gamma$ is tangent to the spheres $\mathbb{S}_{-}^{n-1}$ and $\mathbb{S}_{+}^{n-1}$, then each of the two connected components of $\gamma \cap \mathbb{D S}_{n}$ is a lightlike geodesic in $\mathbb{D S}_{n}$,

- if $\gamma$ intersects transversally the spheres $\mathbb{S}_{-}^{n-1}$ and $\mathbb{S}_{+}^{n-1}$, then each of the two connected components of $\gamma \cap \mathbb{D S}_{n}$ is a timelike geodesic.

The causal future $J^{+}(x)$ of a point $x \in \mathbb{D S}_{n}$ is the union of all the projective segments contained in $\mathbb{D S}_{n}$, joining $x$ to $\mathbb{S}_{+}^{n-1}$. For the timelike future $I^{+}(x)$, one only considers the segments that hit the $\mathbb{S}_{+}^{n-1}$ transversally. The totally geodesic hypersurfaces in $\mathbb{D S}_{n}$ are the connected components of the intersections of $\mathbb{D S}_{n}$ with the projective hyperplanes of $\mathbb{S}\left(\mathbb{R}^{n+1}\right)$.

A key ingredient in the sequel will be the fact that de Sitter space can be thought of as the space of (non-trivial open) round balls in $\mathbb{S}_{+}^{n-1}$. For every point $x \in \mathbb{D S}_{n}$, we denote by $\partial^{+} I^{+}(x)$ the set of the future endpoints in $\mathbb{S}_{+}^{n-1}$ of all the future oriented timelike geodesic rays starting at $x$. Then, for every $x \in \mathbb{D S}_{n}$, the set $\partial^{+} I^{+}(x)$ is an open round ball in $\mathbb{S}_{+}^{n-1}$. One can easily check that the map associating to $x$ the round ball $\partial^{+} I^{+}(x)$ establishes a one-to-one correspondance between the points in $\mathbb{D S}_{n}$ and the (non-trivial open) round balls in $\mathbb{S}_{+}^{n-1}$. Observe that a point $x \in \mathbb{D S}_{n}$ is in the (causal) past of another point $y \in \mathbb{D S}_{n}$, if and only if the round ball associated to $x$ contains the round ball associated to $y$. Of course, there is a similar identification between the points of $\mathbb{D S}_{n}$ and the round balls in $\mathbb{S}_{-}^{n-1}$.

14.3. dS standard spacetimes associated to open domains in $\mathbb{S}_{+}^{n-1}$. Recall that our goal is to associate a future complete dS standard spacetime $\mathcal{B}_{0}^{+}(S)$ to every simply connected Möbius manifold $S$. In this paragraph, we consider the particular case where $S$ is an open domain in the sphere $\mathbb{S}_{+}^{n-1}$. We denote by $\Lambda$ the boundary of $S$ in $\mathbb{S}_{+}^{n-1}$.

For $p \in \Lambda$, let $H(p)$ be the unique projective hyperplane in $\mathbb{S}\left(\mathbb{R}^{1, n}\right)$ tangent to $\mathbb{S}_{+}^{n-1}$ at $p$. Note that $H(p) \cap \mathbb{S}_{+}^{n-1}=\{p\}, H(p) \cap \mathbb{S}_{-}^{n-1}=\{-p\}$, and $H(p) \backslash\{p,-p\}$ is contained in $\mathbb{D S}_{n}$ (more precisely, $H(p) \backslash\{p,-p\}$ is a lightlike totally geodesic hypersurface in $\left.\mathbb{D S}_{n}\right)$. Also note that $\mathbb{S}\left(\mathbb{R}^{n+1}\right) \backslash H(p)$ has two connected components. We denote by $\Omega^{+}(p)$ the connected component of $\mathbb{S}\left(\mathbb{R}^{n+1}\right) \backslash H(p)$ containing $\mathbb{H}_{+}^{n}$.

DeFinition 14.1. We consider the set

$$
\Omega^{+}(S):=\bigcap_{p \in \Lambda} \Omega^{+}(p)
$$

We denote by $\mathcal{B}_{0}^{+}(S)$ the unique connected component of $\Omega^{+}(S) \cap \mathbb{D S}_{n}$ whose closure in $\mathbb{S}\left(\mathbb{R}^{1, n}\right)$ contains $S$ (see remark 14.2 below). The domain $\mathcal{B}_{0}^{+}(S)$ is the (future complete) $d S$ standard spacetime associated to $S$.

REMARK 14.2. The set $\Omega^{+}(S)$ is obviously a convex domain of $S\left(\mathbb{R}^{1, n}\right)$. This convex domain contains the hyperbolic space $\mathbb{H}_{+}^{n}$. Select a point $O \in \mathbb{H}_{+}^{n}$. The radial projection of center $O$ on $\mathbb{S}_{+}^{n-1}$ defines a fibration of $\Omega^{+}(S) \cap \mathbb{D S}_{n}$ over $\mathbb{S}_{+}^{n-1} \backslash \Lambda$ with 
fibers $\mathbb{R}$. It follows that there exists a unique connected component of $\Omega^{+}(S) \cap \mathbb{D} \mathbb{S}_{n}$ whose closure contains $S$. This shows the validity of the above definition of $\mathcal{B}_{0}^{+}(S)$.

REMARK 14.3. Since geodesic segments in $\mathbb{D S}_{n}$ are segments of projective lines, another consequence of the convexity of $\Omega^{+}(S)$ is the geodesic convexity of $\mathcal{B}_{0}^{+}(S)$ : any geodesic segment joining two elements of $\mathcal{B}_{0}^{+}(S)$ is contained in $\mathcal{B}_{0}^{+}(S)$.

REMARK 14.4. For every $p \in \Lambda$, the set $\Omega^{+}(p) \cap \mathbb{D S}_{n}$ is the timelike future of the hyperplane $H(p)$ in $\mathbb{D S}_{n}$. It follows that, for every $x \in \mathcal{B}_{0}^{+}(S)$, the causal future of $x$ in $\mathbb{D S}_{n}$ is contained in $\mathcal{B}_{0}^{+}(S)$. Since $\mathbb{D S}_{n}$ is future complete, it also follows that $\mathcal{B}_{0}^{+}(S)$ is future complete.

REMARK 14.5. For every $p \in \Lambda$, one has

$$
\Omega^{+}(p)=\left\{x \in \mathbb{S}\left(\mathbb{R}^{n+1}\right) \text { such that }\langle x \mid p\rangle<0\right\}
$$

where $\langle\cdot \mid \cdot\rangle$ is the pseudo-scalar product associated to the quadratic form $Q_{1, n}$. It follows that $\Omega^{+}(S)$ is the dual convex set of the convex hull of $\Lambda$ in $\mathbb{S}\left(\mathbb{R}^{n+1}\right)$.

REMARK 14.6. One can easily check that $\Omega^{+}(S) \cap \mathbb{D S}_{n}$ is the set of points in $\mathbb{D S}_{n}$ which are not causally related to any element of $\Lambda$. Therefore, $\mathcal{B}_{0}^{+}(S)$ can be considered as the domain of dependence of $S$ in $\mathbb{D S}_{n}$, so that there is a complete analogy between the above definition of $\mathrm{dS}$ standard spacetimes and the definition of AdS regular domains.

REMARK 14.7. Recall that there is a canonical identification between the points of $\mathbb{D S}_{n}$ and the round balls in $\mathbb{S}_{+}^{n-1}$ (see section 14.2 ). One can easily check that a point $x \in \mathbb{D S}_{n}$ is in $\mathcal{B}_{0}^{+}(S)$ if and only if the ball of $\mathbb{S}_{+}^{n-1}$ corresponding to $x$ is contained in $S$.

Of course, there is a similar construction which allows to associate a past complete domain $\mathcal{B}_{0}^{-}(S)$ to any connected open domain $S$ in $\mathbb{S}_{-}^{n-1}$.

14.4. The general case. Now we consider the general case where $S$ is any simply connected $(n-1)$-dimensional Möbius manifold. A key ingredient will be the identification between $\mathbb{D S}_{n}$ and the set of round balls in $\mathbb{S}_{+}^{n-1}$.

Let us first state two technical lemmas, valid for any local homeomorphism $\varphi$ : $X \rightarrow Y$ between manifolds (for proofs, see e.g. [10, $\S 2.1]$ ).

LEMMA 14.8. Let $U, U^{\prime}$ be two open domains in $X$, such that $\varphi$ is one-to-one in restriction to $U$, and in restriction to $U^{\prime}$. Assume that $U \cap U^{\prime}$ is not empty, and that $\varphi\left(U^{\prime}\right)$ contains $\varphi(U)$. Then, $U^{\prime}$ contains $U$.

LEMMA 14.9. Assume that $\varphi$ is one-to-one in restriction to some open domain $U$ in $X$. Also assume that the set $V=\varphi(U)$ is locally connected in $Y$, i.e. every point $y$ in the closure of $V$ admits arbitrarly small neighborhood $W$ such that $V \cap W$ is connected. Then, the restriction of $\varphi$ to the closure of $U$ in $X$ is one-to-one.

Now we start the construction of the dS standard spacetime $\mathcal{B}_{0}^{+}(S)$. For this purpose, we choose a developing map $d: S \rightarrow \mathbb{S}_{+}^{n-1}$. Recall that such a map does exist since $S$ is a Möbius manifold. Also recall that the map $d$ is not one-to-one in general. 
Definition 14.10. An (open) round ball $U$ in $S$ is an open domain in $S$ such that the developing map $d$ to $U$ is one-to-one in restriction to $U$, and such that $d(U)$ is an open round ball in $\mathbb{S}_{+}^{n-1}$. A round ball $U \subset S$ is said to be proper if the image under $d$ of the closure $\bar{U}$ of $U$ in $S$ is the closure of $d(U)$ in $\mathbb{S}_{+}^{n-1}$.

Note that according to Lemma 14.9, if $U$ is a proper round ball in $S$, then $d$ is one-to-one in restriction to $\bar{U}$ and $d(\bar{U})$ is a closed round ball of $\mathbb{S}^{n-1}$.

Definition 14.11. We will denote by $\mathcal{B}(S)$ the set of all round balls in $S$, and by $\mathcal{B}_{0}(S)$ the set of proper round balls.

The sets $\mathcal{B}(S)$ and $\mathcal{B}_{0}(S)$ are naturally ordered by the inclusion. For every element $U$ of $\mathcal{B}_{0}(S)$, we denote by $W(U)$ the subset of $\mathcal{B}_{0}(S)$ made of the proper round balls $U^{\prime}$ such that $\overline{U^{\prime}} \subset U$. Given two elements $U, V$ of $\mathcal{B}_{0}(S)$ such that $\bar{U} \subset V$, we denote by $W(U, V)$ the set of all proper round balls $U^{\prime}$ in $S$ such that $\bar{U} \subset U^{\prime}$ and $\overline{U^{\prime}} \subset V$. The sets $W(U, V)$ generate a topology on $\mathcal{B}_{0}(S)$ that we call the Alexandrov topology.

We already observed that the de Sitter space $\mathbb{D S}_{n}$, as a set, is canonically identified with the space $\mathcal{B}_{0}\left(\mathbb{S}_{+}^{n-1}\right)=\mathcal{B}\left(\mathbb{S}_{+}^{n-1}\right)$ of all open round balls in the sphere $\mathbb{S}_{+}^{n-1}$ (see $\S 14.2)$.

LEMma 14.12. The canonical identification between $\mathbb{D S}_{n}$ and $\mathcal{B}_{0}\left(\mathbb{S}_{+}^{n-1}\right)$ is an homeomorphim, once $\mathcal{B}_{0}\left(\mathbb{S}_{+}^{n-1}\right)$ is endowed with the Alexandrov topology.

Proof. Let $U, V$ be two elements in $\mathcal{B}_{0}\left(\mathbb{S}_{+}^{n-1}\right)$ such that $U \subset V$. Let $x, y$ be the points of $\mathbb{D S}_{n}$ corresponding respectively to $U$ and $V$. Recall that this means that $U$ (resp. $V$ ) is the set of future extremities of timelike geodesics starting at $x$ (resp. $y$ ). Hence $U \subset V$ implies $J^{+}(x) \subset I^{+}(y)$, or equivalently $p \in I^{+}(q)$. Now observe that the set $W(U, V) \subset \mathcal{B}_{0}\left(\mathbb{S}_{+}^{n-1}\right)$ corresponds in $\mathbb{D S}_{n}$ to the set of all points $z$ such that $J^{+}(x) \subset I^{+}(z)$ and $J^{+}(z) \subset I^{+}(y)$, or equivalently, $z \in I^{+}(y) \cap I^{-}(x)$. But since $\mathbb{D S}_{n}$ is strongly causal, the topology on $\mathbb{D S}_{n}$ generated by sets of the type $I^{+}(y) \cap I^{-}(x)$ is the same as the manifold topology. The lemma follows.

Proposition 14.13. The set $\mathcal{B}_{0}(S)$, equipped with the Alexandrov topology, is a manifold.

Sketch of proof. Compare our proof with [38, Proposition page 98, item (iii)]. The developing map $d: S \rightarrow \mathbb{S}^{n-1}$ induces a map $d: \mathcal{B}_{0}(S) \rightarrow \mathcal{B}_{0}\left(\mathbb{S}^{n-1}\right)$. The composition of this map with the identification between $\mathcal{B}_{0}\left(\mathbb{S}_{+}^{n-1}\right)$ with $\mathbb{D S}_{n}$ defines a natural map $\mathcal{D}^{+}: \mathcal{B}_{0}(S) \rightarrow \mathbb{D S}_{n}$. For any element $U$ of $\mathcal{B}_{0}(S)$, the restriction of $\mathcal{D}^{+}$to $W(U)$ is a homeomorphism onto its image, which is the future $I^{+}(x)$ of the point $x$ such that $\partial I^{+}(x)=d(U)$. It follows that the $W(U)$ 's (where $U$ ranges over $\mathcal{B}_{0}(S)$ ) are charts on $\mathcal{B}_{0}(S)$ homeomorphic to $\mathbb{R}^{n}$.

Let us prove the Hausdorff separation property: let $U_{1}, U_{2}$ be elements of $\mathcal{B}_{0}(S)$ such that every neighborhood of $U_{1}$ intersects every neighborhood of $U_{2}$. Let $U_{1}^{\prime}, U_{2}^{\prime}$ be other elements of $\mathcal{B}_{0}(S)$ such that $\bar{U}_{1} \subset U_{1}^{\prime}$ and $\bar{U}_{2} \subset U_{2}^{\prime}$. Then, the neighborhoods $W\left(U_{1}^{\prime}\right)$ and $W\left(U_{2}^{\prime}\right)$ have non-trivial intersection since the first contains $U_{1}$ and the second contains $U_{2}$. Let $V$ be a common element. The round ball $V$ is contained in $U_{1}^{\prime} \cap U_{2}^{\prime}$. This last intersection is not empty. According to Lemma 14.8, the image by $D$ of $U_{1}^{\prime} \cap U_{2}^{\prime}$ is $D\left(U_{1}^{\prime}\right) \cap D\left(U_{2}^{\prime}\right)$. It follows that the restriction of $D$ to the union $U_{1}^{\prime} \cup U_{2}^{\prime}$ is injective. Therefore, the restriction of $\mathcal{D}^{+}$to $W\left(U_{1}^{\prime}\right) \cup W\left(U_{2}^{\prime}\right)$ is a homeomorphism, and $\mathcal{D}^{+}\left(W\left(U_{1}^{\prime}\right) \cup W\left(U_{2}^{\prime}\right)\right)=I^{+}\left(\mathcal{D}^{+}\left(U_{1}^{\prime}\right)\right) \cup I^{+}\left(\mathcal{D}^{+}\left(U_{2}^{\prime}\right)\right)$. Since the Hausdorff property holds in $I^{+}\left(\mathcal{D}^{+}\left(U_{1}^{\prime}\right)\right) \cup I^{+}\left(\mathcal{D}^{+}\left(U_{2}^{\prime}\right)\right)$, we conclude that $U_{1}=U_{2}$. 
The fact that $\mathcal{B}_{0}(S)$ is second countable is not really relevant to our purpose, and its proof is left to the reader.

The map $\mathcal{D}^{+}: \mathcal{B}_{0}(S) \rightarrow \mathbb{D S}_{n}$ (obtained as the composition of the developing map $d: \mathcal{B}_{0}(S) \rightarrow \mathcal{B}_{0}\left(\mathbb{S}^{n-1}\right)$ and the identification of $\mathcal{B}_{0}\left(\mathbb{S}^{n-1}\right)$ with $\left.\mathbb{D} \mathbb{S}_{n}\right)$ is a local homeomorphism (see Lemma 14.12). Hence, we can consider the pull-back by $\mathcal{D}^{+}$of the de Sitter metric on $\mathcal{B}_{0}(S)$. This is a locally de Sitter lorentzian metric on $\mathcal{B}_{0}(S)$.

Definition 14.14. We will denote by $\mathcal{B}_{0}^{+}(S)$ the manifold $\mathcal{B}_{0}(S)$ equipped with the pull-back by $\mathcal{D}^{+}$of the de Sitter metric.

REMARK 14.15. It is clear from our definitions that the lorentzian manifold $\mathcal{B}_{0}^{+}(S)$ is future complete. It is also that $\mathcal{B}_{0}^{+}(S)$ is asymptotically simple, i.e. that every inextendible future oriented null geodesic ray is complete. It follows that $\mathcal{B}_{0}^{ \pm}(S)$ is globally hyperbolic (see Proposition 2.1 in [4]).

Proposition 14.16. In the case where $d$ is one-to-one, the lorentzian manifold $\mathcal{B}_{0}^{+}(S)$ defined in this paragraph is isometric to the domain $\mathcal{B}_{0}^{+}(d(S))$ defined in $\S 14.3$.

Proof. This follows from the constructions and from remark 14.7. The isometry is given by the map $\mathcal{D}^{+}$.

REMARK 14.17. If $d^{\prime}: S \rightarrow \mathbb{S}_{+}^{n-1}$ is another developing map, then $d^{\prime}=\phi \circ d$ where $\phi$ is an element of the Möbius group $O(1, n-1)$ (in particular, $\phi$ maps round balls on round balls). It follows that, up to isometry, the dS standard spacetime $\mathcal{B}_{0}^{+}(S)$ does not depend on the choice of $d$.

A similar construction (where the sphere $\mathbb{S}_{+}^{n-1}$ is replaced by the sphere $\mathbb{S}_{-}^{n-1}$ ) yields a past complete lorentzian manifold $\mathcal{B}_{0}^{-}(S)$.

Definition 14.18. A future (resp. past) complete $d S$ standard spacetime is a lorentzian manifold of the type $\mathcal{B}_{0}^{+}(S)$ (resp. $\mathcal{B}_{0}^{-}(S)$ ) where $S$ is a simply connected Möbius manifold.

If $S$ is conformally equivalent to a finite quotient of the sphere $\mathbb{S}^{n}$, then $S$ and $\mathcal{B}_{0}^{ \pm}(S)$ are said to be elliptic. If $S$ is conformally equivalent to the sphere $\mathbb{S}^{n}$ minus a single point, then $S$ and $\mathcal{B}_{0}^{ \pm}(S)$ are said to be parabolic. If $S$ is neither elliptic nor parabolic, then $S$ and $\mathcal{B}_{0}^{ \pm}(S)$ are said to be hyperbolic.

REMARK 14.19. Elliptic standard dS spacetimes are finite quotients of the de Sitter space itself. Up to isometry, there is only one future complete (resp. past complete) parabolic standard spacetime, which can be described as the future (resp. past) in $\mathbb{D S}_{n}$ of a point in the conformal boundary $\mathbb{S}_{-}^{n-1}$ (resp. $\mathbb{S}_{+}^{n-1}$ ).

14.5. Canonical neighbourhood and canonical domain of a point. Let $S$ a simply connected Möbius manifold of dimension $n-1$. Let $d: S \rightarrow \mathbb{S}_{+}^{n-1}$ be a developing map. In general, the $\mathrm{dS}$ standard spacetime $\mathcal{B}_{0}^{+}(S)$ does not admit any global isometric embedding in $\mathbb{D S}_{n}$. Nevertheless, for many purposes we will not need to study the geometry of the whole spacetime $\mathcal{B}_{0}^{+}(S)$, but only the geometry of some regions of $\mathcal{B}_{0}^{+}(S)$ (typically the past of a point in $\mathcal{B}_{0}^{+}(S)$ ). The purpose of this paragraph is to define some "big" regions of $\mathcal{B}_{0}^{+}(S)$ which admit some isometric embeddings in $\mathbb{D S}_{n}$.

Definition 14.20. For $x \in S$, we denote by $\mathcal{U}(x)$ the union of all the open round balls containing $x$. The set $\mathcal{U}(x)$ is called the canonical neighborhood of $x$ in $S$. 
Using Lemma 14.8 it is easy to prove the following proposition (see also [38, Proposition 4.1]).

Proposition 14.21. The restriction of $d$ to any canonical neighborhood is oneto-one. $\mathrm{C}$

Putting together Propositions 14.21 and 14.16, we get:

COROLlary 14.22. For every $x \in S$, the $d S$ standard spacetime $\mathcal{B}_{0}^{+}(\mathcal{U}(x))$ is isometric to the $d S$ standard spacetime $\mathcal{B}_{0}^{+}(d(\mathcal{U}(x))$ ) (associated to the open domain $d(\mathcal{U}(x))$ of $\mathbb{S}_{+}^{n-1}$. In particular, $\mathcal{B}_{0}^{+}(\mathcal{U}(x))$ is globally isometric to an opain domain in $\mathbb{D S}_{n}$.

Moreover, the past of a point can always be seen in a domain of the form $\mathcal{B}_{0}^{+}(\mathcal{U}(x))$.

Proposition 14.23. Let $U$ be an element of $\mathcal{B}_{0}^{+}(S)$ (i.e. a proper round ball in $S)$. Let $x \in U \subset S$. Then the canonical domain $\mathcal{B}_{0}^{+}(\mathcal{U}(x))$ contains the past of $U$ in $\mathcal{B}_{0}^{+}(S)$.

Proof. Recall that a round ball $V$ is in the past of $U$ in $\mathcal{B}_{0}^{+}(S)$ if and only if $V$ contains $U$. So, if $V$ is in the past of $U$, then $x \in V$; hence, $V \in \mathcal{B}_{0}^{+}(\mathcal{U}(x))$.

14.6. Another definition of dS standard spacetimes. The construction of dS standard spacetimes detailed in the previous paragraph is quite different from those given by Scannell in [45]. We will now explain Scannell's construction.

Let $S$ be a hyperbolic simply connected Möbius manifold of dimension $n-1$, and $d: S \rightarrow \mathbb{S}_{+}^{n-1}$ be a developing map. Let $\mathcal{B}_{\max }(S)$ be the set of maximal open round balls in $S$, i.e. the maximal elements of $\mathcal{B}(S)$. For every element $U$ of $\mathcal{B}_{\max }(S)$, let $\bar{U}$ be the the closure of $U$ in $S$, let $\overline{d(U)}$ be the closure of $d(U)$ in $\mathbb{S}^{n-1}$, and let $\Lambda_{S}(U)$ be the complement of $d(\bar{U})$ in $\overline{d(U)}$. Observe that $\Lambda_{S}(U)$ is closed in $\mathbb{S}^{n-1}$. The closed set $\overline{d(U)}$ is conformally equivalent to the compactified hyperbolic space $\mathbb{H}^{n-1} \cup \partial \mathbb{H}^{n-1}$. We may therefore transfer the usual notion of hyperbolic convex hull to $\overline{d(U)}$, and define the convex hull $\hat{C}(U)$ of $\Lambda_{S}(U)$ in $\overline{d(U)}$. Let $C(U)=d^{-1}(\widehat{C}(U)) \cap U$ (note that $C(U)=\emptyset$ if and only if $\Lambda_{S}(U)$ has less than two points). A key point in the construction is the following fact ([38, Theorem 4.4] or [45, Proposition 4.1]).

FACT. For every $x$ in $S$ there exists a unique element $U(x)$ of $\mathcal{B}_{\text {max }}(S)$ such that $x$ belongs to $C(U(x))$.

REMARK 14.24. This fact allows one to define a stratification of the Möbius manifold $S$ : for every $x \in S$, the stratum of $x$ is the set $C(U(x))$. This stratification - which was defined by Thurston in some particular case (unpublished), and later by Apanasov and Kulkarni-Pinkall in the general case (see [38]) — is called the canonical stratification of $S$.

Following Scannell (see [45, page 8]), we will now define a local homeomorphism $\mathbf{D}^{+}: S \times(0,+\infty) \rightarrow \mathbb{D S}_{n}$. We use the identification between $\mathbb{D S}_{n}$ and the set of round balls in $\mathbb{S}_{+}^{n-1}$ : for every $x$ in $S$, we see the round ball $U(x)$ as a point in $\mathbb{D S}_{n}$. Let $c_{x}:[0,+\infty) \rightarrow \mathbb{D S}_{n}$ be the unique unit speed future oriented timelike geodesic such that $c_{x}(0)=U(x)$ and $c_{x}(t) \rightarrow x$ when $t \rightarrow \infty$. We define $\mathbf{D}^{+}(x, t)$ as the point $c_{x}(t)$ in $\mathbb{D S}_{n}$. Scannell proved that this map is a local homeomorphism. Then we can define the future complete $d S$ standard spacetime $\mathbf{B}^{+}(S)$ associated with $S$ as the manifold $S \times(0,+\infty)$ equipped with the pull-back by $\mathbf{D}^{+}$of the de Sitter metric. 
We will see later (remark 15.9) that this definition of dS standard spacetimes coincides with the definition given in $\S 14.4$ (more precisely, the locally de Sitter manifolds $\mathbf{B}^{+}(S)$ and $\mathcal{B}_{0}^{+}(S)$ are isometric). At this point, it should be clear to the reader that there exists an isometric embedding $f: \mathbf{B}^{+}(S) \hookrightarrow \mathcal{B}_{0}^{+}(S)$ such that $\mathbf{D}^{+}=\mathcal{D}^{+} \circ f$.

\subsection{MGHC de Sitter spacetimes and dS standard spacetimes.}

ThEOREM 14.25 (Scannell). Every MGHC dS-spacetime is the quotient of a dS standard spacetime by a torsion-free discrete subgroup of isometries.

Proof. See [45] (and remark 15.9 which shows that Scannell's definition of dS standard spacetimes is equivalent to Kulkarni-Pinkall's definition).

15. Cosmological time and horizons of dS regular domains. All along this section, we consider a simply connected Möbius manifold $S$ of dimension $n-1$, and the associated (future complete) dS standard spacetime $\mathcal{B}_{0}^{+}(S)$. We assume that $S$ is hyperbolic.

Recall that $\mathcal{B}_{0}^{+}(S)$ is defined as follows. One chooses a developing map $d: S \rightarrow$ $\mathbb{S}_{+}^{n-1} \simeq \mathbb{S}^{n-1}$. One considers the space $\mathcal{B}_{0}\left(\mathbb{S}_{+}^{n-1}\right)$. This map induces a local homeomorphism $d: \mathcal{B}_{0}(S) \rightarrow \mathcal{B}_{0}\left(\mathbb{S}_{+}^{n-1}\right)$. The composition of this local homeomorphism with the identification between $\mathbb{D S}_{n}$ with $\mathcal{B}_{0}\left(\mathbb{S}_{+}^{n-1}\right)$ defines a local homeomorphism $\mathcal{D}^{+}: \mathcal{B}_{0}(S) \rightarrow \mathbb{D S}_{n}$. The dS standard spacetime $\mathcal{B}_{0}^{+}(S)$ is, by definition, the manifold $\mathcal{B}_{0}(S)$ equipped with the pull back by $\mathcal{D}^{+}$of the lorentzian metric of $\mathbb{D S}_{n}$. So, by construction, $\mathcal{D}^{+}$defines a locally isometric developing map of $\mathcal{B}_{0}^{+}(S)$ in $\mathbb{D S}_{n}$.

The purpose of this section is to get some information on the cosmological time of $\mathcal{B}_{0}^{+}(S)$. Just as in the AdS setting, this will lead us to study the support hyperplanes of the past horizon $\mathcal{H}^{-}(S)$ of $\mathcal{B}_{0}^{+}(S)$. Of course, a similar study could be carried out for the dS standard spacetime $\mathcal{B}_{0}^{-}(S)$.

\subsection{Cosmological time.}

Proposition 15.1. The dS standard spacetime $\mathcal{B}_{0}^{+}(S)$ has a regular cosmological time.

Proof. Recall that we have assumed that $S$ is hyperbolic; this will play a crucial role here. We denote by $\tau$ the cosmological time of $\mathcal{B}_{0}^{+}(S)$.

Let $x \in \mathcal{B}_{0}^{+}(S)$. We want to prove that $\tau(x)$ is finite. We argue by contradiction. If $\tau(x)=+\infty$, then, for every $n \in \mathbb{N}$, we can find a past directed causal curve $c_{n}:[0,1] \rightarrow \mathcal{B}_{0}^{+}(S)$ such that $c_{n}(0)=x$ and such that the length of $c_{n}$ is at least $n$. For every $n$, let $x_{n}:=c_{n}(1)$. Let $z:=\mathcal{D}^{+}(x)$. For every $n \in \mathbb{N}$, let $\gamma_{n}:=\mathcal{D}^{+} \circ c_{n}$ and $z_{n}:=\mathcal{D}^{+}\left(x_{n}\right)=\gamma_{n}(1)$. Then $\left(\gamma_{n}\right)_{n \in \mathbb{N}}$ is a sequence of past directed compact causal curves in $\mathbb{D S}_{n}$, all having the same final extremity $z$, and such that the length of $\gamma_{n}$ tends to $\infty$ when $n \rightarrow \infty$. It follows that, up to extracting a subsequence, the sequence $\left(z_{n}\right)_{n \in \mathbb{N}}$ converges to a point $\bar{z} \in \mathbb{S}_{-}^{n-1}$. Now, recall that $\left(x_{n}\right)_{n \in \mathbb{N}}$ is a sequence of points in $\mathcal{B}_{0}^{+}(S)$, that is, a sequence of proper round balls in $S$. Let $\bar{x}$ be the liminf of these balls, i.e. $\bar{x}=\bigcup_{p \in \mathbb{N}} \bigcap_{n>p} x_{n}$. Note that $d$ is one-to-one in restriction to $\bar{x}$ (since it is one-to-one in restriction to each $x_{i}$ ). For every $n$, the point $z_{n}$ can be seen as a ball in $\mathbb{S}_{+}^{n-1}$ (using the identification of $\mathbb{D S}_{n}$ with the space of round balls in $\left.\mathbb{S}_{+}^{n-1}\right)$. If we see $x_{n}$ as a ball in $S$ and $z_{n}$ as a ball in $\mathbb{S}_{+}^{n-1}$, then we have $z_{n}=d\left(x_{n}\right)$. Hence, $d(\bar{x})$ is the liminf of the sequence of balls $\left(z_{n}\right)_{n \in \mathbb{N}}$. Since $z_{n} \rightarrow \bar{z} \in \mathbb{S}_{-}^{n-1}$, it follows that $d(\bar{x})$ is the complement of a single point in $\mathbb{S}_{+}^{n-1}$. According to Lemma 14.9, this implies that the boundary of the ball $\bar{x}$ in $S$ is either 
empty, or a single point. In the former case, we have $y=S$, hence $S$ is parabolic, and this contradicts our hypothesis on $S$. In the latter case, the restriction of $d$ to the closure $\bar{y}$ is a homeomorphism onto $\mathbb{S}_{+}^{n-1}$; it follows that $S$ is elliptic, and this also contradicts our hypothesis. So $\tau(x)$ is finite.

Now, we consider an inextendible past oriented causal curve $c:[0, T) \rightarrow \mathcal{B}_{0}^{+}(S)$. We have to prove that $\tau(c(t)) \rightarrow 0$ when $t \rightarrow T$. Let $x:=c(0)$. On the one hand, for every $t \in[0, T)$, the quantity $\tau(c(t))$ does not depend on the whole spacetime $\mathcal{B}_{0}^{+}(S)$, but only on the past $J^{-}(x)$ of $x$ in $\mathcal{B}_{0}^{+}(S)$. On the other hand, the set $J^{-}(x)$ is contained in the domain $\mathcal{B}_{0}^{+}(\mathcal{U}(x))$ (Proposition 14.23). As a consequence, in our problem, we can replace the cosmological time $\tau$ of the dS standard spacetime $\mathcal{B}_{0}^{+}(S)$ by the cosmological time $\check{\tau}$ of standard spacetime $\mathcal{B}_{0}^{+}(\mathcal{U}(x)) \subset \mathcal{B}_{0}^{+}(S)$. But the standard spacetime $\mathcal{B}_{0}^{+}(\mathcal{U}(x))$ is isometric to a causally convex domain of $\mathbb{D S}_{n}$ (corollary 14.22 and remark 14.4). It follows easily that $\check{\tau}(c(t)) \rightarrow 0$ when $t \rightarrow T$. Therefore $\tau(c(t)) \rightarrow 0$ when $t \rightarrow T$.

REMARK 15.2.

1. Since MGHC de Sitter spacetimes are quotients of standard spacetimes by Theorem 14.25, and since cosmological time functions are preserved by isometries, it is an immediate corollary of Proposition 15.1 that MGHC hyperbolic standard spacetimes have regular cosmological time.

In [4, Theorem 3.1] it is shown that for a class of MGHC spacetimes (spacetimes of de Sitter type), satisfying the strong energy condition with positive cosmological constant, assuming that the future conformal boundary has an infinite fundamental group implies that the spacetime is past incomplete.

This result and our Proposition 15.1 have quite similar flavor. The result in [4] is more general since MGHC spacetimes of de Sitter type do not have in general constant curvature. On the other hand, the conclusion of Proposition 8.1 is stronger, since a spacetime may be past incomplete without having a regular cosmological time.

2. Elliptic and parabolic dS standard spacetimes do not have regular cosmological time. The cosmological time in these spacetimes is everywhere infinite.

Of course, there are analogs of Theorem 2.3 and Proposition 15.1, concerning the reverse cosmological time in past complete dS standard spacetimes.

15.2. Past horizon. As in the AdS case, one can define a notion of past horizon for future complete $\mathrm{dS}$ standard spacetimes. Recall that $\mathcal{B}_{0}(S)$ is the set of proper open round balls in $S$, whereas $\mathcal{B}(S)$ is the set of all round balls in $S$ (see $\S 14.4$ ).

Definition 15.3. The past horizon of the future complete regular domain $\mathcal{B}_{0}(S)$ is the set $\mathcal{H}^{-}(S):=\mathcal{B}(S) \backslash \mathcal{B}_{0}(S)$.

REMARK 15.4 .

1. The arguments of Proposition 14.13 can be easily adapted, leading to the conclusion that the set $\mathcal{B}(S)$ admits a topology for which it is a manifold with boundary (the boundary being precisely the past horizon $\mathcal{H}^{-}(S)=$ $\left.\mathcal{B}(S) \backslash \mathcal{B}_{0}(S)\right)$. Moreover, the developing map $\mathcal{D}^{+}: \mathcal{B}_{0}(S) \rightarrow \mathbb{D S}_{n}$ extends to a local homeomorphism from $\mathcal{B}(S)$ into $\mathbb{D S}_{n}$ that we still denote by $\mathcal{D}^{ \pm}$.

2. Every round ball in $S$ is the increasing union of the elements of a oneparameter family of proper round balls. It follows that any past-extendible causal curve $c$ in $\mathcal{B}_{0}^{+}(S)$ admits a limit point in the horizon $\mathcal{H}^{-}(S)$; we call this point the initial extremity of the curve $c$. Conversely, any point $p \in \mathcal{H}^{-}(S)$ 
is the initial extremity of a past-inextendible timelike curve in $\mathcal{B}_{0}^{-}(S)$ (which can actually be chosen to be geodesic).

3. Recall that, in the particular case where $S$ is an open domain in $\mathbb{S}_{+}^{n-1}$, the dS standard spacetime $\mathcal{B}_{0}^{+}(S)$ can be seen as an open domain domain in the de Sitter space $\mathbb{D S}_{n}$. Using item (1), it is easy to see that, in this particular case, the past horizon $\mathcal{H}^{-}(S)$ is just the topological boundary in $\mathbb{D S}_{n}$ of the open domain $\mathcal{B}_{0}^{+}(S)$.

As noticed above, the past horizon $\mathcal{H}^{-}(S)$ admits a simple description in the particular case where the developing map $d$ is one-to-one. Lemma 15.5 shows that, as far as only "semi-local" properties are involved of $\mathcal{H}^{-}(S)$, one can always reduce to this particular case. We recall that every point $q \in S$ admits a "nice" neighbourhood $\mathcal{U}(q)$ in $S$ which is isometric to an open domain in $\mathbb{S}_{+}^{n-1}$.

LEMMA 15.5. Let $p$ be a point in $\mathcal{H}^{-}(S)$. Let $c$ be a future complete timelike geodesic with initial extremity $p$. Let $q$ be the future extremity of $c$ in $\mathbb{S}_{n-1}^{+}$. For every element $x$ in $c$, let $\mathcal{H}_{x}^{-}(S)$ be the intersection of $\mathcal{H}^{-}(S)$ with the closure of $I^{-}(x)$ in $\mathcal{B}^{+}(S)$. Similarly, let $\mathcal{H}_{x}^{-}(\mathcal{U}(q))$ be the intersection of $\mathcal{H}^{-}(\mathcal{U}(q))$ with the closure of $I^{-}(x)$ in $\mathcal{B}^{+}(\mathcal{U}(q))$. Then $\mathcal{H}_{x}^{-}(S)$ is an open neighborhood of $p$ in $\mathcal{H}^{-}(S)$ and coincides with $\mathcal{H}_{x}^{-}(\mathcal{U}(q))$.

Proof. This is an immediate corollary of Proposition 14.23.

Let us assume that $S$ is a domain in the sphere $\mathbb{S}_{+}^{n-1}$. Recall that, under this assumption, the dS standard spacetime $\mathcal{B}_{0}^{+}(S)$ is a domain in $\mathbb{D S}_{n}$, and the past horizon $\mathcal{H}^{-}(S)$ is just the boundary of $\mathcal{B}_{0}^{+}(S)$ in $\mathbb{D S}_{n}$. Also recall that $\mathcal{B}_{0}^{+}(S)$ is defined as a connected component of the intersection of the convex set $\Omega^{+}(S)$ with $\mathbb{D S}_{n}$ (see $\S 14.3$ ). In particular, $\mathcal{H}^{-}(S)$ is a locally convex hypersurface in $\mathbb{S}\left(\mathbb{R}^{n+1}\right)$. This allows us to define the notion of support planes of $\mathcal{H}^{-}(S)$ (which are projective hyperplanes in $\mathbb{S}\left(\mathbb{R}^{n+1}\right)$. Note that, just as in AdS case, if $H$ is a support hyperplane of $\mathcal{H}^{-}(S)$, then the totally geodesic hypersurface $H \cap \mathbb{D S}_{n}$ is a spacelike or degenerate. The following statement is the analog of Proposition 11.2 in the AdS case.

Proposition 15.6. Assume that $S$ is a domain in $\mathbb{S}_{+}^{n-1}$. Let $p$ a point of $\mathcal{H}^{-}(S)$. Let $C(p) \subset T_{p} \mathbb{D S}_{n}$ be the set of the future directed unit tangent vectors orthogonal to the support hyperplanes of $\mathcal{H}^{-}(S)$ at $p$. Then:

1. the set $C(p)$ is the convex hull of its lightlike elements;

2. if $c$ is a future complete geodesic ray starting at $p$ whose tangent vector at $p$ is a lightlike element of $C(p)$, then the future endpoint of $c$ is in $\Lambda$ (recall that $\Lambda$ is the boundary of $S$ in $\left.\mathbb{S}_{+}^{n-1}\right)$.

Proof. The proof is very similar to those of Proposition 11.2; the only differences are the following.

- We work with the convex set $\Omega^{+}(S)$ instead of the convex set $E(\Lambda)$.

- The point $q$ now belongs to $\mathbb{H}_{+}^{n} \cup \mathbb{S}_{+}^{n-1}$ (instead of $\mathbb{A D S}_{n} \cup \partial \mathbb{A D S}_{n}$ in the AdS case).

- The causal vector $v_{q}$ is lighlike if and only if $q \in \mathbb{S}_{+}^{n+1}$.

- The proof of item (2) is slightly easier than in the dS case: since the quadratic form $Q_{1, n}$ has signature $(1, n)$, one gets that the subspace spanned by the $\hat{q}_{i}$ 's is 1-dimensional (instead of 2-dimensional in the AdS case); it follows immediately that all the $q_{i}$ 's are equal to $q$, and thus, that $q$ is in $\Lambda$. 
15.3. Retraction onto the horizon. We now study the realizing geodesics in $\mathcal{B}_{0}^{+}(S)$. Let $x \in \mathcal{B}_{0}^{+}(S)$. Recall that a future directed timelike geodesic ray $c:(0,1] \rightarrow$ $\mathcal{B}_{0}^{+}(S)$ such that $c(1)=x$ is a realizing geodesic for $x$ if $\tau(x)$ is equal to the length of $c$. Clearly, realizing geodesic rays for $x$ are contained in the past of $x$. Therefore, for our problem, we may pick a point $q \in \mathbb{S}_{+}^{n-1}$ which is the future endpoint of a timelike geodesic passing through $x$, and replace the $\mathrm{dS}$ standard spacetime $\mathcal{B}_{0}^{+}(S)$ by the $\mathrm{dS}$ standard spacetime $\mathcal{B}_{0}^{+}(\mathcal{U}(q)$ ) (Proposition 14.23). In other words, as far as realizing geodesic rays for $x$ are concerned, we may assume without loss of generality that $S$ is an open domain in the sphere $\mathbb{S}_{+}^{n-1}$.

Proposition 15.7. For every $x \in \mathcal{B}_{0}^{+}(S)$, there is a unique realizing geodesic for $x$ in $\mathcal{B}_{0}^{+}(S)$.

Proof. Recall that we assume without loss of generality that $S$ is a domain in $\mathbb{S}_{+}^{n-1}$. Hence, the dS standard spacetime $\mathcal{B}_{0}^{+}(S)$ is a connected component of the intersection of the convex set $\Omega^{+}(S)$ with $\mathbb{D S}_{n}$, and $\mathcal{H}^{-}(S)$ is the boundary of $\mathcal{B}_{0}^{+}(S)$ in $\mathbb{D S}_{n}$. Initial extremities of realizing geodesics for $x$ are points $z$ in $\mathcal{H}^{-}(S)$ such that $d(x, z)=\tau(x)$, where $d(x, z)$ is the length of a past oriented timelike geodesic in $\mathbb{D S}_{n}$ starting from $x$ and ending to $z$. For each $\tau$, the set $\left\{z \in \mathbb{D S}_{n} \mid d(x, z) \geq \tau\right\}$ is the intersection of $\mathbb{D S}_{n}$ with a solid ellipsoid $\mathcal{E}_{\tau}$ in $\mathbb{S}\left(\mathbb{R}^{n}\right)$ tangent to the sphere $\mathbb{S}_{-}^{n-1}$ along a round subsphere. If $\tau<\tau^{\prime}$, then $\mathcal{E}_{\tau^{\prime}} \subset \operatorname{int} \mathcal{E}_{\tau}$, leading to the definition:

$$
\tau(x)=\sup \left\{\tau \mid \mathcal{E}_{\tau} \cap \mathcal{H}_{-}(S) \neq \emptyset\right\} .
$$

Let $y, y^{\prime}$ be initial extremities of realizing geodesics for $x$ : they both belong to $\mathcal{E}_{\tau(x)} \cap$ $\bar{\Omega}^{+}(\Lambda)$. On one hand, the segment $\left[y, y^{\prime}\right]$ is contained in the interior of $\mathcal{E}_{\tau(x)}$ (since ellipsoids are strictly convex). On the other hand, according to Remark 14.2, the segment $\left[y, y^{\prime}\right]$ is contained in $\mathcal{B}^{+}(S)$. We obtain a contradiction, unless $y=y^{\prime}$ (see the proof of Proposition 11.5).

Proposition 15.8. Let $c:(0, T] \rightarrow \mathcal{B}_{0}^{-}(S)$ be a future oriented timelike geodesic whose initial extremity $p=\lim _{t \rightarrow 0} c(t)$ belongs to the past horizon $\mathcal{H}^{-}(\Lambda)$. Then the following assertions are equivalent:

1. the geodesic $c$ is tight,

2. there exists $t_{0} \in(0, T]$ such that $c\left(\left(0, t_{0}\right]\right)$ is a realizing geodesic for the point $c(t)$,

3. $c$ is orthogonal to a support hyperplane of $\Omega^{+}(S)$ at $p$.

Proof. The proof is entirely similar to those of Proposition 11.6, based on the strict convexity of the ellipsoids $\mathcal{E}_{\tau}$.

REMARK 15.9. According to Lemma 15.6, and since there is at least one realizing geodesic for each $x$ in $\mathcal{B}_{0}^{+}(S)$, Proposition 15.8 means precisely that the map $f: \mathbf{B}(S) \rightarrow \mathcal{B}_{0}^{+}(S)$ defined at the end of $\S 14.1$ is onto. Hence $f$ is an isometric identification between $\mathcal{B}_{0}^{+}(S)$ and $\mathbf{B}(S)$.

16. dS regular domains: curvature estimates for cosmological time level sets.

THEOREM 16.1. Let $\mathcal{B}_{0}^{+}(S)$ be a future complete $d S$ standard spacetime, and $\tau: \mathcal{B}_{0}^{+}(S) \rightarrow(0,+\infty)$ be the associated cosmological time function. Then, for every $a \in(0,+\infty)$, the generalized mean curvature of the level set $S_{a}=\tau^{-1}(a)$ admits the 
following estimates

$$
-\operatorname{coth}(a) \leq H_{S_{a}} \leq-\frac{1}{n-1} \operatorname{coth}(a)-\frac{n-2}{n-1} \tanh (a) .
$$

Proof. We use the same notations $x, p, c, v$ as in the proof of Theorem 12.1. The past of the geodesic $c: \mathbb{R} \rightarrow \mathcal{B}_{0}^{+}(S)$ contains the past in $\mathcal{B}_{0}^{+}(S)$ of a small neighbourhood $U$ of $x$. The restriction to $U$ of the function $\tau$ only depends on the past of $U$ in $\mathcal{B}_{0}^{+}(S)$. Hence the geometry of the hypersurface $S_{a}$ in $U$ (in particular the generalized mean curvature of $S_{a}$ at $p$ ) only depends on the past of $c$ in $\mathcal{B}_{0}^{+}(S)$. Together with Lemma 15.5, this allows us to restrict ourselves to the case where $S$ is an open domain in $\mathbb{S}_{+}^{n-1}$.

The proof is then formally completely similar to that of Theorem 12.1. The hypersurface $\mathbb{S}_{x}^{+}$is the set of the points of $\mathcal{B}_{0}^{+}(S)$ which are in the future of $p$, at distance exactly $a$ from $p$. Clearly, $\mathbb{S}_{x}^{+}$is in the future of $S_{a}$, and $x \in \mathbb{S}_{x}^{+}$. A simple computation shows that the mean curvature of $\mathbb{S}_{x}^{+}$is constant and equal to $-\operatorname{coth}(a)$.

In order to construct the hypersurface $\mathbb{S}_{x}^{-}$, we select a finite set $v_{1}, \ldots, v_{r}$ of lightlike elements of $C(p)$ such that $v \in \operatorname{Conv}\left(v_{1}, \ldots, v_{r}\right)$ (such a finite set does exist by item (1) of Proposition 15.6). For every $i$, we denote by $q_{i}$ the future endpoint of the lightlike geodesic ray whose tangent vector at $p$ is the vector $v_{i}$. Let $S^{\prime}=$ $\mathbb{S}_{+}^{n-1} \backslash\left\{q_{1}, \ldots, q_{r}\right\}$. Item (2) of Proposition 15.6 shows that $S^{\prime} \supset S$. The domain $\mathcal{B}_{0}^{+}\left(S^{\prime}\right) \subset \mathbb{D S}_{n}$ is a dS standard spacetime with regular cosmological time $\tau^{\prime}$. We define the hypersurface $\mathbb{S}_{x}^{-}$as the $a$-level set of the cosmological time $\tau^{\prime}$. Since $S^{\prime} \supset S$, the domain $\mathcal{B}_{0}^{+}\left(S^{\prime}\right)$ contains the domain $\mathcal{B}_{0}^{+}(S)$, and thus, $\mathbb{S}_{x}^{-}$is in the past of $S_{a}$.

So, we are left to compute the mean curvature of $\mathbb{S}_{x}^{-}$at $p$. For this purpose, we introduce the minimal projective subspace $F$ in $\mathbb{S}\left(\mathbb{R}^{n+1}\right)$ containing $q_{1}, \ldots, q_{r}$. We observe that $\mathbb{S}_{x}^{-}=\left(\tau^{\prime}\right)^{-1}(a)$ is the saturation under $G$ of the umbilical submanifold $S_{a} \cap F^{\perp}$, where $G$ is the group of isometries fixing $F$ pointwise. It follows that the mean curvature of $\mathbb{S}_{x}^{-}$is constant and equals:

$$
-\frac{d}{n-1} \operatorname{coth}(a)+\frac{n-1-d}{n-1} \tanh (a)
$$

for some $d \in\{1, \ldots, n-1\}$. Finally, one observes that this quantity is maximal when $d$ is minimal (i.e. when $d=1$ ). The theorem follows.

REMARK 16.2. The past barriers appearing in the proof are the CMC hypersurfaces presented in Example 2 of [42].

By reversing the time one obtains the following result.

THEOREM 16.3. Let $\mathcal{B}_{0}^{-}(S)$ be a past complete $d S_{n}$ regular domain, and $\widehat{\tau}$ : $\mathcal{B}_{0}^{-}(S) \rightarrow(0,+\infty)$ be the reverse cosmological time function associated to $\mathcal{B}_{0}^{-}(S)$. Then, for every $a \in(0,+\infty)$, the generalized mean curvature of the level set $\widehat{S_{a}}=$ $\widehat{\tau}^{-1}($ a) admits the following estimates:

$$
\frac{1}{n-1} \operatorname{coth}(a)+\frac{n-2}{n-1} \tanh (a) \leq H_{\widehat{S_{a}}} \leq \operatorname{coth}(a) .
$$

17. CMC time functions in de Sitter MGHC spacetimes. In this section we prove Proposition 1.10 and Theorem 1.11, and discuss CMC foliations in elliptic de Sitter spacetimes. The existence problem of CMC-times or CMC-foliations splits into several cases (essentially three) and subcases. 
17.1. The hyperbolic case. The proof of Theorem 1.11 is very similar to that of Theorem 1.4. The only difference is that, in the de Sitter case, the cosmological time function does not provide a sequence of future asymptotic barriers (except in dimension $2+1)$.

Proof of Theorem 1.11. Let $(M, g)$ be a past incomplete $n$-dimensional de Sitter MGHC spacetime. According to Theorem 14.25, $(M, g)$ is the quotient of a regular domain $\mathcal{B}_{0}^{+}(S)$ by a torsion-free discrete group $\Gamma \subset \operatorname{Isom}\left(\mathrm{dS}_{n}\right)$. The cosmological time $\tau: \mathcal{B}_{0}^{+}(S) \rightarrow(0,+\infty)$ is well-defined and regular.

For every $a \in[0,+\infty]$, let $S_{a}=\tau^{-1}(a)$ and $\Sigma_{a}$ be the projection of $S_{a}$ in $M \equiv$ $\Gamma \backslash \mathcal{B}_{0}^{+}(S)$. As every compact level set of a time function, $\Sigma_{a}$ is a topological Cauchy hypersurface in $M$ for every $a$. Theorem 16.1 implies that, for every $a \in(0,+\infty)$, the generalized mean curvature of $\Sigma_{a}$ satisfies

$$
-\operatorname{coth}(a) \leq H_{\Sigma_{a}} \leq-\frac{1}{n-1} \operatorname{coth}(a)-\frac{n-2}{n-1} \tanh (a) .
$$

Let $\left(a_{m}\right)_{m \in \mathbb{N}}$ be a decreasing sequence of positive real numbers such that $a_{m} \rightarrow 0$ when $m \rightarrow+\infty$. Observe that

$$
-\frac{1}{n-1} \operatorname{coth}\left(a_{m}\right)-\frac{n-2}{n-1} \tanh \left(a_{m}\right) \rightarrow-\infty \quad \text { when } m \rightarrow \infty .
$$

Hence $\left(\Sigma_{a_{m}}\right)_{m \in \mathbb{N}}$ is a sequence of past asymptotic $\alpha$-barrier in $M$ for $\alpha=-\infty$. Hence remark 4.10 implies that $M$ admits a partially defined CMC-time $\tau_{c m c}: U \rightarrow(-\infty, \beta)$ where $U$ is a neighbourhood of the past end of $M$.

17.1.1. The three-dimensional case. Assume $n=3$. Consider a sequence $\left(b_{m}\right)_{m \in \mathbb{N}}$ of increasing positive real numbers such that $b_{m} \rightarrow+\infty$ when $m \rightarrow+\infty$. For every $m \in \mathbb{N}$, one has

$$
-\operatorname{coth}\left(b_{m}\right)<-\frac{1}{2} \operatorname{coth}\left(b_{m}\right)-\frac{1}{2} \tanh \left(b_{m}\right)<-1
$$

and

$$
-\operatorname{coth}\left(b_{m}\right) \rightarrow-1 \quad \text { when } m \rightarrow \infty
$$

Hence $\left(\Sigma_{b_{m}}\right)_{m \in \mathbb{N}}$ is a sequence of future asymptotic $\beta$-barrier in $M$ for $\beta=-1$. Therefore, Theorem 4.3 implies that $M$ admits admits a globally defined CMC time function $\tau_{c m c}: M \rightarrow(-\infty,-1)$.

REMARK 17.1. This argument fails if $n>3$. The problem is that the quantity

$$
-\frac{1}{n-1} \operatorname{coth}(a)-\frac{n-2}{n-1} \tanh (a)
$$

becomes bigger than -1 when $a$ is large. See $\S 17.1 .3$ below.

17.1.2. The almost-fuchsian case. In the almost-fuchsian case there is an embedded Cauchy surface $\Sigma$ in $(M, g)$ with all principal eigenvalues $<-1$. Reversing the time if needed, we can assume that $M$ is future complete. Denote by $\Sigma_{t}$ the image of the hypersurface $\Sigma$ under the time $t$ map of the Gauss flow, i.e. obtained by pushing $\Sigma$ during a time $t$ along its normal geodesics. It is easy to describe in our context these hypersurfaces: let $\widetilde{\Sigma}$ be the universal covering of $\Sigma$ : the embedding $\Sigma \subset M$ lifts 
to an embedding $u: \widetilde{\Sigma} \rightarrow \mathcal{B}_{0}^{+}(S)$. For every $x$ in $\widetilde{\Sigma}$, there exists a unique element $u^{*}(x)$ of $\mathbb{H}_{+}^{n}$ such that the line $\mathbb{R} . u^{*}(x)$ is the $Q_{1, n}$-orthogonal of $\mathbb{R} . H(x)$ where $H(x)$ is the tangent projective hyperplane of $\widetilde{\Sigma}$ at $x$. In other words, we have two maps $u, u^{*}: \widetilde{\Sigma} \rightarrow \mathbb{R}^{1, n}$ such that, for every $x$ in $\widetilde{\Sigma}$,

- $Q_{1, n}(u(x))=1$,

- $Q_{1, n}\left(u^{*}(x)\right)=-1$,

- $\left\langle u(x) \mid u^{*}(x)\right\rangle=0$,

- for every tangent vector $\partial_{x}$ at $u(x)$ we have $\left\langle u^{*}(x) \mid \partial_{x} u\right\rangle=0$.

Then for every $x$ in $\widetilde{\Sigma}$ we have $\left\langle u(x) \mid \partial_{x} u^{*}\right\rangle=0$. The Weingarten operator for $\widetilde{\Sigma}$ is the linear operator $B$ such that $B\left(\partial_{x} u\right)=-\partial_{x} u^{*}$ for every tangent vector $\partial_{x}$.

The Gauss flow is described as follows: for every $t \geq 0$, let $u_{t}: \widetilde{\Sigma} \rightarrow \mathrm{dS}_{n} \subset \mathbb{R}^{1, n}$ defined by $u_{t}(x)=\cosh (t) u(x)+\sinh (t) u^{*}(x)$. Observe that since we have selected $u^{*}(x)$ in $\mathbb{H}_{+}^{n}$ the point $u_{t}(x)$ (for a fixed $x$ ) describes a future oriented geodesic ray starting from $u(x)$. The projection in $M$ of the image $\widetilde{\Sigma}_{t}$ of $u_{t}$ is the hypersurface $\Sigma_{t}$.

For a fixed $t$, the differential of $u_{t}$ evaluated on a tangent vector $\partial_{x}$ is $\cosh (t) \partial_{x} u+$ $\sinh (t) \partial_{x} u^{*}=(\cosh (t) I d-\sinh (t) B)\left(\partial_{x} u\right)$.

By assumption, the principal curvatures of $\Sigma$, i.e. the eigenvalues of $B$, are less than -1 . It follows that $u_{t}$ is an immersion for every $t \geq 0$ : the Gauss flow is defined for all positive $t$. Moreover, the differential of $u_{t}^{*}$ evaluated on $\partial_{x}$ is $(\sinh (t) I d-$ $\cosh (t) B)\left(\partial_{x} u\right)$. It follows that the Weingarten operator for $B_{t}$ is $-(\tanh (t) I d-$ $B)(I d-\tanh (t) B)^{-1}$. In particular, the mean curvature of $\Sigma_{t}$ is smaller than -1 for every $t \geq 0$, and tends to -1 when $t \rightarrow+\infty$.

Now, we claim that given an increasing sequence $\left(t_{m}\right)_{m \in \mathbb{N}}$ of real numbers such that $t_{m} \rightarrow \infty$ when $m \rightarrow \infty$, the sequence of hypersurfaces $\left(\Sigma_{t_{m}}\right)_{m \in \mathbb{N}}$ is a sequence of future asymptotic $\beta$-barrier in $M$ for $\beta=-1$. The only remaining point to check is that $\left(\Sigma_{t_{m}}\right)_{m \in \mathbb{N}}$ tends to the future end of $M$ when $m \rightarrow+\infty$. But this is clear: let $T_{0}$ be the minimal value of the cosmological time function on $\Sigma$. Then the cosmological time function restricted to $\Sigma_{t}$ is everywhere bigger than $T_{0}+t$. The claim follows.

Hence Theorem 4.3 implies that $M$ admits a globally defined CMC-time $\tau_{c m c}$ : $M \rightarrow(-\infty,-1)$.

REMARK 17.2. We define (future complete) fuchsian de Sitter spacetimes as MGHC de Sitter spacetimes $\left(M, g_{0}\right)=\mathcal{B}_{0}^{+}(S)$ where the Möbius manifold $S$ is a quotient $\Gamma \backslash U$ of a proper round ball $U$ in $\mathbb{S}_{+}^{n-1}$.

The metric of a Fuchsian spacetime is a warped product of the form $-d t^{2}+w(t)^{2} h$, where $h$ is independent of $t$. Any metric of this form admits a timelike homothety and is conformal to a static spacetime.

Observe that in particular the holonomy group $\Gamma$ is conjugate in $\mathrm{SO}_{0}(1, n)$ to a lattice of $\mathrm{SO}_{0}(1, n-1) ; \Gamma$ preserves a totally geodesic hypersurface $\mathbb{H}^{n-1}$ in $\mathbb{H}^{n}$.

We claim that $\left(M, g_{0}\right)$ is almost-fuchsian. To see this, consider a hypersurface $\Sigma$ dual to a hypersurface in $\mathbb{H}^{n}$ all the principal curvatures of which are very small (this last hypersurface can be obtained by taking the image of the totally geodesic hypersurface $\mathbb{H}^{n-1}$ under the time $t$ map of the Gauss flow for $t$ small).

If another Lorentz metric $g$ of dS type is a small deformation of the fuchsian metric $g_{0}$, then the hypersurface $\Sigma$ also has all its principal curvatures $<-1$ (with respect to $g$ ).

Remark 17.3. In dimension $2+1$, Theorem 1.11 can also be deduced from the existence of foliation of hyperbolic ends by surfaces with constant Gauss curvature (see $[15])$. 
17.1.3. A regular spacetime with no CMC time function. For every $n \geq$ 4 , there exists $n$-dimensional MGHC regular spacetimes that do not admit any CMC time function. Here is a construction of such a spacetime. Let $n \geq 4$ and choose as Möbius surface $S$ the complement in $\mathbb{S}_{+}^{n-1}$ of two points, say $p_{1}$ and $p_{2}$. Let $P_{1}$ and $P_{2}$ be the projective hyperplanes in $\mathbb{S}\left(\mathbb{R}^{n+1}\right)$ which are tangent to $\mathbb{S}_{+}^{n-1}$ respectively at $p_{1}$ and $p_{2}$. The intersection $Q=P_{1} \cap P_{2}$ is a spacelike totally geodesic subspace of dimension $n-2$ in $\mathbb{D S}_{n}$, homeomorphic to $\mathbb{S}^{n-2}$. The domain $\mathbb{B}_{0}^{+}(S)$ is by definition the intersection of the futures of $P_{1}$ and the future of $P_{2}$. It can be easily proved that the cosmological time function $\widetilde{\tau}$ of $\mathcal{B}_{0}^{+}(S)$ is just the lorentzian distance to the spacelike totally geodesic $(n-2)$-sphere $Q$. Using this, one can verify that, for every $a$, the level set $S_{a}=\widetilde{\tau}^{-1}(a)$ is a Cauchy hypersurface in $\mathbb{B}_{0}^{+}(S)$ which is homeomorphic to the $\mathbb{S}^{n-2} \times \mathbb{R}$, and has constant mean curvature equal to

$$
-\frac{1}{n-1} \operatorname{coth}(a)-\frac{n-2}{n-1} \tanh (a)
$$

(the calculation of the mean curvature is entirely similar to the estimates of the curvature of the hypersurface $\mathbb{S}^{+}$in the proofs of Theorem 12.1 and 16.1). Now, observe that the regular domain $\mathcal{B}_{0}^{+}(S)$ admits (regular) Cauchy compact quotients: if $\Gamma$ is a cyclic group generated by a hyperbolic element of $\mathrm{SO}_{0}(1, n)$ fixing the points $p_{1}$ and $p_{2}$, then $\Gamma$ acts properly discontinuously on $\mathbb{B}_{0}^{+}(S)$ and the projection $\Sigma_{a}$ of $S_{a}$ in the quotient $M:=\Gamma \backslash B_{0}^{+}(S)$ is a Cauchy hypersurface homeomorphic to $\mathbb{S}^{n-2} \times \mathbb{S}^{1}$. Moreover, for every $a$, the hypersurface $\Sigma$ has constant mean curvature equal to $-\frac{1}{n-1} \operatorname{coth}(a)-\frac{n-2}{n-1} \tanh (a)$. Hence $\mathcal{F}=\left\{\Sigma_{a}\right\}_{a \in(0,+\infty)}$ is a CMC foliation of $M$. But the mean curvature of the leaves of $\mathcal{F}$ is not monotonous (it increases for $a$ small, but decreases for $a$ large). In particular, $M$ does not admit any CMC time function (if there would exist a CMC time function, then the hypersurface $\Sigma_{a}$ would be a fiber of this CMC time function for every $a$, and thus, the mean curvature of $\Sigma_{a}$ would be a monotonous function of $a$ ).

This raises the following question.

QUESTIOn. Does every MGHC regular spacetime admit a global CMC foliation with compact leaves?

\subsection{The elliptic case.}

17.2.1. de Sitter space. We first consider the case of de Sitter space itself $\mathrm{dS}_{n}$. A key fact is that compact CMC hypersurfaces in $\mathrm{dS}_{n}$ are umbilical (see [43]; this is of course reminiscent of the Alexandrov rigidity Theorem which states that any compact CMC hypersurface in the Euclidean space is a round sphere). More precisely, they are the intersections between $\mathrm{dS}_{n}=\left\{Q_{1, n}=1\right\}$ and the affine spacelike hyperplanes of the Minkowski space $\mathbb{R}^{1, n}$. Such an hyperplane is defined as the set $H_{(t, v)}=\{x \mid\langle x \mid v\rangle=\sinh (t)\}$ where $v$ is a vector of norm -1 in the future cone of the Minkowski space, i.e. an element of the hyperbolic space $\mathbb{H}^{n}=\left\{Q_{1, n}=-1\right\}$, and $t$ a real number. Then, the intersection $S_{(t, v)}=H_{\left(t, v_{0}\right)} \cap \mathrm{dS}_{n}$ is an umbilical sphere, and every closed CMC surface in $\mathrm{dS}_{n}$ must be such an intersection. In other words, $\mathbb{H}^{n} \times \mathbb{R}$ is the space of umbilical spheres.

The mean curvature of $S_{(t, v)}$ is $-\tanh (t)$. It follows that if $S_{(t, v)}$ is in the future of $S_{\left(t^{\prime}, v^{\prime}\right)}$, then the mean curvature of the former is less than the mean curvature of the later. This phenomenon is actually valid locally.

LEMMA 17.4. Let $U$ be an open subset of $d S_{n}$ endowed with an umbilical foliation 
$\mathcal{F}$ with compact leaves. Then, the mean curvature function of $\mathcal{F}$ is decreasing. In particular, $d S_{n}$ has no CMC time.

Proof. By contradiction, assume that the mean curvature is somewhere increasing (or just non-decreasing). This will be true on an open $\mathcal{F}$-saturated set, we can thus assume that this holds on all $U$. Therefore, on $U$, we have a CMC time. By a well known property, any other compact CMC hypersurface in $U$ is a leaf of $\mathcal{F}$. This is obviously false: take $S$ a leaf of $\mathcal{F}$, and $S^{\prime}$ an umbilical hypersurface close to it, then $S^{\prime}$ will be contained in $U$, but is not necessarily a leaf of $\mathcal{F}$.

Observe that for a global foliation of $\mathrm{dS}_{n}$, leaves accumulate to the two boundary components, which can be thus seen as umbilical hypersurfaces, but with infinite curvature. More formally, the curvature of leaves decreases (with time) from $+\infty$ to $-\infty$.

We want to describe now CMC-foliations in $\mathrm{dS}_{n}$. The following Proposition gives a complete description.

Proposition 17.5. There is a 1-1 correspondance between CMC-foliations with compact leaves in $d S_{n}$ and inextendible timelike curves in $\mathbb{H}^{n} \times \mathbb{R}$ equipped with the lorentzian metric $d s_{\text {hyp }}^{2}-d t^{2}$ where $d s_{\text {hyp }}^{2}$ is the hyperbolic metric of $\mathbb{H}^{n}$.

Proof. Let $\mathcal{F}$ be a CMC-foliation with compact leaves. In order to simplify the proof, we assume that $\mathcal{F}$ is $C^{1}$, but see remark 17.7. The leaves are umbilical spheres $S_{(t, v)}$. Observe that since the leaves are disjoint one to the other, two different leaves must have different parameter $t$. By the Reeb stability Theorem (see [34]), since every leaf is a sphere, the foliation is trivial: there is a map $f: \mathrm{dS}_{n} \rightarrow \mathbb{R}$ such that the leaves of $\mathcal{F}$ are the fibers of $f$. It follows that there is a curve $c_{\mathcal{F}}: I \rightarrow \mathbb{H}^{n} \times \mathbb{R}$ such that the leaves of $\mathcal{F}$ are the umbilical spheres $S_{(t(s), v(s))}$ where $I \subset \mathbb{R}$ and $c_{\mathcal{F}}(s)=(t(s), v(s))$. Since the map $s \rightarrow t(s)$ is $1-1$, we can choose the parameter $s$ so that $t(s)=s$, i.e. we can parametrize $c_{\mathcal{F}}$ by the first factor $t$.

Consider any $C^{1}$ curve $c: I \rightarrow \mathbb{H}^{n} \times \mathbb{R}$ : the umbilical spheres $S_{c(t)}$ may be non-disjoint. We make the following

Claim. The spheres $S_{c(t)}$ are pairwise disjoint if and only if tangent vectors $v^{\prime}(t)$ have hyperbolic norm less than 1 .

We first consider the case $n=1$. Then $v(t)=(\sinh (\eta(t)), \cosh (\eta(t)))$ where $t \rightarrow \eta(t)$ is a $C^{1}$ map. The elements of the 0 -sphere $S_{(t, v(t))}$ are $(\cosh (a), \sinh (a))$ and $(-\cosh (b), \sinh (b))$ where $a, b$ satisfy:

$$
\begin{array}{r}
\cosh (a) \sinh (\eta)-\sinh (a) \cosh (\eta)=\sinh (t) \\
-\cosh (b) \sinh (\eta)-\sinh (b) \cosh (\eta)=\sinh (t)
\end{array}
$$

Hence, we have $a=t-\eta$ and $b=t+\eta$. But the 0 -spheres $S_{c(t)}$ are disjoint if and only if the maps $t \rightarrow a$ and $t \rightarrow b$ are increasing. This is equivalent to the absolute value of $\eta^{\prime}(t)$ being strictly less than 1 . The claim follows since the hyperbolic metric of $\mathbb{H}^{1}$ is $d \eta^{2}$.

Assume now $n \geq 2$. Let $P$ be any 2 -plane in $\mathbb{R}^{1, n}$ on which the restriction of $Q_{1, n}$ has signature $(1,1)$. Let $\pi_{P}: \mathbb{R}^{1, n} \rightarrow P$ be the orthogonal projection. If the $S_{c(t)}$ are two by two disjoint the same is true for the intersections $P \cap S_{c(t)}$, and conversely, if $P \cap S_{c(t)}$ and $P \cap S_{c\left(t^{\prime}\right)}$ are disjoint for every 2-plane as above, then $S_{c(t)}$ and $S_{c\left(t^{\prime}\right)}$ are disjoint. Now observe that the intersection $P \cap S_{c(t)}$ is the set of points $x$ in $P \cap \mathrm{dS}_{n} \approx \mathrm{dS}_{1}$ satisfying $\left\langle x \mid \pi_{P}(v)\right\rangle=\sinh (t)$. Hence, since the $n=1$ case has been 
proved, the spheres $S_{c(t)}$ are all disjoint if and only if for every 2-plane $P$ as above the norm of $d \pi_{P}\left(v^{\prime}(t)\right)$ is less than one. But, using the natural parallelism of $\mathbb{R}^{1, n}$, the spacelike vector $v^{\prime}(t)$ has Minkowski norm less than 1 if and only if all the vectors $d \pi_{P}\left(v^{\prime}(t)\right)=\pi_{P}\left(v^{\prime}(t)\right)$ have Minkowski norm less than 1. The claim follows.

According to the claim, the curve $c_{\mathcal{F}}: I \rightarrow \mathbb{R}$ is a timelike curve in $\mathbb{H}^{n} \times \mathbb{R}$. If this curve is extendible, then it means that some umbilical curve $S_{(T, V)}$ is disjoint from all the $S_{c_{\mathcal{F}}(t)}$. This is a contradiction since $\mathcal{F}$ foliates the entire de Sitter space. Hence, $c_{\mathcal{F}}$ is inextendible.

Conversely, for every inextendible timelike curve $c$ in $\mathbb{H}^{n} \times \mathbb{R}$, the arguments above show that $t \rightarrow S_{c(t)}$ is a 1-parameter family of umbilical spheres which are pairwise disjoint. Since the projection on the second factor of is a Cauchy time function on the globally hyperbolic space $\mathbb{H}^{n} \times \mathbb{R}$, the mean curvature $t$ must take all value in ] $-\infty,+\infty$ [. We leave to the reader the proof that the continuity of $c$ implies that the spheres $S_{c(t)}$ cover all the de Sitter space. It follows that the spheres are the leaves of a CMC-foliation $\mathcal{F}_{c}$.

COROLlaRY 17.6. There are infinitely many non-isometric CMC-foliations of the de Sitter space $d S_{n}$. प

REMARK 17.7 .

1. Proposition 17.5 actually shows that the modulus space of CMC foliations of the de Sitter space $\mathrm{dS}_{n}$ up to isometry is enormous: this is an open set in an infinite dimensional vector space.

2. Proposition 17.5 provides many examples of $\mathrm{CMC}$ foliations of $\mathrm{dS}_{n}$ with poor regularity. Indeed, consider an inextendible timelike curve $c$ in $\mathbb{H}^{n} \times \mathbb{R}$ (equipped with the lorentzian metric $d s_{h y p}^{2}-d t^{2}$ ). The proof of Proposition 17.5 shows how to associate with the curve $c$ a CMC foliation $\mathcal{F}_{c}$ of $\mathrm{dS}_{n}$. Each leaf of the foliation $\mathcal{F}_{c}$ is an umbilical sphere in $\mathrm{dS}_{n}$; in particular, it is an analytic submanifold of $\mathrm{dS}_{n}$. Nevertheless, it follows easily from the construction that the tranverse regularity of the foliation $\mathcal{F}_{c}$ is exactly the same as the regularity of the curve $c$. More precisely, if $\gamma$ is an analytic curve tranverse to the foliation $\mathcal{F}_{c}$, the tangent plane of the leaves of $\mathcal{F}_{c}$ varies in a $C^{k}$ way along $\gamma$ if and only if the curve $c$ is $C^{k}$. Therefore, a curve $c$ which is $C^{k}$ but not $C^{k+1}$ yields a CMC foliation $\mathcal{F}_{c}$ of $\mathrm{dS}_{n}$ which is $C^{k}$ but not $C^{k+1}$.

3. It is well-known that the notion of timelike curve in a lorentz manifold extends to the non-differentiable case: here, it can be defined as curves $c: t \rightarrow \mathbb{H}^{n} \times \mathbb{R}$ such that $c(t)$ is in the strict future of $c\left(t^{\prime}\right)$ for all real numbers $t^{\prime}<t$. Such curves are automatically Lipschitz (see [17]). It is quite obvious that timelike curves in this more general meaning also provide CMC-foliations which are only Lipschitz regular.

4. In Proposition 17.5, we only considered foliations with compact leaves. It is suggestive to relax this condition, i.e. to ask whether CMC-foliations with non compact leaves of $\mathrm{dS}_{n}$ exist and how they behave.

5. The opposite of the mean curvature of an umbilical foliation is a time function. But, not all umbilical time functions are equally "tame". For instance, given any (spacelike compact) hypersurface $S$ in $\mathrm{dS}_{n}$, its isometry group $G_{S}$ (i.e. isometries of $\mathrm{dS}_{n}$ preserving it) has umbilical orbits. The so-obtained time is $G_{S}$-invariant. No other time function can have a "comparable" symmetry group. It is interesting to characterize, variationally, say, these extra- 
symmetric time functions.

17.2.2. Non-trivial quotients of $\mathbf{d} \mathbf{S}_{n}$. In general, an elliptic MGHC de Sitter spacetime is the quotient of $\mathrm{dS}_{n}$ by a finite group $\Gamma$ acting freely on $\mathrm{dS}_{n}$. The group $\Gamma$ admits a fixed point $v_{0}$ in $\mathbb{H}^{n}$. For every real number $t$, the umbilical sphere $S_{\left(t, v_{0}\right)}$ is preserved by $\Gamma$ : it projects in the quotient $M=\Gamma \backslash \mathrm{dS}_{n}$ on a umbilical hypersurface. Hence, varying $t$, we obtain a CMC foliation $\mathcal{F}_{0}$ in $M$. Observe that $M$ admits no CMC time function, since such a CMC time function would lift in $\mathrm{dS}_{n}$ to a CMC time function. Furthermore:

Lemma 17.8. Every compact CMC hypersurface in $M$ is a leaf of $\mathcal{F}_{0}$.

Proof. Let $S$ be a CMC hypersurface in $M$. It lifts to a compact CMC hypersurface in $\mathrm{dS}_{n}$, i.e. to some umbilical sphere $S_{(t, v)}$. It is easy to show that for any isometry $\gamma$ of $\mathrm{dS}_{n}$, either we have $\gamma S_{(t, v)}=S_{(t, v)}$, or there is a transverse intersection between $\gamma S_{(t, v)}$ and $S_{(t, v)}$. Since here $S_{(t, v)}$ is the lift of $S$, the former case cannot occur when $\gamma$ belongs to $\Gamma$. Hence, $v$ must be a fixed point of $\Gamma$. Assume $v \neq v_{0}$. Then, $S_{\left(0, v_{0}\right)}$ is the unit sphere in the euclidean space $v_{0}^{\perp} \approx \mathbb{R}^{n}$, and $v^{\perp} \cap v_{0}^{\perp}$ is a $\Gamma$-hyperplane in this euclidean space. The orthogonal to this hyperplane for the euclidean metric in $v_{0}^{\perp}$ intersects the unit sphere in two points which are both fixed by $\Gamma$ (indeed, these points are fixed individually and not permuted, since one of them belongs to the future of $v^{\perp}$ in $\mathbb{R}^{1, n}$ and the other belongs to the past of $v^{\perp}$ ). This is a contradiction since the action of $\Gamma$ on $\mathrm{dS}_{n}$ is free. Hence, $v=v_{0}$ : the hypersurface $S$ is a leaf of $\mathcal{F}_{0}$.

Corollary 17.6 and Lemma 17.8 conclude the proof of Theorem 1.9.

17.3. The parabolic case. Consider now a parabolic standard spacetime $\mathcal{B}_{0}^{+}(S)$. By definition of parabolic spacetimes, $S$ is the sphere $\mathbb{S}_{+}^{n-1}$ punctured at one point $r_{0}$. The hyperbolic space $\mathbb{H}_{+}^{n}$ is foliated by umbilical hypersurfaces with constant mean curvatures -1 : the horospheres based at $r_{0}$. The dual to these hypersurfaces are umbilical hypersurfaces with the same constant mean curvature -1 , and foliate $\mathcal{B}_{0}^{+}(S)$ (these hypersurfaces are not umbilical spheres, but it is not a contradiction with Montiel's Theorem since they are not compact!). It follows that $\mathcal{B}_{0}^{+}(S)$ admits no CMC time function (since as explained above, if such a CMC time function would exist, then any CMC hypersurface would be a level set of this function; in particular, there would exist at most one CMC hypersurface with mean curvature -1 in $\left.\mathcal{B}_{0}^{+}(S)\right)$.

Every future complete parabolic MGHC dS spacetime is a quotient $M=\Gamma \backslash \mathcal{B}_{0}^{+}(S)$ where $\Gamma$ is a subgroup of $\mathrm{SO}_{0}(1, n)$ preserving $\infty$. As in the previous case, we have a CMC-foliation but no CMC-time. Moreover, let $\Sigma$ be any closed CMC hypersurface. It is tangent to two leaves of the CMC-foliation, one of these leaves being in the future of $\Sigma$, and the other in the past. By the maximum principle, $\Sigma$ has mean curvature -1 ; by the equality case of the maximum principle it follows that $\Sigma$ is equal to the CMC-leaves. In particular, the CMC-foliation is unique. This completes the proof of Proposition 1.10.

REMARK 17.9. Proposition 1.10 also follows directly from [43].

Acknowledgements. The authors are grateful for the hospitality and support of the Isaac Newton Institute in Cambridge, where part of the work on this paper was performed. We thank Ralph Howard for some helpful remarks, and for pointing out reference [26]. 


\section{REFERENCES}

[1] L. Andersson, Constant mean curvature foliations of flat space-times, Commun. Anal. Geom., 10 (2002), pp. 1125-1150.

[2] L. Andersson, The global existence problem in general relativity, The Einstein equations and the large scale behavior of gravitational fields, Birkhäuser, Basel, 2004, pp. 71-120.

[3] _ Constant mean curvature foliations of simplicial spacetimes, Comm. Anal. Geom., 13 (2005), pp. 1-17.

[4] L. Andersson and G. J. Galloway, dS/CFT and spacetime topology, Adv. Theor. Math. Phys., 6:2 (2002), pp. 307-327.

[5] L. Andersson, G. J. Galloway and R. Howard, The Cosmological Time Function, Classical Quantum Gravity, 15 (1998), pp. 309-322.

[6] _ A strong maximum principle for weak solutions of quasi-linear elliptic equations with applications to Lorentzian and Riemannian geometry, Comm. Pure Appl. Math., 51:6 (1998), pp. 581-624.

[7] L. Andersson And R. Howard, Comparison and rigidity theorems in semi-Riemannian geometry, Comm. Anal. Geom., 6:4 (1998), pp. 819-877.

[8] L. Andersson And V. Moncrief, Elliptic-hyperbolic systems and the Einstein equations, Ann. Henri Poincaré, 4:1 (2003), pp. 1-34.

[9] L. Andersson, V. Moncrief And A. J. Tromba, On the global evolution problem in $2+1$ gravity, J. Geom. Phys., 23:3-4 (1997), pp. 191-205.

[10] T. BARbot, Variétés affines radiales de dimension 3, Bull. Soc. math. France, 128 (2000), pp. 347-389.

[11] Flat globally hyperbolic spacetimes, Journ. Geom. Phys., 53 (2005), pp. 123-165.

[12] Causal properties of AdS-isometry groups I: Causal actions and limit sets, Adv. Theor. Math. Phys., 12:1 (2008), pp. 1-66.

[13] T. Barbot, F. BÉGuin, AND A. ZeghiB, Feuilletages des espaces temps globalement hyperboliques par des hypersurfaces à courbure moyenne constante, C. R. Math. Acad. Sci. Paris, 336:3 (2003), pp. 245-250.

[14] - Constant mean curvature foliations of globally hyperbolic spacetimes locally modelled on $A d S_{3}$, Geom. Ded., 126 (2007), pp. 71-129

[15] T. BARbot And A. ZeghiB, Group actions on Lorentz spaces, mathematical aspects: a survey., The Einstein equations and the large scale behavior of gravitational fields, pp. 401-439, Birkhaüser, Basel, 2004.

[16] R. BARTNIK, Remarks on cosmological spacetimes and constant mean curvature surfaces, Comm. Math. Phys., 117:4 (1988), pp. 615-624.

[17] J. K. Beem, P. E. Ehrlich, and K. L. Easley, Global Lorentzian geometry, Monographs and Textbooks in Pure and Applied Mathematics, 2nd ed., 202, Marcel Dekker, New York, 1996.

[18] M. Belraouti, Sur la géométrie de la singularité initiale des espaces-temps plats globalement hyperboliques, arXiv:1201.3716.

[19] R. Benedetti and E. Guadagnini, Cosmological time in $(2+1)$-gravity, Nuclear Phys. B, 613:1-2 (2001), pp. 330-352.

[20] R. Benedetti and F. Bonsante, Canonical Wick rotations in 3-dimensional gravity, Memoirs of the American Mathematical Society, vol. 198, no. 926 -Providence RI (2009).

[21] A. N. Bernal And M. SÁnchez, On smooth Cauchy hypersurfaces and Geroch's splitting theorem, Comm. Math. Phys., 243:3 (2003), pp. 461-470.

[22] - Smoothness of time functions and the metric splitting of globally hyperbolic spacetimes, Comm. Math. Phys., 257:1 (2005), pp. 43-50.

[23] F. Bonsante, Deforming the Minkowskian cone of a closed hyperbolic manifold, Ph. D. Thesis, Pisa, 2005.

[24] F. Bonsante, Flat spacetimes with compact hyperbolic Cauchy surface, Journ. Diff. Geom., 69 (2005), pp. 441-521.

[25] R. Budic, J. Isenberg, L. Lindblom, and P. B. Yasskin, On determination of Cauchy surfaces from intrinsic properties, Comm. Math. Phys., 61:1 (1978), pp. 87-95.

[26] L. A. Caffarelli And X. CABré, Fully nonlinear elliptic equations, American Mathematical Society Colloquium Publications, vol. 43, American Mathematical Society, Providence, RI, 1995.

[27] Y. Choquet-Bruhat and T. Ruggeri, Hyperbolicity of the $3+1$ system of Einstein equations, Comm. Math. Phys., 89:2 (1983), pp. 269-275.

[28] P. T. Chrusciel, J. Isenberg, and D. Pollack, Gluing initial data sets for general relativity, Phys. Rev. Lett., 93 (2004), 081101. 
[29] T. A. Drumm and W. M. Goldman, The geometry of crooked planes, Topology, 38:2 (1999), pp. 323-351.

[30] A. E. Fischer AND V. Moncrief, Conformal volume collapse of 3-manifolds and the reduced Einstein flow, Geometry, mechanics, and dynamics, Springer, New York, 2002, pp. 463522 .

[31] D. Fried, Flat spacetimes, J. Differential Geom., $26: 3$ (1987), pp. 385-396.

[32] C. Gerhardt, H-surfaces in Lorentzian manifolds, Comm. Math. Phys., 89:4 (1983), pp. 523553.

[33] C. Gerhardt, On the CMC foliation of future ends of a spacetime, Pacific J. Math., 226 (2006), pp. 297-308.

[34] C. Godbillon, Feuilletages, études géométriques, Progress in Math., 98 (1991), Birkhäuser.

[35] W. Goldman, Geometric structures on manifolds and varieties of representations, "Geometry of group representations" (Boulder, CO, 1987), pp. 169-198, Contemp. Math., 74, Amer. Math. Soc., Providence, RI, 1988.

[36] S. W. Hawking and G. F. R. Ellis, The large scale structure of space-time, Cambridge University Press, 1973.

[37] K. Krasnov and J.-M. Schlenker, Minimal surfaces and particles in 3-manifolds, Geom. Ded., 126 (2007), pp. 187-254.

[38] R. S. Kulkarni And U. Pinkall, A canonical metric for Möbius structures and its applications, Math. Z., 216:1 (1994), pp. 89-129.

[39] G. A. Margulis, Complete affine locally flat manifolds with a free fundamental group, Zap. Nauchn. Sem. Leningrad. Otdel. Mat. Inst. Steklov. (LOMI), 134 (1984), pp. 190-205, Automorphic functions and number theory, II.

[40] G. Mess, Lorentz Spacetime of Constant Curvature, Geom. Ded., 126 (2007), pp. 3-45.

[41] V. MonCRIEF, Reduction of the Einstein equations in $2+1$ dimensions to a Hamiltonian system over Teichmüller space, J. Math. Phys., 30:12 (1989), pp. 2907-2914.

[42] S. Montiel, An integral inequality for compact spacelike hypersurfaces in de Sitter space and applications to the case of constant mean curvature, Indiana Univ. Math. J., 37 (1988), pp. 909-917.

[43] S. MontiEL, Uniqueness of spacelike hypersurfaces of constant mean curvature in foliated spacetimes, Math. Ann., 314:3 (1999), pp. 529-553.

[44] A. D. Rendall, Theorems on existence and global dynamics for the Einstein equations, Living Rev. Relativ., 5 (2002), 2002-6, 62 pp. (electronic).

[45] K. Scannell, Flat conformal structures and the classification of de Sitter manifolds, Comm. Anal. Geom., 7:2 (1999), pp. 325-345.

[46] A. Treibergs, Entire spacelike hypersurfaces on constant mean curvature in Minkowski space, Invent. Math., 66 (1985), pp. 39-56. 
L. ANDERSSON ET AL. 